\title{
Neuropsychologisch-verhaltenstherapeutisch orientierte Gruppentherapie bei Parkinson-Patienten
}

\author{
Dissertation \\ zur Erlangung der Würde des \\ Doktors der Naturwissenschaften \\ an der Universität Bielefeld
}

Vorgelegt von Jasmin Malak

Bielefeld, 2007 
1. Gutachter

Prof. Dr. Hans J. Markowitsch

2. Gutachter

Prof. Dr. Pasquale Calabrese 
1 Einleitung 5

1.1 Fragestellung der Studie 5

1.2 Entstehung der Arbeit 5

1.3 Studienüberblick 6

2 Theoretischer Hintergrund $\quad 7$

2.1 Vorausgegangene Untersuchungen:

Von den Anfängen des James Parkinson bis in die heutige Zeit $\quad 7$

2.1.1 Aufmerksamkeitsfunktionen:

Mögliche Einschränkungen der Aufmerksamkeitsfähigkeit bei Parkinson-Patienten

2.1.2 Gedächtnisleistungen:

Haben Patienten mit Parkinson-Syndrom tatsächlich defizitären Gedächtnisleistungen?

2.1.3 Exekutive Funktionen:

Beeinträchtigung bei der Ausführung von Aufgaben, die komplexe Denkabläufe erfordern

2.1.4 Psychische Auffälligkeiten:

Welche psychische Veränderungen sind bei Patienten mit Parkinson-Syndrom zu erwarten?

3 Hypothesen

4 Methoden 23

4.1 Studienteilnehmer 23

4.2 Studiendesign 24

4.2.1 Untersuchungs- und Therapieplan 24

4.3 Material und abhängige Variablen 25

4.3.1 Neuropsychologische und psychologische Testinstrumente 25

4.3.1.1 Neuropsychologische Testinstrumente 25

4.3.1.2 Psychologische Testinstrumente 29

4.3.2.3 Neurologische Untersuchung 33 
4.3.2 Neuropsychologische Intervention

4.3.3 Verhaltenstherapeutische Intervention 35

$\begin{array}{ll}\text { 4.3.4 Therapieplan } & 38\end{array}$

$\begin{array}{lll}4.4 & \text { Statistik } & 43\end{array}$

5 Ergebnisse 44

5.1 Aufmerksamkeit und Konzentration $\quad 45$

5.1.1 Aufmerksamkeits- und Konzentrationsleistungen in der 1. Untersuchung

5.1.2 Aufmerksamkeits- und Konzentrationsleistungen in der 2. Untersuchung

5.1.3 Vergleich der Aufmerksamkeitsleistungen in der 1. und 2. Untersuchung

5.2 Gedächtnisleistungen

5.2.1 Gedächtnisleistungen in der 1. Untersuchung

5.2.2 Gedächtnisleistungen in der 2. Untersuchung

5.2.3 Vergleich der Gedächtnisleistungen in der 1. und 2. Untersuchung

5.3 Exekutive Funktionen

5.3.1 Leistungsfähigkeit im Bereich der exekutiven Funktionen in der 1. Untersuchung

5.3.2 Leistungsfähigkeit im Bereich der exekutiven Funktionen in der 2. Untersuchung

5.3.3 Vergleich der Leistungsfähigkeit der exekutiven Funktionen in der 1. und 2. Untersuchung

5.4 Psychische Aspekte

5.4.1 Psychische Aspekte in der 1. Untersuchung

5.4.2 Psychische Aspekte in der 2. Untersuchung

5.4.3 Vergleich der psychischen Aspekte in der 1. und 2. Untersuchung 


\section{Inhalt}

6 Diskussion 93

6.1 Interpretation der Ergebnisse 93

6.1.1 Verbesserung der Aufmerksamkeits- und Konzentrationsleistungen

6.1.2 Steigerung der Gedächtnisleistungen

6.1.3 Verbesserung der exekutiven Funktionen

102

6.1.4 Abnahme depressiver Tendenzen und Ängstlichkeit 105

6.1.5 Zunahme der Zufriedenheit in der Partnerschaft 108

6.2 Allgemeine Diskussion

6.3 Konsequenzen aus der vorliegenden Studie

7 Zusammenfassung

8 Literatur 


\section{Danksagung}

Insbesondere möchte ich mich bei Herrn Prof. Dr. Hans-J. Markowitsch der Universität Bielefeld sehr herzlich dafür bedanken, dass er es mir ermöglicht hat, diese Dissertation schreiben $z u$ können sowie für seine freundliche Unterstützung.

Für die stets inspirierende wissenschaftliche Diskussion und konstruktive Kritik bedanke ich mich ganz besonders bei Herrn Prof. Dr. Pasquale Calabrese, Leiter der Neuropsychologischen Abteilung in der Neurologischen Universitätsklinik Knappschaftskrankenhaus Bochum-Langendreer.

Dr. Bertram Holinka, Oberarzt in der Neurologischen Universitätsklinik Knappschaftskrankenhaus Bochum-Langendreer war bei der Betreuung im Verlauf der praktischen Arbeit stets eine hilfreiche Unterstützung.

Ebenso gebührt mein Dank Herrn PD Dr. Matthias Brand der Universität Bielefeld, der für die Beantwortung von Fragen geduldig zur Verfügung stand.

Auch möchte ich mich bei allen Patienten und ihren Angehörigen bedanken, die an den Untersuchungen und der Therapie teilgenommen haben sowie natürlich bei den beteiligten Kollegen, vor allem Frau Alexandra Häger.

Schließlich möchte ich noch meiner Familie und meinen Freunden danken, vor allem meinem Lebensgefährten Matthias Böttcher sowie meiner Mutter Rita Malak, die mich in „Krisenzeiten“ immer wieder motiviert haben. 


\section{Einleitung}

\subsection{Fragestellung der Studie}

Diese Arbeit soll die Frage nach der Effektivität eines strukturierten neuropsychologischen und verhaltenstherapeutischen Trainingsprogramms zur Verbesserung der allgemeinen kognitiven Leistungen bei Patienten mit Parkinson-Syndrom klären. Darüber hinaus interessiert die Beantwortung der Frage, ob durch eine Einbeziehung der jeweiligen Lebenspartner in die Therapie die Zufriedenheit in der Partnerschaft qualitativ verbessert werden kann.

\subsection{Entstehung der Arbeit}

Im Rahmen meiner Tätigkeit als Neuropsychologin in der Neurologischen Universitätsklinik - Knappschaftskrankenhaus Bochum-Langendreer zeigte sich, dass eine Vielzahl der ambulant bzw. stationär betreuten Parkinson-Patienten (PD-Patienten) mit kognitiven und psychosozialen Schwierigkeiten ein großes Interesse an entsprechenden Therapiemöglichkeiten haben. Die Patienten leiden unter vielschichtigen Problemen, die nicht nur ihre körperlichen Beeinträchtigungen betreffen, sondern über die klassischen Symptome wie Akinese, Rigor und Tremor hinaus auch kognitive und psychosoziale Folgen nach sich ziehen. Das Nachlassen der kognitiven Fähigkeiten bei PD scheint zunächst in erster Linie Aufmerksamkeits-, Konzentrations- und Gedächtnisleistungen sowie Störungen der Exekutivfunktionen zu umfassen. Einige Probleme des psychosozialen Bereichs betreffen z.B. den fehlenden adäquaten Umgang mit der Erkrankung (Krankheitsbewältigung / Coping), den sukzessiven Rückzug aus dem sozialen Leben, Schwierigkeiten in der Partnerschaft oder mangelndes Selbstwertgefühl. 
Auffällig ist, dass die Multidimensionalität der Parkinson-Erkrankung bei ihrer Behandlung kaum ausreichend berücksichtigt wird. Die erheblichen Auswirkungen des Parkinson-Syndroms auf Bewegung, Kognition und Befindlichkeit führen bei Ehepartnern, Familie und dem weiteren sozialen Umfeld der Betroffenen zu einer starken Belastung. Die Hausärzte vor Ort sind häufig überfordert, dem progressiven Fortschreiten der Krankheit und dessen Bewältigung hilfreiche Maßnahmen entgegenzusetzen. Entsprechende Handlungsanweisungen fehlen weitestgehend.

\subsection{Studienüberblick}

In der vorliegenden Arbeit wurden zwei Therapiegruppen, jeweils bestehend aus PD-Patienten und deren Partner, sowie eine Kontrollgruppe, die sich ebenfalls aus PD-Patienten und deren Partner zusammensetzte, untersucht. Die Untersuchungen wurden mit Hilfe neuropsychologischer Testinstrumente sowie anhand von Fragebögen zur Erfassung der Befindlichkeit und psycho-sozialer Aspekte durchgeführt. Die therapeutische Intervention bestand aus computergestützten neuropsychologischen sowie aus verhaltenstherapeutischen Modulen. Die Untersuchungen fanden vor Therapiebeginn und direkt nach Beendigung der Therapie zur Überprüfung des Therapieerfolgs statt. Insgesamt haben 32 Versuchspersonen an der Studie teilgenommen. 


\section{Theoretischer Hintergrund}

\subsection{Vorausgegangene Untersuchungen: Von den Anfängen des James Parkinson bis in die heutige Zeit}

James Parkinson beschrieb im Jahre 1817 in seinem Artikel "An Essay on the Shaking Palsy" die nach ihm benannte Parkinson-Krankheit. Dabei beschrieb er die wichtigsten Symptome, wie die Akinese (Bewegungsarmut/-verlangsamung), den Rigor (Muskelsteifheit) und den Tremor (Zittern). Heute wissen wir, dass es sich um eine neurodegenerative Erkrankung handelt, die durch einen progredienten Verlust von dopaminergen Neuronen in der Substantia nigra zu einem Dopaminmangel in den Basalganglien führt. Daraus resultiert ein Ungleichgewicht der Neurotransmitter mit einer komplexen Störung des Basalganglien-Thalamus-Cortex-Systems, das unterschiedlichste Beeinträchtigungen nach sich ziehen kann (Neher, 1990). Neben den Hauptsymptomen, wie Akinese, Rigor und Tremor, können vegetative und psychische Störungen auftreten. Seit der Einführung von L-Dopa, das den Dopamin-Mangel ausgleicht, gilt die Erkrankung als relativ gut behandelbar, jedoch stellt die abnehmende Medikamentenwirkung mit Fortschreiten der Erkrankung ein großes Problem dar.

Die sozial-medizinische Bedeutsamkeit des Morbus Parkinson zeigt sich allein an ihrer Prävalenz von 1-2 Erkrankten pro 1000 Einwohnern (Scholz \& Oertel, 1993). Da der Gipfel der Häufigkeit der Erkrankung zwischen dem 50. und 70. Lebensjahr liegt, ist aufgrund der Bevölkerungsstruktur in den kommenden Jahren mit einer ansteigenden Tendenz zu rechnen (Schneider, 1994). Männer haben eine etwas höhere Inzidenzrate als Frauen. In 1\%-2\% der Fälle tritt die Krankheit vor dem 40. Lebensjahr auf. Hier spricht man von einer sogenannten „juvenilen“ Parkinson-Erkrankung. Wenn die Ursache der Parkinson-Erkrankung unbekannt ist, dann wird von einem idiopathischen Parkinson-Syndrom gesprochen. Tatsächlich ist die Ursache der Erkrankung nur bei $20 \%$ der Fälle 
bekannt, wie z.B. infektiöser, toxischer, medikamentöser, metabolischer, posttraumatischer, vaskulärer/hypoxischer Ursache. Sie ist selten Teilsyndrom neurodegenerativer Erkrankungen oder durch einen Hirntumor ausgelöst (Scholz \& Oertel, 1993). Im allgemeinen sind Störungen in den Bewegungsabläufen, den vegetativen Funktionen sowie der kognitiven Leistungsfähigkeit und psychischen Befindlichkeit zu beobachten, wobei die letztgenannten von zunehmend größerem Interesse scheinen (z.B. Saint-Cyr, 2003).

Bei der Feststellung einer fraglichen Einschränkung kognitiver Fähigkeiten bei PD-Patienten sollten jedoch unbedingt weitere Faktoren, wie der normale altersbedingte Abbau kognitiver Leistungen, schnelle psychische und physische Ermüdbarkeit, Depressionen, gestörte motorische Fertigkeiten, Beeinflussung durch medikamentöse Nebenwirkungen (z.B. Halluzinationen) sowie Wirkungsschwankungen aufgrund der Einnahme von Medikamenten, sogenannte „On-off-Phänomene“, berücksichtigt werden (Brown \& Marsden, 1987).

Patienten mit Parkinson-Syndrom scheinen im Vergleich zur gesunden Gesamtpopulation, häufiger eine Demenz zu entwickeln (z.B. Hobson \& Meara, 1999). Die Angaben hinsichtlich der Entwicklung einer Demenz bei PD variieren von 9,5\% - 54\% (Aarsland et al., 2001). Aber auch bei PD-Patienten ohne demenzielle Entwicklung können weniger offensichtliche kognitive Beeinträchtigungen beobachtet werden.

Zu Beginn der Erkrankung sind, wenn überhaupt, nur leichte Veränderungen in den kognitiven Leistungen festzustellen. Mit Fortschreiten der Erkrankung kann es jedoch zu deutlichen Defiziten im Bereich der kognitiven Schnelligkeit und Aufmerksamkeit, des Gedächtnisses der visuo-konstruktiven Fähigkeiten sowie der Exekutivfunktionen kommen (z.B. Taylor et al., 1987; Wassenstein et al., 1987; Levin et al., 1991; Bondi et al., 1993; Bublak et al., 2002; Ashby et al., 2003). 


\subsubsection{Aufmerksamkeitsfunktionen: \\ Mögliche Einschränkungen der Aufmerksamkeitsfähigkeit bei Parkinson-Patienten}

Die Aufmerksamkeit mit ihren unterschiedlichen Komponenten ist eine der wichtigsten Leistungen des Gehirns, da die höheren Hirnleistungen auf die Verfügbarkeit der Aufmerksamkeitsfähigkeit angewiesen sind. Die ungerichtete Aufmerksamkeit bezeichnet eine allgemeine Reaktionsbereitschaft und Wachheit (Alertness). Diese ist als basale Aufmerksamkeitsdimension im Sinne einer Aktiviertheit bzw. Aktivierung des Organismus definiert und ist auch die Voraussetzung für "höhere" Aufmerksamkeitsdimensionen, wie der selektiven und der geteilten Aufmerksamkeit. Die Alertness umfaßt zwei Aspekte:

- „Tonisches Arousal“ bezeichnet den physiologischen Wachzustand des Organismus mit einer allgemeinen, zirkadian oszillierenden Aufmerksamkeitsaktivierung. Es handelt sich um das dauernde Aktivierungsniveau, das z.B. von der Tageszeit, vom Ausmaß einer medikamentösen Sedierung usw. abhängt;

- „Phasisches Arousal“ ist die Zunahme der Aufmerksamkeit unmittelbar nach einem Warnreiz (z.B. Warnton), d.h. eine kurzfristige Anhebung des Aufmerksamkeitsniveaus im Sinne einer Orientierungsreaktion, die zu einer Verkürzung der Reaktionszeiten führt (Posner \& Boies, 1971; Posner \& Rafal, 1987).

Im Elektroenzephalogramm (EEG) zeigt sich nach Darbietung eines Warnreizes ein Bereitschaftspotenzial als Zeichen der Aktivierung des Gehirns. Mehrfache Darbietung des Stimulus führt zu Habituation und Abschwächung der Orientierungsreaktion. Es handelt sich um eine komplexe Leistung, die im funktionellen Zusammenwirken verschiedener kortikaler und subkortikaler Hirnstrukturen gesteuert wird. Zahlreiche klinische und experimentelle Studien weisen neben dem Hirnstamm insbesondere der rechten Hirnhälfte eine dominante Rolle bei der Kontrolle der Intensitätsfaktoren der Aufmerksamkeit zu (Howes \& Boller, 1975; Mesulam, 1985; Posner et al., 1987; Ladavas, 1987). 
Eine besondere Bedeutung bei der Aufmerksamkeitsaktivierung hat das noradrenerge System, welches dem Hirnstamm im Locus coeruleus entspringt (z.B. Posner \& Petersen, 1990). Es gibt Hinweise darauf, dass es funktionell stärkere Projektionen vom Locus coeruleus in die rechte Hemisphäre gibt. Hieraus wurde geschlossen, dass es eine kognitiv kontrollierte Regulierung der noradrenergen Aktivierung durch den rechten frontalen Kortex geben muß. Sturm und Kollegen (1999) konnten in einer PET-Studie zeigen, dass ein kortikales und subkortikales, fast ausschließlich rechtshemisphärisches Netzwerk zur Kontrolle und Aufrechterhaltung der Alertness besteht, wobei das anteriore Cingulum und der dorsolaterale frontale Kortex über den Nucleus reticularis die vom noradrenergen System im Hirnstamm bereitgestellte Aufmerksamkeitsaktivierung kontrolliert und kanalisiert.

Nach Bloxham und Mitarbeiter (1987) scheinen Patienten mit ParkinsonSyndrom bei einfachen Reaktionszeitaufgaben langsamere Reaktionszeiten aufzuweisen, als gesunde Kontrollprobanden; sie sind jedoch nach Vorgabe eines Warnreizes in der Lage, ihre Aufmerksamkeit so zu steigern, dass ihre Reaktionszeiten denen der Kontrollpersonen entsprechen. Eine Meta-Analyse klinischer Studien über einfache und komplexe Reaktionszeitaufgaben ergab, dass PD-Patienten im Vergleich zu Kontrollprobanden kein Defizit bei einfachen Reaktionszeitaufgaben zeigen, jedoch bei Reaktionszeitaufgaben, die eine vorherige Entscheidung erfordern, schlechtere Ergebnisse aufweisen (GauntlettGilbert \& Brown, 1998). Jahanshahi und Mitarbeiter (1992) untersuchten 16 PDPatienten und 16 gesunde Kontroll-Probanden, die eine Reaktionszeitaufgabe sowohl mit Warnreiz als auch ohne Warnreiz bearbeiten sollten. PD-Patienten zeigten in der Bedingung ohne Warnreiz gleichschnelle Reaktionszeiten wie die Kontrollpersonen. Auch durch Vorgabe eines Warnreizes, der $200 \mathrm{msec}$ vor dem Stimulus präsentiert wurde, profitierten die PD-Patienten in gleicher Weise wie die Kontrollprobanden. Bei vorheriger $800 \mathrm{msec}$ Darbietung des Warnreizes verkürzten sich die Reaktionszeiten der gesunden Probanden weiterhin, jedoch nicht die der Patienten. Bei einer vorherigen $3200 \mathrm{msec}$ Darbietung des Warnreizes profitierten nun die PD-Patienten wiederum deutlicher, was nach 
Argumentation der Autoren am ehesten durch die langsamere Reaktionsinitiierung der PD-Patienten zu erklären sei.

Zimmermann und Mitarbeiter (1992) gehen davon aus, dass die Resultate hinsichtlich der Reaktionszeiten von PD-Patienten von der Messebene abhängig sind. Nach Auffassung der Autoren konnte in der Mehrzahl der Untersuchungen keine oder allenfalls nur in Teilgruppen eine Verlangsamung der Informationsverarbeitungsgeschwindigkeit bei PD-Patienten gefunden werden.

\subsubsection{Gedächtnisleistungen: \\ Haben Patienten mit Parkinson-Syndrom tatsächlich defizitäre Gedächtnisleistungen?}

Entlang einer chronologischen Achse lassen sich die Gedächtnissysteme unterteilen in einen sensorischen Speicher (Atkinson \& Shiffrin, 1968; Luria, 1973), in den Informationen für einige hundert Millisekunden aufgenommen werden in ein Kurzzeitgedächtnis, das ca. fünf bis neun Items aufnehmen und bis zu einer Minute behalten kann (Miller, 1956) und ein Arbeitsgedächtnis, das für das kurzzeitige Behalten und Manipulieren von Informationen zuständig ist und das z.B. Lernen und schlussfolgerndes Denken erst ermöglicht (Baddeley, 1986, 1990; Shallice, 1992;).

Nach dem Modell von Baddeley und Hitch (1974) entspricht das Arbeitsgedächtnis einem System zur temporären Speicherung und Bearbeitung von Information im Dienste von komplexen kognitiven Prozessen wie Sprachverständnis, Lernen und Problemlösen. „Arbeitsgedächtnis“ bezeichnet demnach ein kognitives System, welches erlaubt, eine begrenzte Menge an Informationen über eine kurze Zeitspanne hinweg zu behalten. Diese Zeitspanne umfasst einige Sekunden. Das Arbeitsgedächtnismodell besteht nach Baddeley (1986) aus den Komponenten der zentralen Exekutive, der phonologischen Schleife und dem visuell-räumlichen Speicher. Die „zentrale Exekutive“ wird als ein generelles Aufmerksamkeitssystem verstanden und dient zur Überwachung, 
Koordination und Kontrolle der beiden "Sklavensysteme" (phonologische Schleife und visuell-räumlicher Speicher). Da die Speicherkapazität limitiert ist, werden automatisierte Speicheraufgaben an die beiden Sklavensysteme delegiert. So werden Kapazitäten für übergeordnete Aufgaben geschaffen. Solche Aufgaben können z.B. das Setzen von Verarbeitungsprioritäten, Unterbrechung von Routineprozessen, Überwachung von nicht-routinierten Prozessen oder Vergleiche von Handlungsergebnissen mit Handlungszielen umfassen. Die zentrale Exekutive verbindet die Sklavensysteme mit dem Langzeitgedächtnis und kann mit dem Supervisory Attentional System (SAS) nch Norman und Shallice (siehe Shallice, 1988) verglichen werden. Die phonologische Schleife speichert temporär für ca. zwei Sekunden auditorische Informationen, es sei denn, diese Informationen werden aktiv durch ein inneres "Rehearsal“ aufrecherhalten. Sie ist vor allem für die Erhaltung von sequentieller Information bzw. für die unmittelbare, serielle Reproduktion verantwortlich. Der visuellräumliche Speicher dient der temporären Erhaltung und Bearbeitung von visuelräumlichen Informationen. Er spielt ebenfalls bei der räumlichen Orientierung und beim visuell-räumlichen Problemlösen eine Rolle. Der visuell-räumliche Speicher verarbeitet visuelle Wahrnehmungen und Vorstellungen und bildet eine Schnittstelle zwischen visuellen und räumlichen Informationen, welche z.T. über das Langzeitgedächtnis abgerufen werden.

Das Langzeitgedächtnis, in das Informationen transferiert und längerfristig gespeichert werden, wird in der Regel in 4 Grundsysteme unterteilt: episodisches Gedächtnis, semantisches Gedächtnis, prozedurales Gedächtnis und Priming. Sowohl das episodische als auch semantische Gedächtnis gehören zum deklarativen Gedächtnis, wobei „deklarativ“ soviel bedeutet wie der intentionale, bewusste und reflektierte Abruf einer Lernsituation, deren Lerninhalt eingeschlossen (Cohen, 1984, 1985; Tulving, 1972, 1983). „Prozedural“ bezieht sich auf Fertigkeiten, die ursprünglich unter bewusstem Lernen erworben wurden, jedoch im Laufe der Zeit automatisiert und unreflektiert ablaufen. 
„Priming“ ist eine gelernte Lernerfahrung bzw. erhöhte Wiedererkennwahrscheinlichkeit nach einer nicht bewusst wahrgenommenen Lernsituation (Cohen \& Squire, 1980; Squire \& Zola-Morgan, 1991; Tulving \& Schachter, 1990). Das Zusammenwirken der vier Systeme basiert nach dem Modell von Tulving (1983) auf der seriellen Einspeicherung, der parallelen Abspeicherung und dem unabhängigen Abruf von Informationen.

Eine zentrale Bedeutung für das Einspeichern neuer Informationen ins Langzeitgedächtnis bzw. deren Konsolidierung wird dem Hippocampus und dem angrenzenden entorhinalen und perirhinalen Cortex, dem anterioren und medialen Thalamus sowie den Strukturen des basalen Vorderhirns zugesprochen. Die medialen temporalen und dienzephalen Strukturen sowie die des basalen Vorderhirns sind in verschiedene miteinander vernetzte Funktionskreise eingebunden, wobei die wichtigsten eine mediale limbische Schleife (der Papezsche Ring) und eine basolaterale limbische Schleife umfassen. Durch die Vermittlung der limbischen Funktionskreise kommt es zur Konsolidierung neuer Information, wobei sie in der Anfangsphase dieses Prozesses für die Rekonstruktion von Gedächtnisinhalten im Rahmen eines bewussten Abrufs zuständig sind (z.B. Gluck \& Myers, 1997). Nach Läsionen des präfrontalen Kortex kann es zu einer Reihe von Gedächtnisauffälligkeiten kommen, wie z.B. Störungen beim freien Abruf, erhöhte Interferenzanfälligkeit sowie Störungen des Gedächtnisses für die zeitliche Abfolge von Ereignissen (Shimamura, 1995).

Studien, die über Beeinträchtigungen des Kurzzeitgedächtnisses und der freien Wiedergabe von Wortlisten berichten zeigen, dass primär Schwierigkeiten bei der Aufnahme und Organisation bzw. der semantischen Einordnung des zu behaltenden Materials, vorliegen könnten. Taylor und Kollegen (1990) stellten in einer Studie fest, dass PD-Patienten größtenteils unauffällige Gedächtnisleistungen aufwiesen, jedoch umschriebene Defizite bei der spontanen Organisation des zu erinnernden Materials und eine gesteigerte 
Sensitivität gegenüber Interferenz beim Lernen hatten. Dies wurde als Hinweis auf ein grundlegendes Defizit bei der Aufnahme neuer Information und deren Enkodierung nach internen Schemata gewertet. Ergebnisse früherer Studien von Brown und Marsden (1987) deuteten auf generalisiert defizitäre visuokonstruktive Fähigkeiten bei PD-Patienten hin. Andere Untersuchungen (z.B. Taylor et al., 1986) ergaben für PD-Patienten hinsichtlich der Aufgabenbearbeitung von visuell-perzeptiven und visuell-räumlichen Tests keine signifikanten Unterschiede im Vergleich zu gesunden Kontrollprobanden. Ransmeyer (1987) führte zwei unterschiedliche visuell-räumliche Tests mit PDPatienten durch: im ersten Test sollten die Probanden abstrakte Figuren in zwei Teile teilen, so dass jeder Teil einem Quadrat entsprach. Hier zeigte sich, dass PD-Patienten in ihren Leistungen gemindert waren. Die zweite Aufgabe beinhaltete Würfelzeichnungen, wobei jeweils 3 Seiten eines Würfels sichtbar waren. Auf jeder Seite war ein unterschiedliches geometrisches Design zu erkennen. Die Probanden sollten nun diesen Würfel mit fünf anderen vergleichen, wobei die korrekte Lösung der rotierten Version des ursprünglichen Würfels entsprach. In der Bearbeitung dieser Aufgabe zeigten die PD-Patienten unauffällige Leistungen. Die Autoren führten die unterschiedlichen Ergebnisse darauf zurück, dass in der letzteren Aufgabe die korrekte Lösung aus einer Auswahl aus Alternativen bestand, während die Lösung der ersten Aufgabe komplett eigenständig gefunden werden mußte.

\subsubsection{Exekutive Funktionen:}

Beeinträchtigungen bei der Ausführung von Aufgaben, die komplexe Denkabläufe erfordern

Nach dem Modell von Shallice (Norman \& Shallice, 1986; Shallice,1982) werden unterschiedliche automatische, unbewusste und aktive Prozesse der Informationsverarbeitung benötigt, um auf die verschiedenen Anforderungen der Umwelt angemessen und zielgerichtet reagieren zu können. Kognition und Handeln basieren auf hoch spezialisierten Programmen. Diese Programme sind hierarchisch geordnet, zielorientiert und für die Kontrolle gelernter Tätigkeiten 
zuständig. Als „eigenständige Wissensmodule“ beinhalten sie die Schemata verschiedener Aspekte unserer Umwelt (Ford, 1988). Ein Schema kann z.B. durch ein anderes Schema oder aber auch durch direkte Umweltstimuli aktiviert werden. Für eine angemessene und zielführende Aktion müssen situationsadäquate Schemata ausgewählt werden. Für diesen Selektionsprozess nehmen Shallice und Norman (1988) zwei qualitativ unterschiedliche Prozesse an: zum einen das "Contention Scheduling“ (CS) und zum anderen das "Supervisory Attentional System“ (SAS). Beide Prozesse (CS, SAS) können unabhängig von einander agieren, aber das SAS ist dem CS übergeordnet und „überwacht“ die Tätigkeiten des CS (Karnath, 1997). Das CS ist für automatische und von außen direkt aktivierte Schemata zuständig. Es reagiert schnell mit geringer mentaler Anstrengung, ist aber in neuen unerwarteten Situationen im Gegensatz zum SAS unflexibel. Das CS bewährt sich vor allem in Routinesituationen. Das SAS ist für komplexere kognitive Problemlösungen zuständig. Es ist dann gefordert, wenn für eine bestimmte Situation keine Schemata zur Verfügung stehen oder aus vorhandenen Schemata neue Prozeduren entwickelt werden müssen (Danek, 2002; Matthes-von Cramon \& Cramon von, 2000; Wallesch, 2002). Das SAS bezeichnet ein generelles und hierarchisch übergeordnetes Planungsprogramm. Es arbeitet mit Schemata aller Hierarchie-Ebenen. Unabhängig von den von außen durch unterschiedlich starke Stimuli aktivierten Schemata, verstärkt das SAS ein schwach aktiviertes oder gehemmtes Schema und kann in den automatischen Auswahlprozess des CS eingreifen. Es können so andere Prioritäten gesetzt und laufende Handlungen modifiziert bzw. unterbrochen werden. Das SAS arbeitet sehr flexibel, ist aber im Vergleich zum CS deutlich langsamer (Norman \& Shallice, 1986; Shallice, 1988).

Der für exekutive Funktionen zuständige Bereich des Gehirns ist der Frontallappen, der sich anterior vor der Fissura centralis befindet. Er wird unterteilt in die motorische Rinde und die davor liegenden prämotorischen und supplementär motorischen Areale sowie das frontale Augenfeld und den präfrontalen Cortex (Brodmann, 1912). Der dorsolaterale Frontallappen ist von Bedeutung für das Ergreifen von Initiativen, Handlungsplanungen, 
Kurzzeitgedächtnisverarbeitung und Supervision (z.B. Fuster, 1997). Der orbitofrontale Bereich ist wichtig für Sozialverhalten, motivationale und emotionale Aspekte, aber auch mitverantwortlich für die Kontrolle autonomer Funktionen (Röhrenbach \& Markowitsch, 1997). Darüber hinaus scheint das linke Frontalhirn für die Aufnahme von Informationen und die rechte Seite für den Abruf von Informationen von Bedeutung zu sein (Fletcher et. al, 1997; Markowitsch, 1995,1997). Nach Schädigung des Frontallappens sind Störungen des Planungsverhaltens eine der häufigsten Folgen (Fuster, 1989; Karnath, 1991).

Die betroffenen Patienten selbst berichten häufig über Schwierigkeiten, Pläne für Aktivitäten entwickeln oder zielgerichtetes Verhalten aufrechterhalten zu können, sowie über Probleme der Konzeptbildung und Überwachung des eigenen Verhaltens (z.B. Brown \& Marsden, 1990; Taylor \& Saint-Cyr, 1995;). Die Patienten können ein unauffälliges Verhalten im Familienkreis zeigen, aber Anpassungsschwierigkeiten in einer ungewohnten Umgebung haben. Dysexekutivfunktionen können als Folge einer erhöhten Anforderung an interne Ressourcen auftreten. Werden diese dann überbeansprucht, ist das Erstellen effizienter Strategien nicht mehr möglich. Insbesondere Patienten mit einer bereits langjährig bestehenden Erkrankung sind hier betroffen (Dubois \& Pillon, 1997; Lees \& Smith, 1983; Owen et al., 1992). Bei der Bearbeitung komplexer Aufgaben haben PD-Patienten aufgrund dieser gestörten Funktionen häufig Schwierigkeiten, irrelevante Informationen zu unterdrücken. Dies kann zu einer übermäßigen kognitiven Belastung bzw. einer verminderten kognitiven Verarbeitungsgeschwindigkeit führen und somit möglicherweise in der Unfähigkeit enden, effiziente Strategien auswählen bzw. ausführen zu können (Brown \& Marsden, 1988; Dalrymple-Alford et al., 1994). Schöttke (1990) berichtet, dass PD-Patienten im Vergleich zu gesunden Kontrollprobanden eine erhöhte Anzahl von Zügen und verlängerte Bearbeitungszeiten sowie andere strategische Verhaltensweisen während der Aufgabenbearbeitung des „Tower of London“ aufweisen. Funke und Grube-Unglaub (1993) beobachteten ebenfalls über verlängerte Lösungszeiten gegenüber nichthirngeschädigten 
Kontrollpatienten bei der Bearbeitung dieser Aufgabe. In einer SPECT-Studie von Müller und Mitarbeitern (2000) wurden 20 PD-Patienten ohne Demenz untersucht. Im Vergleich zu gesunden Kontrollprobanden zeigten die Patienten bei normaler verbaler Intelligenz, gutem Kurzzeitgedächtnis und unauffälliger phasischer Alertness, Defizite im Bereich der exekutiven Funktionen. Die Autoren fanden signifikante Korrelationen zwischen der Dichte der Dopamintransporter (DAT) im Putamen und motorischen Defiziten sowie zwischen DAT im Striatum (Nucleus caudatus und Putamen) und gestörten Frontalhirnfunktionen.

Einige Autoren gehen davon aus, dass ein Vorliegen leicht defizitärer Exekutivfunktionen als prognostischer Hinweis für ein erhöhtes Risiko der Entwicklung einer Demenz bei PD-Patienten gesehen werden kann (z.B. Woods \& Troster, 2003). Andere Autoren wiederum sind der Auffassung, dass Beeinträchtigungen der Exekutivfunktionen bei PD unabhängig von der Entwicklung einer Demenz seien (z.B. Owen et al., 1997; Richards, Cote \& Stern, 1993). Jedoch zeigen sowohl PD-Patienten mit als auch ohne Demenz häufig kognitive Defizite, wie z.B. reduzierte Leistungen des beim Versuch-undIrrtum-Lernen, aber auch bei der Planung von komplexeren Aufgaben. Solche Störungen drücken sich in einer Reihe spezifischer Teilstörungen aus: fehlende Berücksichtigung von Fehlerrückmeldungen (Cicerone et al. 1983; Konow \& Pribram 1970), fehlende Inhibition irrelevanten Verhaltens bei komplexen Handlungsabläufen (Cramon von 1988; Duncan 1986), fehlende Generierung von Subzielen für komplexe Handlungsprobleme (Schöttke 1990; Shallice 1982; Vilkki \& Holst 1989), Fehlen von antizipatorischen Verhaltensweisen (Freedman et al. 1987), häufige Nichtbeachtung von Regeln bzw. fehlende Nutzung von Kontextinformation (Canavan 1983; Schöttke 1993), perseverative Verhaltensweisen (Goldberg \& Bilder 1987) und mangelnde Planung zeitlichsequentiell abhängiger Verhaltensweisen (Canavan et al. 1989; Schöttke 1990). 


\subsubsection{Psychische Auffälligkeiten: \\ Welche psychischen Veränderungen sind bei Patienten mit Parkinson-Syndrom zu erwarten?}

Als häufigste neuro-psychiatrische Komorbidität im Rahmen des ParkinsonSyndroms wird eine Depression diagnostiziert (Tandberg et al.,1996). Die Angaben schwanken zwischen $25 \%$ und $50 \%$ (Gotham et al.,1986). In Abhängigkeit von der Stichprobe, der diagnostischen Instrumente und des Zusammentreffens mit psychiatrischen Problemen variieren die Angaben sogar zwischen $4 \%$ und $70 \%$ (Cummings, 1992). Depressive Zustandsbilder (APA, 1994; Hautzinger et al., 1992) umfassen:

- Somatische Beschwerden (z.B. Schlafstörungen)

- Motorische Probleme (z.B. Verlangsamung der Bewegung)

- Emotionale Beeinträchtigungen (z.B. niedergeschlagene Stimmung)

- Motivationale Störungen (z.B. Interessenverlust)

- Kognitive und sozial-interaktive Beschwerden (z.B. Gedächtnisprobleme, sozialer Rückzug).

Diese Symptome können bei PD-Patienten sowohl durch Störungen der Neurotransmittersysteme verursacht werden als auch reaktiv eine Folge der motorischen oder vegetativen Beeinträchtigungen des Parkinson-Syndroms sein. Der Mangel an Dopamin an sich führt primär zu einem erhöhten Depressionsrisiko (Brown \& Gershon, 1993). Auch wird ein Zusammenhang mit reduzierten Serotonin-Spiegeln im ZNS angenommen (z.B. Mayeux et al., 1984). Als anatomisches Korrelat depressiver Veränderungen wird eine Störung der anterioren cingulären Schleife angenommen (Alexander et al.,1986; Swerdlow \& Koob, 1987).

Depressionen werden häufig als prodromales Symptom des ParkinsonSyndroms angesehen, insbesondere in der Gruppe der PD-Erkrankten unter 40 Jahren sowie bei einem langsamen Verlauf der Erkrankung bzw. bei Patienten, 
bei denen ein Familienmitglied bereits an Parkinson erkrankt ist (z.B. Santamaria et al., 1988). Ein früher Beginn der Erkrankung führt häufig auch zu Schwierigkeiten in der Partnerschaft, wie z.B. zu sexuellen Problemen, oder auch zur Einschränkung sozialer Aktivitäten (z. B. Santamaria et al., 1988; Starkstein et al., 1989). Da die Erstdiagnose meist gestellt wird, wenn bereits ca. $70 \%$ der dopaminergen Zellen zerstört sind, kann kaum Aufschluß darüber erbracht werden, ob und in welcher Richtung ein Zusammenhang zwischen Depression und einem Parkinson-Syndrom besteht. Gotham und Mitarbeiter (1986.) vermuteten persönliche und soziale Ressourcen sowie Einstellung zu Krankheit und Gesundheit als intervenierende Variablen. Zusammenhänge zwischen der Schwere der Erkrankung und Depression sowie zwischen Qualität und Stabilität der Partnerschaft und Depression weisen in diese Richtung (Seiler et al., 1992; Starkstein et al., 1990).

Das Auftreten von Depressionen bei Parkinson-Patienten scheint also abhängig zu sein von der Art und Schwere der strukturellen Beeinträchtigung, dem Ausmaß des Verlusts an Transmittern, der funktionellen Reserve und Plastizität des ZNS sowie den zur Verfügung stehenden persönlichen und sozialen Bewältigungsstrategien (Cummings, 1992). 


\section{Hypothesen}

Die vorliegende Studie überprüft die Effektivität eines strukturierten viermonatigen neuropsychologisch-verhaltenstherapeutisch orientierten Trainingsprogramms bei Patienten mit idiopathischem Parkinson-Syndrom. Mit Hilfe eines in jeder Therapiestunde durchzuführenden, computergestützten Trainings zur Verbesserung kognitiver Leistungen sollen Konzentrations-, Aufmerksamkeits- und Gedächtnisleistungen der teilnehmenden ParkinsonPatienten gesteigert werden. Über die Vermittlung von ausgewählten verhaltenstherapeutischen Strategien sollen positive Veränderungen im Sinne verminderter Depressionsneigung und Ängstlichkeit sowie gesteigerter Zufriedenheit in der Partnerschaft eintreten bzw. Verschlechterungen vermieden werden.

Die Hypothesen lauten im einzelnen:

Hypothese 1

- Es wird eine Verbesserung der Aufmerksamkeits- und Konzentrationsleistungen bei den PD-Patienten sowie bei den gesunden Partnern nach Beendigung der viermonatigen Therapie erwartet.

- Demgegenüber ist von einem Nachlassen der Aufmerksamkeits- und Konzentrationsleistungen der PD-Patienten in der Kontrollgruppe und von konstanten Aufmerksamkeits- und Konzentrationsleistungen bei den gesunden Probanden der Kontrollgruppe nach 4 Monaten auszugehen.

Hypothese 2

- Es sollte eine Steigerung der Gedächtnisleistungen der PD-Patienten und der gesunden Partner nach Beendigung der viermonatigen Therapie verzeichnet werden können. 
- In der Kontrollgruppe sollte bei den PD-Patienten nach 4 Monaten eine Abnahme der Gedächtnisleistungen bzw. gleichbleibende Gedächtnisfunktionen bei den gesunden Probanden beobachtet werden.

Hypothese 3

- Nach Beendigung der viermonatigen Therapie werden verbesserte exekutive Funktionen, sowohl bei den PD-Patienten als auch bei deren gesunder Partnern erwartet.

- Entsprechend ist nach 4 Monaten von einer Verschlechterung der exekutiven Funktionen bei den PD-Patienten der Kontrollgruppe sowie von gleichbleibenden exekutiven Leistungen bei den gesunden Probanden der Kontrollgruppe auszugehen.

Hypothese 4

- Nach Beendigung der viermonatigen Therapie wird eine Abnahme depressiver Tendenzen und Ängstlichkeit bei den PD-Patienten sowie den gesunden Partnern erwartet.

- Nach 4 Monaten wird aufgrund eines weiteren Fortschreitens der Erkrankung sowie auch weiterhin fehlender Coping-Strategien eine Zunahme depressiver Tendenzen und Ängstlichkeit bei den PD-Patienten und den gesunden Partnern in der Kontrollgruppe zu verzeichnen sein.

Hypothese 5

- Schließlich wird von einer Zunahme der Zufriedenheit in der Partnerschaft bei den PD-Patienten sowie bei den gesunden Partnern nach Beendigung der viermonatigen Therapie ausgegangen. 
Bei den PD-Patienten und deren Partnen in der Kontrollgruppe wird nach 4 Monaten die Zufriedenheit in der Partnerschaft abnehmen, da die Patienten aufgrund nachlassender Fähigkeiten, wie z.B. Alltagsaktivitäten selbständig zu bewältigen, eine zunehmende Belastung für ihre Partner darstellen. 


\section{Methoden}

\subsection{Studienteilnehmer}

Die an dieser Studie teilnehmenden Versuchspersonen (VPN) wurden im Rahmen ihrer ambulanten Behandlung im Knappschaftskrankenhaus / Neurologische Universitätsklinik Bochum-Langendreer rekrutiert. Die Teilnahme an dem Studienprojekt war freiwillig und unentgeltlich. Jedem Probanden wurden die Ergebnisse seiner Prä- und Post-Therapieuntersuchungen mitgeteilt. Es nahmen insgesamt 32 Probanden, die sich zur Hälfte aus Patienten mit idiopathischem Parkinson-Syndrom und zur anderen Hälfte aus deren gesunden Lebenspartnern zusammensetzten, an der vorliegenden Studie teil. Alle Teilnehmer mussten folgende Kriterien erfüllen:

- Keine Demenz

- Durchschnittlicher IQ

- Keine Teilnahme an einem weiteren Therapieprojekt

- Keine psychiatrische Erkrankung

- Kein Alkohol-/Drogenmißbrauch

Die 16 VPN, die an einem idiopathischen Parkinson-Syndrom litten, mussten darüber hinaus folgende Krieterien erfüllen:

- Keine weitere neurologische Erkrankung

- Einstufung nach Hoehn und Yahr (1967) in die Stadien 3-5

- Konstante medikamentöse Einstellung

Die Von den 32 Teilnehmern wurden 16 VPN (8 Patienten und deren Partner) der neuropsychologisch-verhaltenstherapeutisch orientierten Gruppentherapie nach dem Zufallsprinzip zugeteilt; die verbleibenden 16 Probanden (ebenfalls 8 Patienten und deren Partner) dienten als Kontrollpersonen. Die Kontrollgruppe 
wurde keiner weiteren therapeutischen Intervention unterzogen und nach 4 Monaten telefonisch kontaktiert, um einen 2. Untersuchungstermin festzulegen. Alle Probanden wurden mündlich über Studiendesign, Testungen und geplantes Therapie-Setting informiert.

\subsection{Studiendesign}

(Neuro-)psychologische Daten wurden in den Therapiegruppen zu 2 Meßzeitpunkten erhoben: vor Beginn der Therapie und am Ende der Therapie. Die strukturierten Therapiesitzungen fanden über 4 Monate wöchentlich mit jeweils einer Doppelstunde (120 Minuten) statt. Die Gruppentherapie-Teilnehmer wurden in 2 Gruppen zu je 8 Personen aufgeteilt (jeweils 4 Patienten und deren gesunde Partner). Auch die 16 Kontrollpersonen wurden zu 2 Meßzeitpunkten untersucht, wobei der Zeitraum zwischen den Untersuchungen ebenfalls 4 Monate betrug.

\subsubsection{Untersuchungs- und Therapieplan}

\begin{tabular}{|c|c|c|c|}
\hline Gruppe & 1. Untersuchung & Therapie & 2. Untersuchung \\
\hline 1. Therapiegruppe & November 2003 & $\begin{array}{r}\text { November 2003 - } \\
\text { Februar 2004 }\end{array}$ & Februar 2004 \\
\hline 2. Therapiegruppe & März 2004 & $\begin{array}{c}\text { März 2004- Juni } \\
2004\end{array}$ & Juni 2004 \\
\hline Kontrollgruppe & April 2004 & -- & Juli 2004 \\
\hline
\end{tabular}




\subsection{Material und abhängige Variablen}

\subsubsection{Neuropsychologische und psychologischeTestinstrumente}

Zur Einschätzung der intellektuellen Leistungsfähigkeit, der Aufmerksamkeit und Konzentration, der Gedächtnisleistungen sowie der Exekutivfunktionen haben sich die VPN einer ausführlichen neuropsychologischen Testbatterie unterzogen. Die zusätzliche Anwendung psychometrischer psychologischer Fragebögen diente dazu, objektive und subjektive Maße miteinander in Beziehung zu setzen, um krankheitsspezifische Probleme und Schwierigkeiten zukünftig besser erkennen und behandeln zu können.

\subsubsection{Neuropsychologische Testinstrumente}

Mehrfachwahl-Wortschatz-Intelligenztest B (MWT-B)

Der Test dient zur Erfassung des verbalen Intelligenzniveaus, wobei er ausschließlich die kristalline Intelligenz messen soll (Lehrl,1989). Testpersonen haben die Aufgabe, bekannte Wörter wiederzuerkennen und diese von unbekannten zu unterscheiden. Der MWT-B soll ein Verfahren sein, das insensitiv gegenüber Störeinflüssen ist, d.h. es soll die Altersstabilität der Testergebnisse gewährleisten (keine Änderung der Ergebnisse ab dem zwanzigsten Lebensjahr) und es ermöglicht die Messung des prämorbiden Intelligenzniveaus bei aktuellen geistigen Leistungsstörungen.

Der MWT-B besteht aus 37 Wortzeilen. In jeder Zeile wird ein umgangs-, wissenschaftlich- oder bildungssprachlich bekanntes Wort aus der deutschen Sprache unter vier sinnlose Wörter gestellt. Die Aufgabe besteht darin, in jeder Zeile das richtige Wort aus der deutschen Sprache herauszufinden und anzustreichen. Jedes angestrichene richtige Wort wird mit einem Punkt bewertet (insgesamt 37 Punkte), wobei Zeilen mit mehr als einem angestrichenen Wort nicht bewertet werden. Der resultierende Rohwert kann in Prozentrang-, Standard- oder IQ-Werte umgewandelt werden. 
Durchführung, Auswertung und Interpretation können als objektiv gelten. Reliabilität: Die Paralleltestkorrelation zwischen MWT-A und MWT-B liegt bei $r=.84$. Untersuchungen zur Retest-Reliabilität ergaben eine Korrelation von $r=.87$ nach 14 Monaten Abstand zwischen den Testzeitpunkten. Zwischen dem MWT$B$ und dem Gesamt-IQ des HAWIE konnte eine Korrelation von $r=.81$ nachgewiesen werden. Die Eichstichprobe bestand aus einer repräsentativen Normstichprobe $(\mathrm{N}=1952)$ für Erwachsene.

Testbatterie zur Aufmerksamkeitsprüfung „Alertness“ (TAP)

Mit dem Subtest „Alertness“ aus der computergestützten Testbatterie zur Aufmerksamkeitsprüfung (TAP) von Zimmermann und Fimm (1993) können tonische und phasische Alertness erfasst werden.

Der Proband hat die Aufgabe, so schnell wie möglich eine Taste zu drücken, sobald in der Mitte des Bildschirms ein Kreuz erscheint. Nach Tastendruck verschwindet das Kreuz und der nächste Durchgang beginnt. Unterschieden werden Durchgänge mit Warnton (phasische Aktivierbarkeit) von Durchgängen ohne Warnton (tonische Aktivierbarkeit). Unmittelbar nach Darbietung des Warnsignals erfolgt der Zielreiz. Es wird angenommen, dass der Proband In den Durchgängen mit Warnton schneller ist, als in den Durchgängen ohne Warnton.

Sowohl Einzelreaktionszeiten, Mittelwert/Median, Standardabweichungen aller Durchgänge, Durchgänge mit bzw. ohne Warnton, Anzahl an Antizipationen und Ausreissern sowie der Kennwert für das Ausmaß der phasischen Alertnessreaktion werden nach Beendigung der Aufgabe aufgelistet. 
Trail-Making-Test-A (TMT-A)

Der Trail-Making-Test Form A, „TMT-A“ (Reitan, 1958) hat die Prüfung der kognitiven Verarbeitungsgeschwindigkeit (Teil A) zum Ziel. Auf einem Blatt sind in randomisierter Anordnung Kreise mit Zahlen von 1-25 gedruckt. Diese sollen möglichst schnell mit einem Bleistift verbunden werden. Die zur Aufgabenbearbeitung benötigte Zeit wird anschließend in altersentsprechende Prozentränge umgewandelt

Trail-Making-Test-B (TMT-B)

Zur Überprüfung der exekutiven Funktionen wurde die kognitive Flexibilität mit dem Trail-Making-Test Form B, „TMT-B“ (Reitan, 1958) untersucht. Hierbei sollen dreizehn Kreise, die die Zahlen von 1-13, und zwölf Kreise, die die Buchstaben von A-L enthalten, in alternierender Reihenfolge (1-A-2-B-3-C usw.) so schnell wie möglich verbunden werden.

Turm von Hanoi (TvH)

Als weitere Leistungen aus dem Bereich der exekutiven Funktionen wurde die Planungs- und Problemlösefähigkeit mit dem Turm von Hanoi getestet, mit dem zeitlich-sequentiell abhängige Handlungsschritte untersucht werden können. Zur Lösung des $\mathrm{TvH}$ muß eine Anzahl unterschiedlich großer Scheiben von einem Anfangsstab auf einen von zwei möglichen Zielstäben gesetzt werden, wobei in einem Zug nur jeweils eine Scheibe bewegt und nie eine größere auf eine kleinere Scheibe gesetzt werden darf. Je nach Anzahl der zu versetzenden Scheiben variiert die Komplexität des Problemraumes exponentiell. Entscheidend für die Lösung ist die Planung sukzessiver Züge. 
Der Proband hat die Aufgabe, nach Erlernen der Zugregeln einen 3er-Turm von desen Ausgangsposition auf den Zielstab zu überführen. Die Dauer der Bearbeitung des TvH hängt von der Problemlösegüte ab. Als abhängiges Maß wird die Anzahl der Züge berechnet, wobei mindestens 7 Züge benötigt werden.

Wechsler Memory Scale-Revised (WMS-R)

Mit der Wechsler Memory Scale Revised, "WMS-R" (Härting et al., 2000; Wechsler, 1987) können die wichtigsten Gedächtnisfunktionen individuell untersucht werden, wobei dem Untersucher Normtabellen und ein allgemeiner Gedächtnisindex zur Verfügung stehen.

Ziel ist im einzelnen die Erfassung der verbalen und nonverbalen Kurzzeit- und Langzeitgedächtnisleistung sowie eines Gedächtnisquotienten, der sich aus der verbalen und visuellen Gedächtnisleistung sowie aus der mittelfristigen Behaltens- und Aufmerksamkeitsleistung zusammensetzt. Die "allgemeine" Gedächtnisleistung wird durch den Gesamtquotienten beschrieben.

Zu den Leistungstests des verbalen Kurzzeit-/ Arbeitsgedächtnisses gehören:

- Logisches Gedächtnis I

- Verbale Paarerkennung I

- Zahlenspanne

Zu den Leistungstests des visuellen Kurzzeit-/Arbeitsgedächtnisses gehören:

- Figurales Gedächtnis

- Visuelle Paarerkennung I

- Visuelle Wiedergabe I

- Blockspanne

Zu den die mittel- bis langfristigen Behaltensleistungen prüfenden Tests gehören: 
- Logisches Gedächtnis II

- Verbale Paarerkennung II

- Visuelle Paarerkennung II

- Visuelle Wiedergabe II

Die Aufmerksamkeitsleistung wird mit dem Untertest „Mentale Kontrolle“ erfasst. Es können Standardwerte für das verbale und visuelle Gedächtnis, für die mittelfristige Behaltensleistung, für die Aufmerksamkeitsleistung und für die allgemeine Gedächtnisleistung berechnet werden (Mittelwert von 100, Standardabweichung von 15). Die Reliabilität liegt im Bereich zwischen . . 4 -.8.

\subsubsection{Psychologische Testinstrumente}

Zur Veränderungsmessung der psychischen Befindlichkeit nach verhaltenstherapeutischer Intervention wurden standardisierte Fragebögen eingesetzt:

Beck Depressions-Inventar (BDI)

Das Beck-Depressions-Inventar (BDI) ist ein Selbstbeurteilungsinstrument zur Erfassung des Schweregrades einer depressiven Symptomatik (Beck \& Steer,1987). Mit diesem Verfahren können klinisch relevante Merkmale für die Zuordnung einer depressiven Erkrankung zu syndromalen Gruppen oder die Veränderung depressiver Beschwerden durch psychotherapeutische bzw. pharmakologische Maßnahmen erhoben werden.

Das BDI entstand unabhängig von einer spezifischen Depressionstheorie aufgrund klinischer Beobachtungen der Beschwerden und Klagen depressiver Patienten. Von depressiven Patienten häufig und von nichtdepressiven Patienten selten berichtete Symptome wurden zu 21 Aussagengruppen zusammengefasst. 
Depression wurde dabei als komplexe Störung aufgefasst, die affektive, kognitive, motivationale, somatische und behaviorale Komponenten umfasst.

Die Items des BDI beziehen sich im Einzelnen auf folgende Symptome:
(A) Traurige Stimmung
(B) Pessimismus
(C) Versagen
(D) Unzufriedenheit
(E) Schuldgefühle
(F) Strafbedürfnis
(G) Selbsthass
(H) Selbstanklagen
(I) Selbstmordimpulse
(J) Weinen
(K) Reizbarkeit
(L) Sozialer Rückzug und Isolierung
(M) Entschlussfähigkeit
(N) Negatives Körperbild
(O) Arbeitsunfähigkeit
(P) Schlafstörungen
(Q) Ermüdbarkeit
(R) Appetitverlust
(S) Gewichtsverlust
(T) Hypochondrie
(U) Libidoverlust

Jede der 21 Symptomgruppen enthält vier Aussagen. Hier sind in einfachen Sätzen die depressiven Symptome in aufsteigendem Schweregrad und zunehmender Beeinträchtigung von $0=$ nicht vorhanden, über $1=$ leichte Ausprägung, 2 = mäßige Ausprägung bis $3=$ starke Ausprägung beschrieben. Der Proband soll nun aus jeder Gruppe die Aussage auswählen, die seine 
gegenwärtige Lage (letzte Woche einschliesslich des aktuellen Tages) am besten beschreibt. Im Testhandbuch (Hautzinger et al., 1995) sind Skalenwerte und Werteverteilung sowie Ergebnisse von Faktorenanalyse, Clusteranalyse und Diskriminanzanalysen zu finden. Zur Auswertung wird ein Summenwert gebildet, der zwischen 0 und 63 Punkten liegt. Werte unter 11 Punkten können grundsätzlich als unauffällig, im normalen Bereich angesehen werden. Werte zwischen 11 und 17 Punkten weisen auf eine milde bis mässige Auspägung depressiver Symptome hin. Als klinisch relevant gilt der Punktwert von 18 und darüber.

Die innere Konsistenz (Cronbach's alpha) erreicht über alle Probanden einen Koeffizienten von .88. Getrennt für die Teilstichproben liegen die Werte zwischen .74 und .92 Der Spearman-Brown Koeffizient liegt bei .84 und der Guttman Koeffizient bei .84. Für die vorliegende deutsche Version des BDI werden Korrelationskoeffizienten mit folgenden Verfahren angegeben: HamiltonDepressionsskala (Hamilton, 1960), Inventar depressiver Symptome (Hautzinger \& Bailer, 1996), SDS, Allgemeine Depressions-Skala (Hautzinger \& Bailer, 1991), Beschwerdenliste (von Zerssen, 1976). Die externe Validität wurde durch die Untersuchung therapiebedingter Veränderungen überprüft.

Fragebogen zur Lebenszufriedenheit (FLZ)

Der Fragebogen zur Lebenszufriedenheit (FLZ) dient der Erfassung relevanter Aspekte der Lebenszufriedenheit in verschiedenen Lebensbereichen (Fahrenberg et al., 2000). Jede der Subskalen umfasst sieben Items, welche auf einer siebenstufigen Antwortskala (von "sehr unzufrieden" bis "sehr zufrieden") beantwortet werden müssen. Die Subskalen messen: Gesundheit, Arbeit und Beruf, finanzielle Lage, Freizeit, Ehe und Partnerschaft, Beziehung zu den eigenen Kindern, Eigene Person, Sexualität, Freunde, Bekannte, Verwandte, Wohnung und die allgemeine Lebenszufriedenheit. 
In der vorliegenden Studie interessierte insbesondere die Messung der "Zufriedenheit in der Partnerschaft". Personen mit einem hohen Wert auf dieser Skala sind mit ihrer Ehe bzw. Partnerschaft hinsichtlich Anforderungen, gemeinsamen Unternehmungen, Offenheit, Verständnis und Hilfsbereitschaft des Partners (der Partnerin) sowie Zärtlichkeit und Geborgenheit zufrieden.

Neben den Normen für die Gesamtstichprobe liegen geschlechtsspezifische und nach sieben Altersgruppen gegliederte Normen vor. Die interne Konsistenz (Cronbachs Alpha) der Skala liegt zwischen .82 und .95. Die Validität ist durch Faktorenanalysen und die Beziehung der Skalen zu anderen Persönlichkeitsmerkmalen gesichert.

Stait-Trait-Anxiety-Inventory (STAI)

Dieses in Forschung und klinischer Praxis eingesetzte Verfahren basiert auf der Unterscheidung von Angst als Zustand und Angst als Eigenschaft. Das TraitModell der Angst, wurde bei der Konzeption des STAI um den Aspekt der Angst als vorübergehenden, emotionalen Zustand, der in seiner Intensität über Zeit und Situation variiert (State-Angst), erweitert. Angst als relativ überdauerndes Persönlichkeitsmerkmal (Trait-Angst, Ängstlichkeit) bezieht sich demgegenüber auf individuelle Unterschiede in der Neigung zu Angstreaktionen. Die zwei Skalen des STAI dienen der Erfassung von Angst als Zustand (State-Angst) und Angst als Eigenschaft (Trait-Angst) und können je nach Fragestellung zusammen oder einzeln eingesetzt werden (Spielberger, 1970).

Die STATE-Angstskala des STAI besteht aus 20 Feststellungen, mit denen der Proband beschreiben soll, wie er sich jetzt, d.h. in diesem Moment fühlt. 10 Feststellungen sind in Richtung Angst formuliert ("Ich bin aufgeregt"; "Ich bin beunruhigt"), 10 andere in Richtung Angstfreiheit ("Ich bin entspannt"; "Ich fühle mich ausgeruht"). Die Beantwortung erfolgt auf einer vierstufigen Skala mit Intensitätsangaben: (1) überhaupt nicht, (2) ein wenig, (3) ziemlich, (4) sehr. Die 
TRAIT-Angstskala des STAI stützt sich ebenfalls auf 20 Feststellungen, mit denen der Proband beschreiben soll, wie er sich im allgemeinen fühlt. 13 Feststellungen sind in Richtung Angst formuliert ("Ich glaube, daß mir meine Schwierigkeiten über den Kopf wachsen"; "Ich mache mir zu viele Gedanken über unwichtige Dinge"), sieben andere in Richtung Angstfreiheit ("Ich fühle mich geborgen"; "Ich bin ausgeglichen"). Die Beantwortung erfolgt auf einer vierstufigen Skala mit Häufigkeitsangaben: (1) fast nie, (2) manchmal, (3) oft, (4) fast immer.

Es liegen T-, Stanine- und Prozentrang-Werte für die Trait-Angst sowie Referenzwerte für studentische und für verschiedene klinische Gruppen vor. Die innere Konsistenz beträgt $r=.90$ für beide Skalen. Die Retestreliabilitäten liegen bei der Trait-Angstskala bei $r=.77$ bis $r=.90$; niedriger liegen die Retestreliabilitäten für die State-Angstskala $(r=.22$ bis $r=.53)$. Die Korrelationen der Trait-Angstskala mit der Skala zur Messung manifester Angst (MAS) von Lück und Timaeus liegen zwischen $r=.73$ und $r=.90$. Die kriterienbezogene Validität wurde durch Korrelation mit anderen Testskalen (u.a. FPI, EPI, EWL, BIV, Paranoid-Depressivitäts-Skala, Beschwerdenliste und verschiedenen subjektiven Streß-Skalen) ermittelt.

\subsubsection{Neurologische Untersuchung}

Die Stadienbestimmung des Parkinson-Syndroms erfolgte nach den Kriterien von Hoehn und Yahr (1967):

Stadium 0: Keine Anzeichen der Erkrankung.

Stadium 1: Einseitige Erkrankung.

Stadium 1.5: Einseitige und axiale Beteiligung.

Stadium 2: Beidseitige Erkrankung ohne Gleichgewichtsstörung.

Stadium 2.5: Leichte beidseitige Erkrankung mit Ausgleich beim Zugtest. 
Stadium 3: Leichte bis mäßige beidseitige Erkrankung; leichte Haltungsinstabilität; körperlich unabhängig.

Stadium 4: Starke Behinderung; kann noch ohne Hilfe laufen oder stehen.

Stadium 5: Ohne Hilfe an den Rollstuhl gefesselt

\subsubsection{Neuropsychologische Intervention}

Computergestütztes Training

Die therapeutische Intervention fand mit Hilfe des neuropsychologischen computergestützten Aufmerksamkeits-, Konzentrations- und Gedächtnistrainings Cogpack 6.0 (Marker,1996) statt. Folgende Subtests kamen zur Anwendung:

"Geschichte“: Trainiert verbale Gedächtnisleistungen, insbesondere das Kurzzeitgedächtnis. Zu historischen Ereignissen sind Jahre zu merken und/oder zu schätzen oder Autoren, Regisseure ihren Werken zuzuordnen. Der Schwierigkeitsgrad wird adaptiv angepasst.

„Route“: Dient zur Verbesserung des visuell-räumlichen Arbeits- und Kurzzeitgedächtnisses. Der Proband muß sich merken, welche Route auf einem formalisierten Stadtplan gefahren wird und diese mit der Maus nachfahren. Der Schwierigkeitsgrad wird adaptiv angepasst.

„Springball“: Dieses Training dient der Aufmerksamkeits- und Reaktionsfähigkeit. Hierbei muß ein Ball auf einem formalisierten, horizontal verschiebbaren Schläger immer wieder hochprallen. Der Schwierigkeitsgrad wird individuell adaptiv angepaßt.

Korrelative Statistiken geben Hinweise auf Alters- und Vorbildungsabhängigkeit. Normtabellen geben Werte für Patienten und Nichtpatienten vor. Statistisch 
signifikante und praktisch relevante Übungserfolge finden sich bereits nach kurzen Übungssequenzen (Marker, 2004).

\subsubsection{Verhaltenstherapeutische Intervention}

Die verhaltenstherapeutische Intervention umfaßte Bausteine aus der Verhaltenstherapie, wie Entspannungsübungen, die Rational-Emotive Therapie, das Selbstinstruktionstraining, die Systematische Desensibilisierung und das Training Sozialer Kompetenz:

\section{Entspannungsübungen}

Als Entspannungsmethode wurde die Kurzversion der Progressiven Muskelentspannung nach Jacobson (Bernstein \& Borkovec, 1975) angewandt. Hierbei sollen die Patienten zunächst ihre Augen schließen und sich auf ihren Atem konzentrieren. Anschließend erfolgt die bewußte Anspannung einzelner Körperpartien, die einige Sekunden gehalten werden soll. Bei der nachfolgenden Entspannung der einzelnen Körperteile sollen nun die Lockerung der Muskeln und das damit zusammenhängende angenehme Gefühl bewusst wahrgenommen werden. Die Patienten sollen lernen, den Unterschied zwischen Anspannung und Entspannung $z u$ erkennen und langfristig die Fähigkeit entwickeln, sich schnell in unterschiedlichen Situationen des Alltags entspannen zu können. Darüber hinaus dient die Entspannungsübung zur Vorbereitung der systematischen Desensibilisierung (siehe weiter unten).

\section{Rational-Emotive Verhaltenstherapie}

Den therapeutischen Kern der Rational-Emotiven Verhaltentherapie (REVT) (Ellis, 1970,1997; Keßler,1982) bildet die Annahme, dass menschliches Denken und menschliche Emotionen eng miteinander verknüpft sind. In der 
therapeutischen Arbeit werden „fehlerhafte“ Gedankenketten identifiziert und analysiert. REVT ist ein multidimensionaler Ansatz, der kognitive, emotive und verhaltensorientierte Techniken einsetzt.

Nach dem ABC-Modell von Ellis sind unangemessene Reaktionen bzw. Krankheitssymptome (consequences) durch die Grundeinstellungen oder Wertsysteme (beliefs) einer Person bestimmt, die durch Erfahrungen oder Ereignisse (anteceding events) ausgelöst werden. Das Ziel der Therapie ist, irrationale Annahmen zu identifizieren und in Frage zu stellen (disputing) und damit unangepassten Reaktionen und Verhaltensweisen den Boden zu entziehen (effect). Ellis listete eine Reihe von irrationalen Grundideen auf, die unrealistisch und übergeneralisierend sind.

\section{Selbstinstruktionstraining}

Das Selbstinstruktionstraining (Meichenbaum, 1997) ist eine Bewältigungstechnik, die an den Selbstverbalisationen des Patienten ansetzt. Ausgehend von der Annahme, dass der innere Monolog bei der Aufrechterhaltung von psychischen Störungen bedeutsam sein kann, werden neue interne Aussagen erarbeitet und eingeübt, welche die Fähigkeit im Umgang mit Problemsituationen verbessern sollen. In der Informationsphase wird der Patient damit vertraut gemacht, wie ungünstige Selbstverbalisationen zu negativen Gefühlen führen und das Problemlösungsverhalten behindern. In der Übungsphase werden positive Selbstverbalisationen erarbeitet, die in der Anwendungsphase in Realsituationen erprobt werden sollen.

\section{Systematische Desensibilisierung}

In der Systematischen Desensibilisierung (Wolpe, 1982), die mit einem Entspannungstraining eingeleitet wird, geht es primär darum, in abgestufter Weise die in möglichst tiefer Entspannung befindlichen Patienten mit 
bedrohlichen Situationen, die plastisch geschildert werden, zu konfrontieren und die normalerweise auftretenden Ängste über den Prozeß der Gegenkonditionierung durch entspannte Reaktionen zu ersetzen.

\section{Training sozialer Kompetenz}

Das Training sozialer Kompetenz dient dem Abbau sozialer Ängste und dem Aufbau angemessener sozialer Fertigkeiten. Es ist ein Verfahren, bei dem verschiedene Techniken zum Einsatz kommen. Deren Auswahl orientiert sich daran, in welchen Bereichen ein Patient spezifischer Förderung bedarf, z.B. bei der Durchsetzung eigener Rechte, beim Ausdrücken eigener Gefühle oder bei der Kontaktaufnahme mit anderen Menschen. Im ersten Teil des Trainings werden die Patienten mit den theoretischen Grundlagen vertraut gemacht: unangemessenes Sozialverhalten ist auf mangelnde soziale Fertigkeiten und ungünstige kognitive Prozesse zurückzuführen. Zugrundegelegt wird ein Modell, das sich aus der Interaktion gefühlsmäßiger, motorischer und kognitiver Faktoren ergibt. Wesentliche Trainingselemente sind Instruktionen und Modellvorgabe, Rollenspiele und Methoden des kognitiven Umstrukturierens (Pfingsten, 1996).

Themenschwerpunkte

Die Therapie umfasste bestimmte Themenschwerpunkte, wie die Verbesserung der Aufmerksamkeits- bzw. Konzentrationsfähigkeit und der Gedächtnisleistungen, Entspannung, Kommunikationsfähigkeit, Steigerung von Selbstsicherheit, Bewältigung von Über- und Unterbeweglichkeit in Stresssituationen und Kommunikation in der Partnerschaft. Die Verteilung der einzelnen Themen über die 16 Therapiestunden ist dem nachfolgenden Therapieplan zu entnehmen: 


\subsubsection{Therapieplan}

1. Stunde:

- Vorstellung des Therapieprogramms

2. Stunde:

- Kennenlernen

- Thema:

„Erwartungen der Teilnehmer an die Therapie“

3. Stunde:

- Thema:

„Vorstellung und Einüben der Entspannungstechnik“

- Hausaufgabe:

○ Tägliches Üben der Entspannungstechnik

4. Stunde:

- Hausaufgabenkontrolle

- Anwendung der Entspannungstechnik

- Thema:

„Aufmerksamkeits- und Gedächtnisstörungen bei Morbus Parkinson “

- Aufmerksamkeits- und Konzentrationstraining am Computer

- Anwendung der Entspannungstechnik

- Hausaufgabe:

○ Entspannungsübungen

5. Stunde:

- Hausaufgabenkontrolle

- Anwendung der Entspannungstechnik

- Thema:

„Einführung in die Systematische Desensibilisierung“

- Anwendung der Entspannungstechnik 
- Hausaufgabe:

- Entspannungsübungen

- Aufstellung von „Angsthierarchien“ individueller Probleme

6. Stunde

- Hausaufgabenkontrolle

- Anwendung der Entspannungstechnik

- Thema:

"Systematische Desensibilisierung: Individuelle Probleme“

- Anwendung der Entspannungstechnik

- Hausaufgabe:

- Entspannungsübungen

- Erarbeiten der Angsthierarchien in-vivo

7. Stunde:

- Hausaufgabenkontrolle

- Anwendung der Entspannungstechnik

- Thema:

„Einführung in die Rational-Emotive Therapie (REVT)“

- Anwendung der Entspannungstechnik

- Hausaufgabe:

- Entspannungsübungen

- Schriftliche Ausarbeitung von Lösungen individueller Probleme anhand der REVT

8. Stunde

- Hausaufgabenkontrolle

- Anwendung der Entspannungstechnik

- Thema:

„Rational-Emotive-Therapie: Gesteigerte Selbstsicherheit“

- Anwendung der Entspannungstechnik 
- Hausaufgabe:

○ Entspannungsübungen

- Schriftliche Ausarbeitung von Lösungen individueller Probleme anhand der REVT

9. Stunde

- Hausaufgabenkontrolle

- Anwendung der Entspannungstechnik

- Thema:

„Einführung in das Selbstinstruktionstraining“

- Anwendung der Entspannungstechnik

- Hausaufgabe:

○ Entspannungsübungen

- Nachbesprechen des Selbstinstruktionstrainings mit dem Partner

10. Stunde

- Hausaufgabenkontrolle

- Anwendung der Entspannungstechnik

- Thema:

„Selbstinstruktionstraining: Über-/Unterbeweglichkeit aufgrund negativer Emotionen und Kognition“

- Computergestütztes Aufmerksamkeits- und Gedächtnistraining

- Anwendung der Entspannungstechnik

- Hausaufgabe:

- Entspannungsübungen

- Anwendung des Selbstinstruktionstrainings in Alltagssituationen

11. Stunde

- Hausaufgabenkontrolle

- Anwendung der Entspannungstechnik

- Thema:

„Einführung in das Kompetenztraining“ 
- Computergestütztes Aufmerksamkeits- und Gedächtnistraining

- Anwendung der Entspannungstechnik

- Hausaufgabe:

○ Entspannungsübungen

- Nachbesprechen des Kompetenztrainings mit dem Partner

12. Stunde

- Hausaufgabenkontrolle

- Anwendung der Entspannungstechnik

- Thema:

„Kompetenztraining: Kommunikation / Kommunikation i.d. Partnerschaft"

- Computergestütztes Aufmerksamkeits- und Gedächtnistraining

- Anwendung der Entspannungstechnik

- Hausaufgabe:

○ Entspannungsübungen

- Anwendung des Kompetenztrainings in Alltagssituationen

13. Stunde

- Hausaufgabenkontrolle

- Anwendung der Entspannungstechnik

- Thema:

„Wiederholung der zuvor besprochenen Therapiemethoden:

Systematische Desensibilisierung, REVT, Selbstinstruktions- und Kompetenztraining"

- Computergestütztes Aufmerksamkeits- und Gedächtnistraining

- Anwendung der Entspannungstechnik

- Hausaufgabe:

○ Entspannungsübungen

- Anwendung der gelernten Therapiemethoden im Alltag

14. Stunde

- Hausaufgabenkontrolle 
- Anwendung der Entspannungstechnik

- Thema:

„Wiederholung der zuvor besprochenen Therapiemethoden:

Systematische Desensibilisierung, REVT, Selbstinstruktions- und Kompetenztraining"

- Computergestütztes Aufmerksamkeits- und Gedächtnistraining

- Anwendung der Entspannungstechnik

- Hausaufgabe:

○ Entspannungsübungen

- Anwendung der gelernten Therapiemethoden im Alltag

15. Stunde

- Hausaufgabenkontrolle

- Anwendung der Entspannungstechnik

- Thema:

„Aktivitätenaufbau“

- Computergestütztes Aufmerksamkeits- und Gedächtnistraining

- Hausaufgabe:

○ Entspannungsübungen

- Aktivitäten gestalten

16.Stunde

- Gemeinsamer Ausflug und Überprüfung der gelernten Techniken in Realsituationen 


\subsection{Statistik}

Die statistische Auswertung wurde mit SPSS 12.0 für Windows durchgeführt. Neben deskriptiven statistischen Verfahren, wie die Berechnung von Häufigkeiten, wurden trotz der relativ geringen Probandenzahl $(n=32)$ auch Verfahren, die eine Normalverteilung voraussetzen, wie der T-Test für gepaarte Stichproben, angewandt. Der Kolmogoroff-Smirnoff-Test auf Normalverteilung wurde nicht durchgeführt, jedoch konnte nach Vorbeurteilung der Daten eine annähernde Normalverteilung angenommen werden.

Die nachfolgend berechneten $p$-Werte sind das Ergebnis statistischer Signifikanztests zur Überprüfung der zuvor formulierten Hypothesen. Die »Nullhypothese« $(\mathrm{H} 0)$ ist die Formulierung der Gleichheit, die »Alternativhypothese« $(\mathrm{H} 1)$ die Formulierung eines Unterschieds bezüglich der interessierenden Fragestellungen. Es wurden gerichtete Hypothesenformulierungen verwendet. Der „p-Wert“ ist die Wahrscheinlichkeit, dass unter der Annahme, die Nullhypothese sei wahr, die Teststatistik den beobachteten oder einen extremeren Wert annimmt. Wenn diese Wahrscheinlichkeit klein ist, so spricht dieses Ergebnis gegen die Nullhypothese und es ist Evidenz für die Richtigkeit der Alternativhypothese vorhanden. Vor der Datenerhebung wurde die maximale Irrtumswahrscheinlichkeit (Signifikanzniveau a) festgelegt. Diese begrenzt den Fehler 1. Art, nämlich die Nullhypothese abzulehnen, obwohl sie richtig ist. Die gewählten Niveaus sind $\alpha=0,05$ und $\alpha=$ 0,01 . Ist der $p$-Wert kleiner als das festgelegte Signifkanzniveau, so ist das Ergebnis „signifikant“ $(p<0,05)$ bzw. „hoch signifikant“ $(p<0,01)$. 


\section{Ergebnisse}

Insgesamt nahmen 32 Probanden (VPN) an der Studie teil. Das Alter der Probanden war über die Therapiebedingungen gleichverteilt. Das durchschnittliche Alter der VPN in der Therapiebedingung betrug 64,68 Jahre, das Alter der VPN in der Kontrollgruppe betrug 65,43 Jahre; (Tab. 1).

Tab.1 Vergleich der Altersmittelwerte aller VPN über die Therapiebedingungen

\begin{tabular}{|c|c|c|c|c|}
\hline Therapiebedingung & $\mathrm{N}$ & $\begin{array}{l}\text { Alter } \\
\text { MW }\end{array}$ & $\begin{array}{l}\text { Alter } \\
\text { SD }\end{array}$ & $\begin{array}{l}\text { Signifikanz } \\
\text { 2-seitig }\end{array}$ \\
\hline Kontrollgruppe & 16 & 65,43 & 5,86 & \multirow{2}{*}{,697 } \\
\hline Therapiegruppen & 16 & 64,68 & 4,88 & \\
\hline
\end{tabular}

$\mathrm{N}=$ Anzahl der Probanden; $\mathrm{MW}=$ Mittelwert; $\mathrm{SD}=$ Standardabweichung

Die zwei Therapiegruppen setzten sich jeweils aus 4 gesunden VPN als auch aus 4 PD-Patienten zusammen. Die Kontrollgruppe bestand aus 16 Teilnehmern (8 gesunde VPN und 8 PD-Patienten); (Tab. 2).

Tab.2 Verteilung der VPN über die Gruppen entsprechend der Diagnosestellung

\begin{tabular}{|l|r|r|r|r|}
\hline \multirow{2}{*}{ Diagnose } & \multicolumn{3}{|c|}{ Gruppe } & \multirow{2}{*}{ Gesamt (N) } \\
\cline { 2 - 5 } & $\begin{array}{c}\text { 1.Therapie- } \\
\text { gruppe (N) }\end{array}$ & $\begin{array}{c}\text { 2. Therapie- } \\
\text { gruppe (N) }\end{array}$ & Kontrollgruppe (N) & 16 \\
\hline Gesunde VPN & 4 & 4 & 8 & 16 \\
\hline PD-Patienten & 4 & 4 & 8 & 32 \\
\hline Gesamt & 8 & 8 & 16 & 16 \\
\hline
\end{tabular}

$\mathrm{N}=$ Anzahl der Probanden; MW=Mittelwert; SD=Standardabweichung

Auch die Geschlechter waren über die Gruppen gleichverteilt: in jeder der 2 Therapiegruppen nahmen 4 Frauen und 4 Männer teil. Die Kontrollgruppe setzte sich aus 8 Frauen und 8 Männer zusammen; (Tab. 3). 
Tab.3 Verteilung der Geschlechter über die Gruppen

\begin{tabular}{|l|r|r|r|r|}
\hline \multirow{2}{*}{ Geschlecht } & \multicolumn{3}{|c|}{ Gruppe } & \multirow{2}{*}{ Gesamt (N) } \\
\cline { 2 - 5 } & $\begin{array}{c}\text { 1. Therapie- } \\
\text { gruppe (N) }\end{array}$ & $\begin{array}{c}\text { 2. Therapie- } \\
\text { gruppe (N) }\end{array}$ & Kontrollgruppe (N) \\
Weiblich & 4 & 4 & 8 & 16 \\
Männlich & 4 & 4 & 8 & 16 \\
\hline Gesamt & 8 & 8 & 16 & 32 \\
\hline
\end{tabular}

$\mathrm{N}=$ Anzahl der Probanden; MW=Mittelwert; $\mathrm{SD}=$ Standardabweichung

Das verbale Intelligenzniveau (gemessen mit dem MWT-B) war bei den Teilnehmern annähernd gleich hoch, wobei die Teilnehmer in den Therapiegruppen einen Mittelwert (MW) von 28,87 Punkten (IQ=104), SD=2,55 Punkte und die der Kontrollgruppe $M W=28,37$ Punkte $(I Q=103), S D=2,91$ Punkte, erreichten ( $p=0.61)$; (Tab. 4).

Tab.4 Vergleich der Intelligenzniveau-Mittelwerte über die Gruppen, gemessen mit dem MWT-B

\begin{tabular}{|l|r|r|r|r|}
\hline Therapiebedingung & N & MWT-B & MW & SD \\
\hline Kontrollgruppe & 16 & 28,37 & 2,55 & $\begin{array}{c}\text { Signifikanz } \\
\text { 2-seitig }\end{array}$ \\
Therapiegruppen & 16 & 28,87 & 2,91 &, 610 \\
\hline
\end{tabular}

$\mathrm{N}=$ Anzahl der Probanden; MW=Mittelwert; SD=Standardabweichung

\subsection{Aufmerksamkeit und Konzentration}

\subsubsection{Aufmerksamkeits- und Konzentrationsleistungen in der 1. Untersuchung}

In der 1. neuropsychologischen Untersuchung zeigten die PD-Patienten der Therapiegruppen im Vergleich zu den PD-Patienten der Kontrollgruppe im Hinblick auf die basale Aufmerksamkeitsfähigkeit, gemessen mit der Testbatterie zur Aufmerksamkeitsprüfung (TAP) „Alertness ohne Warnton“ keine signifikanten Unterschiede. Die Steigerungsfähigkeit der Aufmerksamkeit, gemessen mit der TAP „Alertness mit Warnton“ sowie die kognitive Schnelligkeit, gemessen mit dem Trail-Making-Test-A (TMT-A) war in beiden Gruppen ebenfalls als nicht signifikant unterschiedlich $\mathrm{zu}$ bewerten. Auch die Überprüfung der Aufmerksamkeits- und Konzentrationsfähigkeit anhand der Punktwerte des 
entsprechenden Subtests der Wechsler-Memory-Scale-R ergab keinen signifikanten Unterschied in den durchschnittlich erreichten Mittelwerten zwischen den Gruppen; (Tab. 5).

Tab.5 Mittelwerte der Aufmerksamkeitsleistungen PD-Patienten in Kontroll- und Therapiebedingung in der 1.neuropsychologischen Untersuchung, gemessen mit TAP-Alertness und WMS-R-Subtest „Aufmerksamkeit und Konzentration“

\begin{tabular}{|c|c|c|c|c|c|}
\hline $\begin{array}{l}\text { 1. neuropsychologische } \\
\text { Untersuchung } \\
\text { Testverfahren }\end{array}$ & PD-Patienten & $\mathrm{N}$ & MW & SD & $\begin{array}{l}\text { Signifikanz } \\
\text { 2-seitig }\end{array}$ \\
\hline $\begin{array}{l}\text { Alertness } \\
\text { ohne Warnton (TAP) }\end{array}$ & $\begin{array}{l}\text { Kontrollgruppe } \\
\text { Therapiegruppen }\end{array}$ & $\begin{array}{l}8 \\
8\end{array}$ & $\begin{array}{l}269,37 \mathrm{msec} \\
318,33 \mathrm{msec}\end{array}$ & $\begin{array}{l}22,48 \mathrm{msec} \\
52,35 \mathrm{msec}\end{array}$ & ,027, \\
\hline $\begin{array}{l}\text { Alertness } \\
\text { mit Warnton (TAP) }\end{array}$ & $\begin{array}{l}\text { Kontrollgruppe } \\
\text { Therapiegruppen }\end{array}$ & $\begin{array}{l}8 \\
8\end{array}$ & $\begin{array}{l}249,25 \mathrm{msec} \\
287,11 \mathrm{msec}\end{array}$ & $\begin{array}{l}26,87 \mathrm{msec} \\
54,66 \mathrm{msec}\end{array}$ & 097, \\
\hline $\begin{array}{l}\text { Trail-Making-Test-A } \\
\text { (TMT-A) }\end{array}$ & $\begin{array}{l}\text { Kontrollgruppe } \\
\text { Therapiegruppen }\end{array}$ & $\begin{array}{l}8 \\
8\end{array}$ & $\begin{array}{l}65,12 \mathrm{sec} \\
64,66 \mathrm{sec}\end{array}$ & $\begin{array}{l}21,35 \mathrm{sec} \\
23,62 \mathrm{sec}\end{array}$ & 967, \\
\hline $\begin{array}{l}\text { Aufmerksamkeit, } \\
\text { Konzentration } \\
\text { (WMS-R) }\end{array}$ & $\begin{array}{l}\text { Kontrollgruppe } \\
\text { Therapiegruppen }\end{array}$ & $\begin{array}{l}8 \\
8\end{array}$ & $\begin{array}{r}101,12 \\
95,44\end{array}$ & $\begin{array}{l}15,16 \\
11,71\end{array}$ & 398, \\
\hline
\end{tabular}

$\mathrm{N}=$ Anzahl der Probanden; $\mathrm{MW}=$ Mittelwert; $\mathrm{SD}=$ Standardabweichung; $\mathrm{msec}=$ Millisekunden; $\mathrm{sec}=$ Sekunden;

Zwischen den gesunden VPN der Therapiegruppen und der Kontrollgruppe fand sich in der 1. neuropsychologischen Untersuchung hinsichtlich der basalen Aufmerksamkeit „Alertness ohne Warnton“, der Steigerungsfähigkeit der Aufmerksamkeit, gemessen mit TAP „Alertness mit Warnton“, der kognitiven Schnelligkeit (TMT-A) oder in den Konzentrationsleistungen, gemessen mit dem Subtest „Konzentration“ aus der WMS-R kein signifikanter Unterschied; (Tab. 6).

Tab.6 Mittelwerte der Aufmerksamkeitsleistungen der gesunden VPN in Kontroll- und Therapiebedingng in der 1. neuropsychologischen Untersuchung, gemessen mit TAP-Alertness und WMS-R-Subtest „Aufmerksamkeit und Konzentration"

\begin{tabular}{|c|c|c|c|c|c|}
\hline $\begin{array}{c}\text { 1.neuropsychologische } \\
\text { Untersuchung } \\
\text { Testverfahren }\end{array}$ & Gesunde VPN & $\mathrm{N}$ & Mittelwert & SD & $\begin{array}{l}\text { Signifikanz } \\
\text { 2-seitig }\end{array}$ \\
\hline \multirow{2}{*}{$\begin{array}{l}\text { Alertness } \\
\text { ohne Warnton (TAP) }\end{array}$} & Kontrollgruppe & 8 & $245,50 \mathrm{msec}$ & $33,42 \mathrm{msec}$ & \multirow{2}{*}{,360 } \\
\hline & Therapiegruppen & 8 & $259,57 \mathrm{msec}$ & $21,77 \mathrm{msec}$ & \\
\hline \multirow{2}{*}{$\begin{array}{l}\text { Alertness } \\
\text { mit Warnton (TAP) }\end{array}$} & Kontrollgruppe & 8 & $231,87 \mathrm{msec}$ & $36,11 \mathrm{msec}$ & \multirow{2}{*}{,441 } \\
\hline & Therapiegruppen & 8 & $246,57 \mathrm{msec}$ & $35,20 \mathrm{msec}$ & \\
\hline \multirow{2}{*}{$\begin{array}{l}\text { Trail-Making-Test-A } \\
\text { (TMT-A) }\end{array}$} & Kontrollgruppe & 8 & $49,37 \mathrm{sec}$ & $15,72 \mathrm{sec}$ & \multirow{2}{*}{,778 } \\
\hline & Therapiegruppen & 8 & $47,00 \mathrm{sec}$ & $16,23 \mathrm{sec}$ & \\
\hline \multirow{2}{*}{$\begin{array}{l}\text { Aufmerksamkeit, } \\
\text { Konzentration (WMS-R) }\end{array}$} & Kontrollgruppe & 8 & 98,25 & 13,34 & \multirow{2}{*}{, 067} \\
\hline & Therapiegruppen & 8 & 85,14 & 11,83 & \\
\hline
\end{tabular}

$\mathrm{N}=$ Anzahl der Probanden; MW=Mittelwert; $\mathrm{SD}=$ Standardabweichung ; $\mathrm{msec}=$ Millisekunden; $\mathrm{sec}=$ Sekunden 
Im Vergleich zu den PD-Patienten waren die gesunden Probanden der Therapiebedingung in der 1. neuropsychologischen Untersuchung signifikant besser in der basalen Aufmerksamkeit „Alertness ohne Warnton“, wobei die PDPatienten der Therapiegruppen einen Mittelwert (MW) von 318,33 msec, $\mathrm{SD}=52,35 \mathrm{msec}$ und die gesunden VPN der Therapiegruppen einen MW=259,57 msec, $S D=21,77$ msec $(p<0.05)$ erreichten; (Abb. 1; Tab. 7).

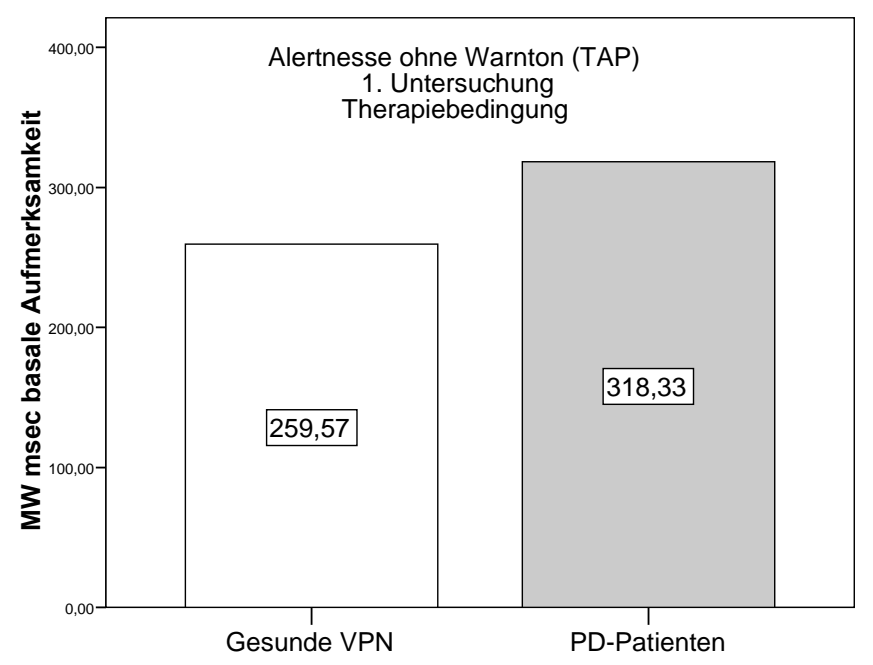

Abb.1 Basale Aufmerksamkeitsleistungen der gesunden VPN und
PD-Patienten in Therapiebedingung in der 1. Untersuchung $(p=0,015)$

Weitere signifikante Unterschiede zwischen den gesunden VPN und den PDPatienten der Therapiegruppen konnten bzgl. der Aufmerksamkeits- und Konzentrationsleistungen nicht beobachtet werden (Tab. 7). 
Tab.7 Mittelwerte der Aufmerksamkeitsleistungen der PD-Patienten und der gesunden VPN in Therapiebedingung in der 1. neuropsychologischen Untersuchung, gemessen mit TAP-Alertness, TMT-A und WMS-R-Subtest „Aufmerksamkeit und Konzentration“

\begin{tabular}{|c|c|c|c|c|c|}
\hline $\begin{array}{c}\text { 1.neuropsychologische } \\
\text { Untersuchung } \\
\text { Testverfahren }\end{array}$ & $\begin{array}{c}\text { VPN } \\
\text { Therapiegruppen }\end{array}$ & $\mathrm{N}$ & MW & SD & $\begin{array}{c}\text { Signifikanz } \\
\text { 2-seitig }\end{array}$ \\
\hline $\begin{array}{l}\text { Alertness } \\
\text { ohne Warnton (TAP) }\end{array}$ & $\begin{array}{l}\text { Gesunde VPN } \\
\text { PD-Patienten }\end{array}$ & $\begin{array}{l}8 \\
8\end{array}$ & $\begin{array}{l}259,57 \mathrm{msec} \\
318,33 \mathrm{msec}\end{array}$ & $\begin{array}{l}21,77 \mathrm{msec} \\
52,35 \mathrm{msec}\end{array}$ & ,015 \\
\hline $\begin{array}{l}\text { Alertness } \\
\text { mit Warnton (TAP) }\end{array}$ & $\begin{array}{l}\text { Gesunde VPN } \\
\text { PD-Patienten }\end{array}$ & $\begin{array}{l}8 \\
8\end{array}$ & $\begin{array}{l}246,57 \mathrm{msec} \\
287,11 \mathrm{msec}\end{array}$ & $\begin{array}{l}35,20 \mathrm{msec} \\
54,66 \mathrm{msec}\end{array}$ &, 111 \\
\hline $\begin{array}{l}\text { Trail-Making-Test-A } \\
\text { (TMT-A) }\end{array}$ & $\begin{array}{l}\text { Gesunde VPN } \\
\text { PD-Patienten }\end{array}$ & $\begin{array}{l}8 \\
8\end{array}$ & $\begin{array}{l}47,00 \mathrm{sec} \\
64,66 \mathrm{sec}\end{array}$ & $\begin{array}{l}16,23 \mathrm{sec} \\
23,62 \mathrm{sec}\end{array}$ & ,114 \\
\hline $\begin{array}{l}\text { Aufmerksamkei, } \\
\text { Konzentration (WMS-R) }\end{array}$ & $\begin{array}{l}\text { Gesunde VPN } \\
\text { PD-Patienten }\end{array}$ & $\begin{array}{l}8 \\
8\end{array}$ & $\begin{array}{l}85,14 \\
95,44\end{array}$ & $\begin{array}{l}11,83 \\
11,71\end{array}$ & ,104 \\
\hline
\end{tabular}

$\mathrm{N}=$ Anzahl der Probanden; MW=Mittelwert; SD=Standardabweichung ; msec= Millisekunden; sec=Sekunden

Im Vergleich zu den PD-Patienten der Kontrollgruppen konnten die gesunden Probanden der Kontrollgruppen in der 1. neuropsychologischen Untersuchung keine signifikant besseren Leistungen in der basalen Aufmerksamkeit „Alertness ohne Warnton“, in der Steigerungsfähigkeit der Aufmerksamkeit, gemessen mit TAP „Alertness mit Warnton“ in der kognitiven Schnelligkeit (TMT-A) in der Konzentration, gemessen mit dem Subtest „Konzentration“ aus der WMS-R, erzielen ; (Tab. 8).

Tab.8 Mittelwerte der Aufmerksamkeitsleistungen der PD-Patienten und der gesunden VPN in Kontrollbedingung in der 1. neuropsychologischen Untersuchung, gemessen mit TAP-Alertness, TMT-A und WMS-R-Subtest „Aufmerksamkeit und Konzentration“

\begin{tabular}{|l|l|r|r|r|r|}
\hline $\begin{array}{c}\text { 1.neuropsychologische } \\
\text { Untersuchung } \\
\text { Testverfahren }\end{array}$ & \multicolumn{1}{c|}{$\begin{array}{c}\text { VPN } \\
\text { Kontrollgruppe }\end{array}$} & N & MW & SD & $\begin{array}{c}\text { Signifikanz } \\
\text { 2-seitig }\end{array}$ \\
\hline $\begin{array}{l}\text { Alertness } \\
\text { ohne Warnton TAP }\end{array}$ & Gesunde VPN & 8 & $245,50 \mathrm{msec}$ & $33,42 \mathrm{msec}$ &, 116 \\
\hline Alertness & PD-Patienten & 8 & $269,37 \mathrm{msec}$ & $22,48 \mathrm{msec}$ &, 293 \\
mit Warnton (TAP) & Gesunde VPN & 8 & $231,87 \mathrm{msec}$ & $36,11 \mathrm{msec}$ &, 115 \\
\hline Trail-Making-Test & PD-Patienten & 8 & $249,25 \mathrm{msec}$ & $26,87 \mathrm{msec}$ & \\
(TMT-A) & Gesunde VPN & 8 & $49,37 \mathrm{sec}$ & $15,72 \mathrm{sec}$ &, 693 \\
\hline Aufmerksamkeit, & PD-Patienten & 8 & $65,12 \mathrm{sec}$ & $21,35 \mathrm{sec}$ & \\
Konzentration (WMS-R) & PD-Patienten & 8 & 98,25 & 13,34 & \\
& PDinde VPN & 8 & 101,12 & 15,16 & \\
\hline
\end{tabular}

$\mathrm{N}=$ Anzahl der Probanden; MW=Mittelwert; SD=Standardabweichung ; $\mathrm{msec}=$ Millisekunden; $\mathrm{sec}=$ Sekunden 


\subsubsection{Aufmerksamkeits- und Konzentrationsleistungen in der 2. Untersuchung}

Nach Beendigung der Therapie zeigten die PD-Patienten der Therapiegruppen im Vergleich zur 2. Untersuchung der Patienten der Kontrollgruppe tendenzielle, jedoch nicht signifikante, Verbesserungen in der basalen Aufmerksamkeit "Alertness ohne Warnton“ (TAP). Die Steigerungsfähigkeit der Aufmerksamkeit, TAP „Alertness mit Warnton“, war in beiden Gruppen auch weiterhin als nicht signifikant unterschiedlich zu bewerten. Die Überprüfung der kognitiven Schnelligkeit mit dem TMT-A in der 2. Untersuchung zeigte wiederum eine tendenzielle, nicht signifikante Verbesserung in den Therapiegruppen; (Tab. 9).

Auch die anhand der WMS-R gemessene Aufmerksamkeits- und Konzentrationsleistung nach Beendigung der Therapie ergab für die Therapiegruppen einen tendenziell, jedoch nicht signifikant verbesserten Wert; (Tab. 9).

Tab.9 Mittelwerte der Aufmerksamkeitsleistungen der PD-Patienten in Kontroll- und Therapiebedingung in der 2 neuropsychologischen Untersuchung gemessen mit TAP-Alertness,TMT-A und WMS-R-Subtestest „Aufmerksamkeit und Konzentration“

\begin{tabular}{|c|c|c|c|c|c|}
\hline $\begin{array}{l}\text { 2. neuropsychologische } \\
\text { Untersuchung } \\
\text { Testverfahren }\end{array}$ & PD-Patienten & $\mathrm{N}$ & MW & SD & $\begin{array}{c}\text { Signifikanz } \\
\text { 2-seitig }\end{array}$ \\
\hline $\begin{array}{l}\text { Alertness } \\
\text { ohne Warnton (TAP) }\end{array}$ & $\begin{array}{l}\text { Kontrollgruppe } \\
\text { Therapiegruppen }\end{array}$ & $\begin{array}{l}8 \\
8\end{array}$ & $\begin{array}{l}296,62 \mathrm{msec} \\
269,22 \mathrm{msec}\end{array}$ & $\begin{array}{l}31,89 \mathrm{msec} \\
57,11 \mathrm{msec}\end{array}$ & ,249 \\
\hline $\begin{array}{l}\text { Alertness } \\
\text { Mit Warnton (TAP) }\end{array}$ & $\begin{array}{l}\text { Kontrollgruppe } \\
\text { Therapiegruppen }\end{array}$ & $\begin{array}{l}8 \\
8\end{array}$ & $\begin{array}{l}249,55 \mathrm{msec} \\
249,50 \mathrm{msec}\end{array}$ & $\begin{array}{l}32,78 \mathrm{msec} \\
51,59 \mathrm{msec}\end{array}$ & ,998 \\
\hline $\begin{array}{l}\text { Trail-Making-Test-A } \\
\text { (TMT-A) }\end{array}$ & $\begin{array}{l}\text { Kontrollgruppe } \\
\text { Therapiegruppen }\end{array}$ & $\begin{array}{l}8 \\
8\end{array}$ & $\begin{array}{l}56,00 \mathrm{sec} \\
53,25 \mathrm{sec}\end{array}$ & $\begin{array}{l}20,08 \mathrm{sec} \\
30,65 \mathrm{sec}\end{array}$ & ,832 \\
\hline $\begin{array}{l}\text { Aufmerksamkeit, } \\
\text { Konzentration (WMS-R) }\end{array}$ & $\begin{array}{l}\text { Kontrollgruppe } \\
\text { Therapiegruppen }\end{array}$ & $\begin{array}{l}8 \\
8\end{array}$ & $\begin{array}{r}89,62 \\
105,55\end{array}$ & $\begin{array}{l}13,07 \\
20,54\end{array}$ & ,080 \\
\hline
\end{tabular}

$\mathrm{N}=$ Anzahl der Probanden; MW=Mittelwert; SD=Standardabweichung; $\mathrm{msec}=$ Millisekunden; sec=Sekunden

Die gesunden VPN beider Gruppen unterschieden sich in der 2. neuropsychologischen Untersuchung nicht signifikant voneinander hinsichtlich der basalen Aufmerksamkeit "Alertness ohne Warnton“, in der Steigerungsfähigkeit der Aufmerksamkeit, gemessen mit TAP „Alertness mit 
Warnton“ und in der kognitiven Schnelligkeit (TMT-A). Die Konzentrationsfähigkeit, gemessen mit dem Subtest „Aufmerksamkeit und Konzentration“ aus der WMS-R konnte bei den gesunden VPN der Therapiegruppen mit einem deutlich höheren Wert von 108,14 Punkten, SD= 11,62 Punkte; Kontrollgruppe mit 94,75Punkten, SD=9,28 Punkte signifikant verbessert werden $(p<0.05)$; (Abb. 2; Tab. 10)

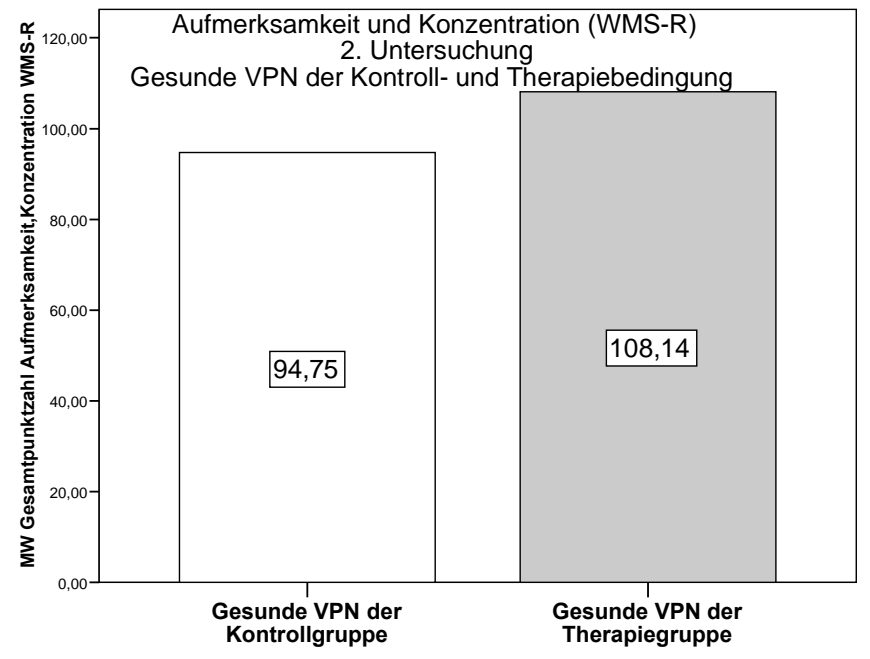

Abb.2 Aufmerksamkeits- und Konzentrationsleistung der gesunden VPN der Kontroll- und Therapiebedingungen in der 2. Untersuchung $(p=0,028)$

Tab.10 Mittelwerte der Aufmerksamkeitsleistungen der gesunden VPN in Kontroll- und Therapiebedingung in der 2.neuropsychologischen Untersuchung, gemessen mit TAP-Alertness, TMT-A und WMS-R-Subtest „Aufmerksamkeit und Konzentration“

\begin{tabular}{|c|c|c|c|c|c|}
\hline $\begin{array}{l}\text { 2.neuropsychologische } \\
\text { Untersuchung } \\
\text { Testverfahren }\end{array}$ & Gesunde VPN & $\mathrm{N}$ & Mittelwert & SD & $\begin{array}{l}\text { Signifikanz } \\
\text { 2-seitig }\end{array}$ \\
\hline $\begin{array}{l}\text { Alertness } \\
\text { ohne Warnton (TAP) }\end{array}$ & $\begin{array}{l}\text { Kontrollgruppe } \\
\text { Therapiegruppen }\end{array}$ & $\begin{array}{l}8 \\
8\end{array}$ & $\begin{array}{l}252,00 \mathrm{msec} \\
248,71 \mathrm{msec}\end{array}$ & $\begin{array}{l}34,92 \mathrm{msec} \\
22,35 \mathrm{msec}\end{array}$ & ,835 \\
\hline $\begin{array}{l}\text { Alertness } \\
\text { mit Warnton (TAP) }\end{array}$ & $\begin{array}{l}\text { Kontrollgruppe } \\
\text { Therapiegruppen }\end{array}$ & 8 & $\begin{array}{l}234,50 \mathrm{msec} \\
232,00 \mathrm{msec}\end{array}$ & $\begin{array}{l}38,39 \mathrm{msec} \\
26,88 \mathrm{msec}\end{array}$ & ,888 \\
\hline $\begin{array}{l}\text { Trail-Making-Test-A } \\
\text { (TMT-A) }\end{array}$ & $\begin{array}{l}\text { Kontrollgruppe } \\
\text { Therapiegruppen }\end{array}$ & 8 & $\begin{array}{l}43,62 \mathrm{sec} \\
36,42 \mathrm{sec}\end{array}$ & $\begin{array}{r}13,19 \mathrm{sec} \\
8,07 \mathrm{sec}\end{array}$ & ,233 \\
\hline $\begin{array}{l}\text { Aufmerksamkeit, } \\
\text { Konzentration (WMS-R) }\end{array}$ & $\begin{array}{l}\text { Kontrollgruppe } \\
\text { Therapiegruppen }\end{array}$ & $\begin{array}{l}8 \\
8\end{array}$ & $\begin{array}{r}94,75 \\
108,14\end{array}$ & $\begin{array}{r}9,28 \\
11,62\end{array}$ & ,028 \\
\hline
\end{tabular}

$\mathrm{N}=$ Anzahl der Probanden; $\mathrm{MW}=$ Mittelwert; $\mathrm{SD}=$ Standardabweichung ; $\mathrm{msec}=$ Millisekunden; $\mathrm{sec}=\mathrm{Sekunden}$ 
Der Leistungsvergleich zwischen den PD-Patienten und den gesunden Probanden der Therapiebedingung in der 2. neuropsychologischen Untersuchung ergab keine signifikanten Unterschiede hinsichtlich der basalen Aufmerksamkeit „Alertness ohne Warnton“, der Steigerungsfähigkeit der Aufmerksamkeit „Alertness mit Warnton“, der kognitiven Schnelligkeit (TMT-A) oder der Konzentrationsleistungen, gemessen mit dem Subtest „Konzentration“ aus der WMS-R; (Tab. 11).

Tab.11 Mittelwerte der Aufmerksamkeitsleistungen der PD-Patienten und gesunden VPN in Therapiebedingung in der 2. neuropsychologischen Untersuchung, gemessen mit TAP-Alertness, TMT-A und WMS-R-Subtest „Aufmerksamkeit und Konzentration“

\begin{tabular}{|c|c|c|c|c|c|}
\hline $\begin{array}{l}\text { 2.neuropsychologische } \\
\text { Untersuchung } \\
\text { Testverfahren }\end{array}$ & $\begin{array}{c}\text { VPN } \\
\text { Therapiebedingung }\end{array}$ & $\mathrm{N}$ & MW & SD & $\begin{array}{l}\text { Signifikanz } \\
\text { 2-seitig }\end{array}$ \\
\hline $\begin{array}{l}\text { Alertness } \\
\text { ohne Warnton (TAP) }\end{array}$ & $\begin{array}{l}\text { Gesunde VPN } \\
\text { PD-Patienten }\end{array}$ & 8 & $\begin{array}{l}248,71 \mathrm{msec} \\
269,22 \mathrm{msec}\end{array}$ & $\begin{array}{l}22,35 \mathrm{msec} \\
57,11 \mathrm{msec}\end{array}$ & 346, \\
\hline $\begin{array}{l}\text { Alertness } \\
\text { mit Warnton (TAP) }\end{array}$ & $\begin{array}{l}\text { Gesunde VPN } \\
\text { PD-Patienten }\end{array}$ & $\begin{array}{l}8 \\
8\end{array}$ & $\begin{array}{l}232,00 \mathrm{msec} \\
249,55 \mathrm{msec}\end{array}$ & $\begin{array}{l}26,88 \mathrm{msec} \\
51,59 \mathrm{msec}\end{array}$ & 396, \\
\hline $\begin{array}{l}\text { Trail-Making-Test-A } \\
\text { (TMT-A) }\end{array}$ & $\begin{array}{l}\text { Gesunde VPN } \\
\text { PD-Patienten }\end{array}$ & $\begin{array}{l}8 \\
8\end{array}$ & $\begin{array}{l}36,42 \mathrm{sec} \\
56,00 \mathrm{sec}\end{array}$ & $\begin{array}{r}8,07 \mathrm{sec} \\
30,65 \mathrm{sec}\end{array}$ & 098, \\
\hline $\begin{array}{l}\text { Aufmerksamkeit, } \\
\text { Konzentration (WMS-R) }\end{array}$ & $\begin{array}{l}\text { Gesunde VPN } \\
\text { PD-Patienten }\end{array}$ & $\begin{array}{l}8 \\
8\end{array}$ & $\begin{array}{l}108,14 \\
105,55\end{array}$ & $\begin{array}{l}11,62 \\
20,54\end{array}$ & ,771 \\
\hline
\end{tabular}

$\mathrm{N}=$ Anzahl der Probanden; $\mathrm{MW}=$ Mittelwert; $\mathrm{SD}=$ Standardabweichung; $\mathrm{msec}=$ Millisekunden; $\mathrm{sec}=$ Sekunden

Im Vergleich zu den gesunden Probanden erzielten die PD-Patienten. der Kontrollbedingung in der 2. neuropsychologischen Untersuchung signifikant lansamere Reaktionszeiten in der basalen Aufmerksamkeitsfähigkeit „Alertness ohne Warnton“, wobei die PD-Patienten hier einen Mittelwert (MW) von 296,62msec, SD=31,89msec und die gesunden VPN der Kontrollgruppe einen $\mathrm{MW}=252,00 \mathrm{msec}, \mathrm{SD}=34,92 \mathrm{msec}$; $\mathrm{p}$.05 erreichten; (Abb. 3, Tab. 12). 


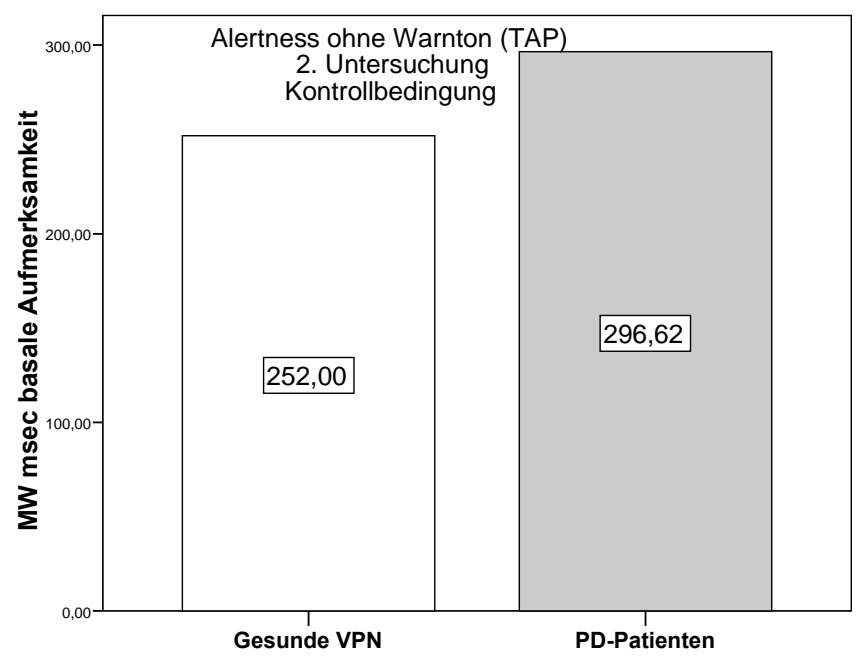

Abb.3 Basale Aufmerksamkeitsleistungen der gesunden VPN und PD-Patienten der Kontrollbedingung in der 2. Untersuchung $(p=0,018)$

Demgegenüber konnten in der Steigerungsfähigkeit der Aufmerksamkeit, als auch in der kognitiven Schnelligkeit sowie der Konzentrationsfähigkeit keine weiteren signifikanten Unterschiede zwischen den gesunden Probanden und den PD-Patienten der Kontrollgruppe festgestellt werden; (Tab. 12).

Tab.12 Mittelwerte der Aufmerksamkeitsleistungen der PD-Patienten und gesunden VPN der Kontrollbedingung in der 2. neuropsychologischen Untersuchung, gemessen mit TAP-Alertness, TMT-A und Subtest WMS-R „Aufmerksamkeit und Konzentration“

\begin{tabular}{|c|c|c|c|c|c|}
\hline $\begin{array}{l}\text { 2.neuropsychologische } \\
\text { Untersuchung } \\
\text { Testverfahren }\end{array}$ & $\begin{array}{c}\text { VPN } \\
\text { Kontrollgruppe }\end{array}$ & $\mathrm{N}$ & MW & SD & $\begin{array}{l}\text { Signifikanz } \\
\text { 2-seitig }\end{array}$ \\
\hline \multirow{2}{*}{$\begin{array}{l}\text { Alertness } \\
\text { ohne Warnton (TAP) }\end{array}$} & Gesunde VPn & 8 & $252,00 \mathrm{msec}$ & $34,92 \mathrm{msec}$ & \multirow{2}{*}{,018 } \\
\hline & PD-Patienten & 8 & $296,62 \mathrm{msec}$ & $31,89 \mathrm{msec}$ & \\
\hline \multirow{2}{*}{$\begin{array}{l}\text { Alertness } \\
\text { mit Warnton (TAP) }\end{array}$} & Gesunde VPn & 8 & $234,50 \mathrm{msec}$ & $38,39 \mathrm{msec}$ & \multirow{2}{*}{,415 } \\
\hline & PD-Patienten & 8 & $249,50 \mathrm{msec}$ & $32,78 \mathrm{msec}$ & \\
\hline \multirow{2}{*}{$\begin{array}{l}\text { Trail-Making-Test-A } \\
\text { (TMT-A) }\end{array}$} & Gesunde VPn & 8 & $43,62 \mathrm{sec}$ & $13,19 \mathrm{sec}$ & \multirow{2}{*}{,279 } \\
\hline & PD-Patienten & 8 & $53,25 \mathrm{sec}$ & $20,08 \mathrm{sec}$ & \\
\hline \multirow{2}{*}{$\begin{array}{l}\text { Aufmerksamkeit, } \\
\text { Konzentration (WMS-R) }\end{array}$} & Gesunde VPn & 8 & 94,75 & 9,28 & \multirow{2}{*}{,381 } \\
\hline & PD-Patienten & 8 & 89,62 & 13,07 & \\
\hline
\end{tabular}

$\mathrm{N}=$ =Anzahl der Probanden; MW=Mittelwert; $\mathrm{SD}=$ Standardabweichung; $\mathrm{msec}=$ Millisekunden; $\mathrm{sec}=\mathrm{Sekunden}$ 


\subsubsection{Vergleich der Aufmerksamkeits- und Konzentrationsleistungen in der 1. und 2. Untersuchung}

Der T-Test für gepaarte Stichproben ergab ferner, dass diejenigen PD-Patienten, die an der Therapie teilnahmen, in der zweiten neuropsychologischen Untersuchung im Vergleich zur ersten Untersuchung eine signifikante Verbesserung im Bereich der basalen Aufmerksamkeitsleistung (TAP „Alertness ohne Warnton) (MW vorher $=318,33 \mathrm{msec} ; \mathrm{SD}=52,35 \mathrm{msec} M W$ nachher $=269,22 \mathrm{msec}, \mathrm{SD}=57,11 \mathrm{msec} ; \mathrm{p}<.05)$ aufwiesen. Die Steigerungsfähigkeit der Aufmerksamkeit (TAP „Alertness mit Warnton“) konnte in dieser Gruppe ebenfalls signifikant verbessert werden (MW vorher $=287,11 \mathrm{msec}$, $\mathrm{SD}=54,66 \mathrm{msec}$; $M W$ nachher $=249,50 \mathrm{msec}, \mathrm{SD}=51,59 \mathrm{msec}$; $\mathrm{p}<0.05$ ); (Abb. $4 \mathrm{u}$. 5; Tab. 13).

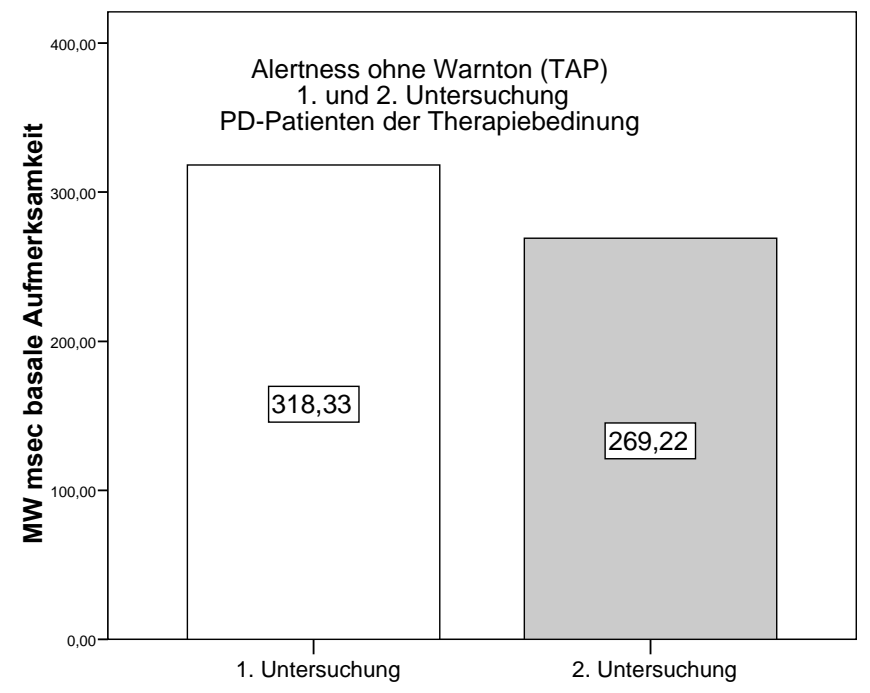

Abb. 4 Basale Aufmerksamkeitsleistung der PD-Patienten in Therapiebedingung in der 1 . und 2 . Untersuchung $(p=0,021)$ 


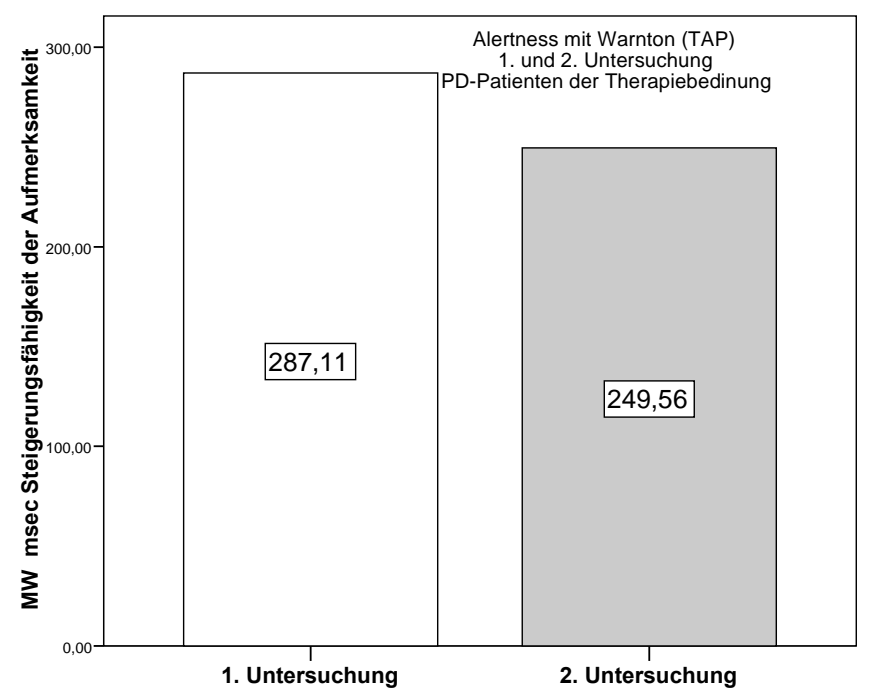

Abb. 5 Steigerungsfähigkeit der Aufmerksamkeit der PD-Patienten in Therapiebedingung in der 1. und 2. Untersuchung $(p=0,048)$

Die kognitive Schnelligkeit (TMT-A) verbesserte sich in der Gruppe der PDPatienten in Therapiebedingung nur tendenziell. Auch die Konzentrationsleistung (WMS-R-Subtest) verbesserte sich, was jedoch nicht als signifikant einzustufen war; (Tab. 13).

Tab.13 Mittelwerte der Aufmerksamkeitsleistungen der PD-Patienten in Therapiebedingung in der 1. und 2. neuro psychologischen Untersuchung, gemessen mit TAP-Alertness, TMT-A und WMS- $R$ Subtest „Aufmerksamkeit und Konzentration“ aus WMS-R

\begin{tabular}{|l|r|r|r|r|}
\hline \multicolumn{1}{|c}{$\begin{array}{c}\text { 1. und 2. } \\
\text { neuropsychologische Untersuchung } \\
\text { Testverfahren }\end{array}$} & $\begin{array}{r}\text { PD-Patienten } \\
\text { Therapiegruppen } \\
\text { N }\end{array}$ & MW & SD & $\begin{array}{c}\text { Signifikanz } \\
\text { 2-seitig }\end{array}$ \\
\hline Alertness ohne Warnton (TAP) vorher & 8 & $318,33 \mathrm{msec}$ & $52,35 \mathrm{msec}$ &, 021 \\
Alertness ohne Warnton (TAP) nachher & 8 & $269,22 \mathrm{msec}$ & $57,11 \mathrm{msec}$ &, 048 \\
\hline Alertness mit Warnton (TAP) vorher & 8 & $287,11 \mathrm{msec}$ & $54,66 \mathrm{msec}$ &, 144 \\
Alertness mit Warnton (TAP) nachher & 8 & $249,55 \mathrm{msec}$ & $51,59 \mathrm{msec}$ & \\
\hline Trail Making Test A (TMT-A) vorher & 8 & $64,66 \mathrm{sec}$ & $23,62 \mathrm{sec}$ &, 119 \\
Trail Making Test A (TMT-A) nachher & 8 & $56,00 \mathrm{sec}$ & $30,65 \mathrm{sec}$ & \\
\hline Konzentration (WMS-R) vorher & 8 & 95,44 & 11,71 & \\
Konzentration (WMS-R) nachher & 8 & 105,55 & 20,54 & \\
\hline
\end{tabular}

$\mathrm{N}=$ Anzahl der Probanden; $\mathrm{MW}=$ Mittelwert; $\mathrm{SD}=$ Standardabweichung; $\mathrm{msec}=$ Millisekunden; $\mathrm{sec}=$ Sekunden 
Die PD-Patienten der Kontrollgruppe verschlechterten sich nach 4 Monaten in der basalen Aufmerksamkeit (TAP „Alertness ohne Warton“) signifikant (MW vorher = 269,37msec, SD=22,48msec; MW nachher = 296,62msec, SD=31,02msec; p<.05); (Abb. 6, Tab. 14).

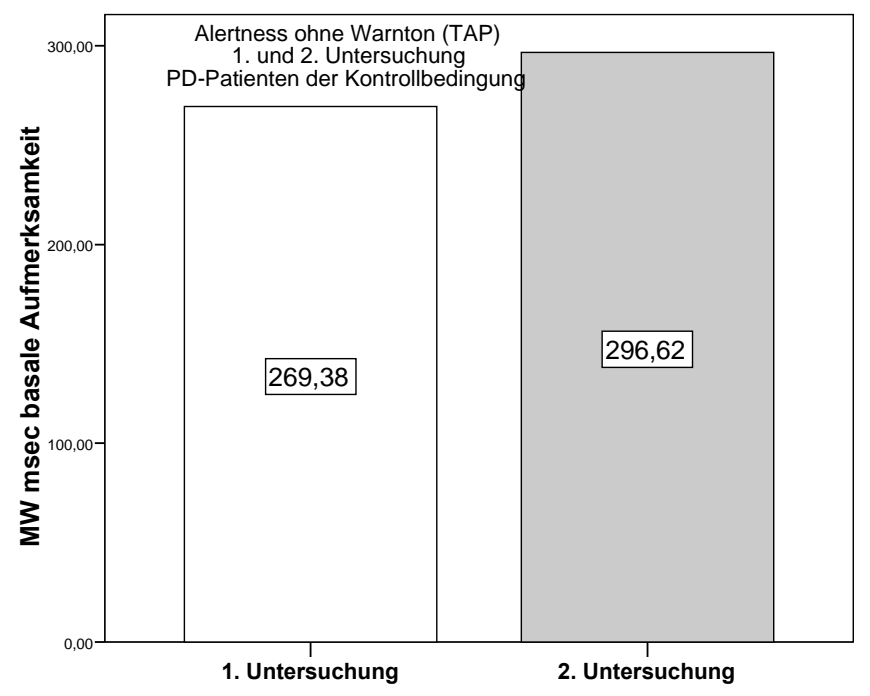

Abb.6 Basale Aufmerksamkeit der PD-Patienten in Kotrollbedingung in der 1. und 2. Untersuchung $(p=0,45)$

Die Konzentrationsfähigkeit (WMS-R-Subtest) der PD-Patienten dieser Gruppe (MW vorher =101,12 Punkte; SD=15,16 Punkte; MW nachher = 89,62 Punkte, $S D=13,07$ Punkte; $p<0,02$ ) hat sich ebenfalls signifikant verschlechtert; (Abb. 7, Tab. 14). 


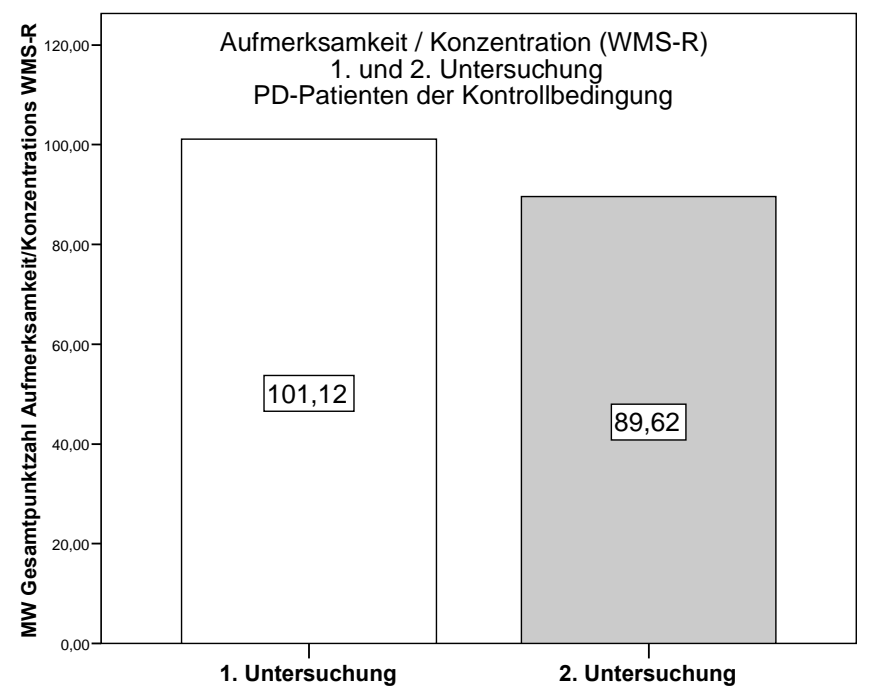

Abb.7 Aufmerksamkeit und Konzentrationsleistungen der PD-Patienten in Kontrollbedingung in der 1. und 2. Untersuchung $(p=0,02)$

Die Überprüfung der Steigerungsfähigkeit der Aufmerksamkeit und der kognitiven Schnelligkeit ergab nach 4 Monaten keine signifikante Veränderung der Werte (Tab. 14).

Tab.14 Mittelwerte der Aufmerksamkeitsleistungen der PD-Patienten in Kontrollbedingung in der 1.und 2.neuropsychologischen Untersuchung, gemessen mit TAP-Alertness, TMTA und Subtest WMS-R „Aufmerksamkeit und Konzentration“

\begin{tabular}{|l|r|c|c|c|}
\hline \multicolumn{1}{|c|}{$\begin{array}{c}\text { 1.und 2. } \\
\text { neuropsychologische Untersuchung } \\
\text { Testverfahren }\end{array}$} & $\begin{array}{c}\text { PD-Patienten } \\
\text { Kontrollgruppe } \\
\text { N }\end{array}$ & MW & SD & $\begin{array}{c}\text { Signifikanz } \\
\text { 2-seitig }\end{array}$ \\
\hline Alertness ohne Warnton (TAP) vorher & 8 & $269,37 \mathrm{msec}$ & $22,48 \mathrm{msec}$ &, 045 \\
Alertness ohne Warnton (TAP) nachher & 8 & $296,62 \mathrm{msec}$ & $31,02 \mathrm{msec}$ &, 987 \\
\hline Alertness mit Warnton (TAP) vorher & 8 & $249,25 \mathrm{msec}$ & $26,87 \mathrm{msec}$ &, 092 \\
Alertness mit Warnton (TAP) nachher & 8 & $249,50 \mathrm{msec}$ & $32,78 \mathrm{msec}$ & \\
\hline Trail Making Test A (TMT-A) vorher & 8 & $65,12 \mathrm{sec}$ & $21,35 \mathrm{sec}$ & 0,02 \\
Trail Making Test A (TMT-A) nachher & 8 & $53,25 \mathrm{sec}$ & $20,08 \mathrm{sec}$ & \\
\hline Konzentration ((WMS-R) vorher & 8 & 101,12 & 15,16 & \\
Konzentration ( WMS-R) nachher & 8 & 89,62 & 13,07 & \\
\hline
\end{tabular}

$\mathrm{N}=$ Anzahl der Probanden; $\mathrm{MW}=$ Mittelwert; $\mathrm{SD}=$ Standardabweichung; $\mathrm{msec}=$ Millisekunden; $\mathrm{sec}=$ Sekunden

Der T-Test für gepaarte Stichproben ergab in der zweiten neuropsychologischen Untersuchung im Vergleich zur ersten Untersuchung für die gesunden VPN, die 
an der Therapie teilnahmen eine signifikante Verbesserung der kognitiven Schnelligkeit $(M W$ vorher $=47,00 \mathrm{sec}, \mathrm{SD}=16,32$; MW nachher $=36,42 \mathrm{sec}$, $\mathrm{SD}=8,07 \mathrm{sec} ; \mathrm{p}<0.05$ ); und der Konzentration (MW vorher = 85,14 Punkte, $\mathrm{SD}=$ 11,83 Punkte; MW nachher = 108,14 Punkte, $S D=11,62$ Punkte; $p<, 01$ ); (Abb. 8, 9; Tab. 15).

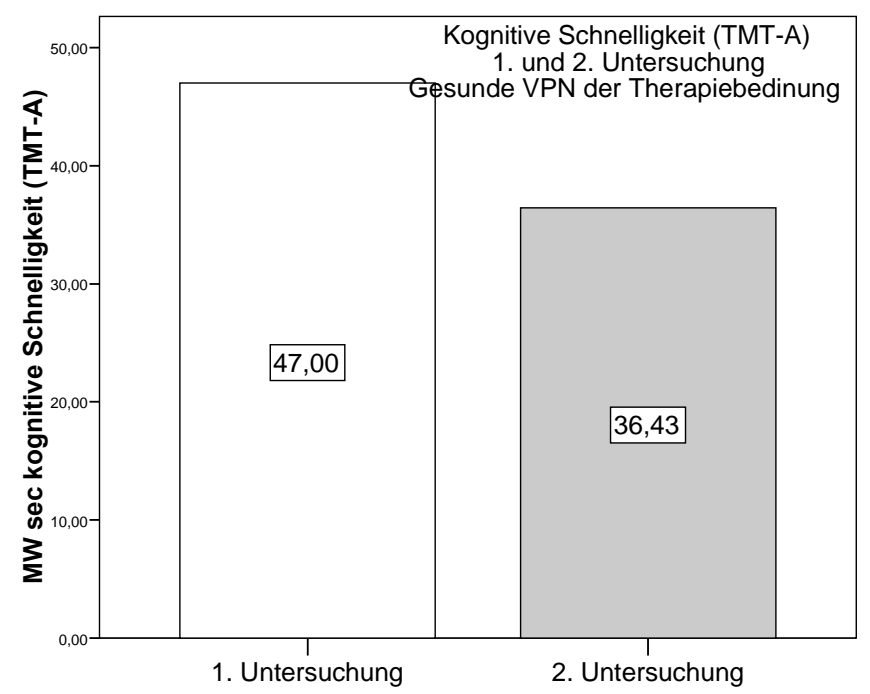

Abb.8 Kognitive Schnelligkeit der gesunden VPN in Therapiebedingung in der 1 . und 2 . Untersuchung $(p=0,043)$

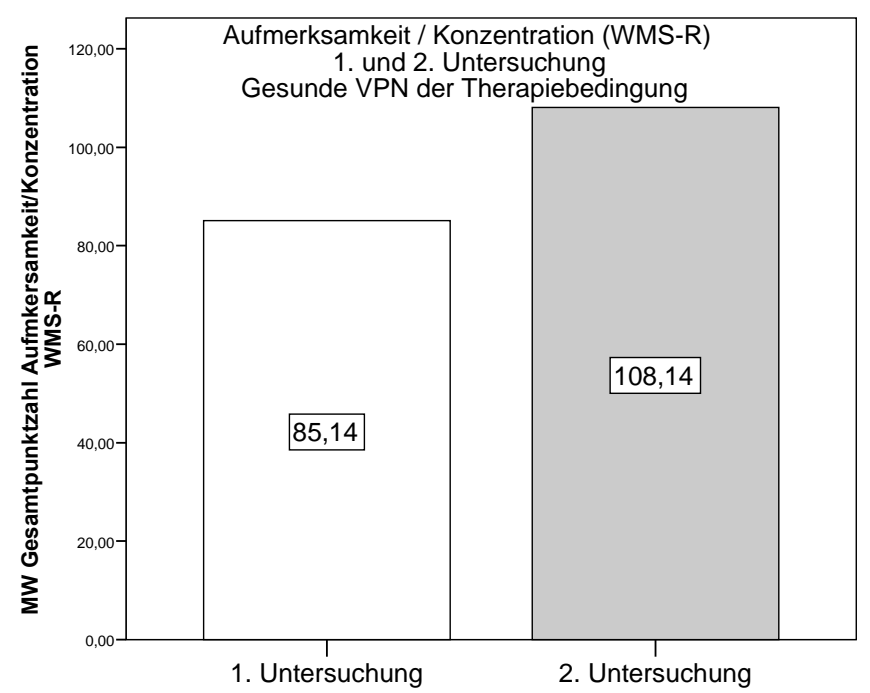

Abb. 9 Aufmerksamkeits- und Konzentrationsfähigkeit der gesunden VPN in Therapiebedingung in der 1. und 2. Untersuchung $(p=0,009)$ 
Keine Leistungsverbesserungen zeigten sich im Bereich der basalen Aufmerksamkeitsleistung und der Steigerungsfähigkeit der Aufmerksamkeit; (Tab. 15).

Tab.15 Mittelwerte der Aufmerksamkeitsleistungen der gesunden VPN der Therapiebedingung in der 1.und 2. neuropsychologischen Untersuchung, gemessen mit TAP-Alertness, TMT-A und Subtest WMS-R „Konzentration"

\begin{tabular}{|c|c|c|c|c|}
\hline $\begin{array}{c}\text { 1.und } 2 . \\
\text { neuropsychologische Untersuchung } \\
\text { Testverfahren }\end{array}$ & $\begin{array}{l}\text { Gesunde VPN } \\
\text { Therapiegruppen } \\
\mathrm{N}\end{array}$ & MW & SD & $\begin{array}{c}\text { Signifikanz } \\
\text { 2-seitig }\end{array}$ \\
\hline $\begin{array}{l}\text { Alertness ohne Warnton (TAP) vorher } \\
\text { Alertness ohne Warnton (TAP) nachher }\end{array}$ & 8 & $\begin{array}{l}259,57 \mathrm{msec} \\
248,71 \mathrm{msec}\end{array}$ & $\begin{array}{l}21,77 \mathrm{msec} \\
22,35 \mathrm{msec}\end{array}$ & 301 \\
\hline $\begin{array}{l}\text { Alertness mit Warnton (TAP) vorher } \\
\text { Alertness mit Warnton (TAP) nachher }\end{array}$ & 8 & $\begin{array}{l}246,57 \mathrm{msec} \\
232,00 \mathrm{msec}\end{array}$ & $\begin{array}{l}35,20 \mathrm{msec} \\
26,88 \mathrm{msec}\end{array}$ & 159, \\
\hline $\begin{array}{l}\text { Trail-Making-Test-A (TMT-A) vorher } \\
\text { Trail-Making-Test-A (TMT-A) nachher }\end{array}$ & $\begin{array}{l}8 \\
8\end{array}$ & $\begin{array}{l}47,00 \mathrm{sec} \\
36,42 \mathrm{sec}\end{array}$ & $\begin{array}{r}16,23 \mathrm{sec} \\
8,07 \mathrm{sec}\end{array}$ & ,043 \\
\hline $\begin{array}{l}\text { Konzentration (WMS-R) vorher } \\
\text { Konzentration (WMS-R) nachher }\end{array}$ & 8 & $\begin{array}{r}85,14 \\
108,14\end{array}$ & $\begin{array}{r}11,83 \\
11,62\end{array}$ & ,009 \\
\hline
\end{tabular}

$\mathrm{N}=$ Anzahl der Probanden; MW=Mittelwert; $\mathrm{SD}=$ Standardabweichung; $\mathrm{msec}=$ Millisekunden; $\mathrm{sec}=$ Sekunden

Die nachfolgende Tabelle zeigt, dass die gesunden VPN der Kontrollgruppe in der zweiten neuropsychologischen Untersuchung insgesamt keine Veränderungen hinsichtlich der Konzentrations- und Aufmerksamkeitsleistungen erzielen konnten; (Tab. 16).

Tab.16 Mittelwerte der Aufmerksamkeitsleistungen der gesunden VPN in Kontrollbedingung in der 1. und 2. neuropsychologischen Untersuchung, gemessen mit TAP-Alertness, TMT-A und Subtest „Konzentration“ WMS-R

\begin{tabular}{|c|c|c|c|c|}
\hline $\begin{array}{l}\text { 1.und } 2 . \\
\text { neuropsychologische Untersuchung } \\
\text { Testverfahren }\end{array}$ & $\begin{array}{l}\text { Gesunde VPN } \\
\text { Kontrollgruppe } \\
\text { N }\end{array}$ & MW & SD & $\begin{array}{c}\text { Signifikanz } \\
\text { 2-seitig }\end{array}$ \\
\hline $\begin{array}{l}\text { Alertness ohne Warnton (TAP) vorher } \\
\text { Alertness ohne Warnton (TAP) nachher }\end{array}$ & $\begin{array}{l}8 \\
8\end{array}$ & $\begin{array}{l}245,50 \mathrm{msec} \\
252,00 \mathrm{msec}\end{array}$ & $\begin{array}{l}33,42 \mathrm{msec} \\
34,92 \mathrm{msec}\end{array}$ & 455, \\
\hline $\begin{array}{l}\text { Alertness mit Warnton (TAP) vorher } \\
\text { Alertness mit Warnton (TAP) nachher }\end{array}$ & 8 & $\begin{array}{l}231,87 \mathrm{msec} \\
234,50 \mathrm{msec}\end{array}$ & $\begin{array}{l}36,11 \mathrm{msec} \\
38,39 \mathrm{msec}\end{array}$ & ,521 \\
\hline $\begin{array}{l}\text { Trail-Making-Test-A (TMT-A) vorher } \\
\text { Trail-Making-Test-A (TMT-A) nachher }\end{array}$ & $\begin{array}{l}8 \\
8\end{array}$ & $\begin{array}{l}49,37 \mathrm{sec} \\
43,62 \mathrm{sec}\end{array}$ & $\begin{array}{l}15,72 \mathrm{sec} \\
13,19 \mathrm{sec}\end{array}$ & 178, \\
\hline $\begin{array}{l}\text { Konzentration (WMS-R) vorher } \\
\text { Konzentration (WMS-R) nachher }\end{array}$ & $\begin{array}{l}8 \\
8\end{array}$ & 98,25 & 13,34 & 471 \\
\hline
\end{tabular}

$\mathrm{N}=$ Anzahl der Probanden; MW=Mittelwert; $\mathrm{SD}=$ Standardabweichung; $\mathrm{msec}=$ Millisekunden; $\mathrm{sec}=$ Sekunden 


\subsection{Gedächtnisleistungen}

\subsubsection{Gedächtnisleistungen in der 1. Untersuchung}

In der 1. neuropsychologischen Untersuchung unterschieden sich die PDPatienten der Therapie- bzw. Kontrollbedingung in allen untersuchten Gedächtnisbereichen nicht signifikant voneinander. Die Leistungen in der verbalen Merkspanne, gemessen mit der Wechsler-Memory-Scale-R (WMS-R Subtest „Zahlennachsprechen vorwärts") und in der non-verbalen Merkspanne, gemessen mit der Wechsler-Memory-Scale-R (WMS-R Subtest „Corsiblock vorwärts") waren ähnlich ausgeprägt. Auch im Bereich des verbalen und des non-verbalen Kurzzeitgedächtnisses unterschieden sich die PD-Patienten der Therapie- bzw. Kontrollbedingung, gemessen mit der Wechsler-Memory-Scale-R (WMS-R „Verbales Gedächtnis“ und „Visuelles Gedächtnis) nicht signifikant voneinander. Die allgemeine Leistungsfähigkeit des Kurzzeitgedächtnisses und des Langzeitgedächtnisses, gemessen mit der Wechsler-Memory-Scale-R (WMS-R "Allgemeines Kurzzeitgedächtnis“ und „Verzögerte Wiedergabe“) waren gleich ausgeprägt; (Tab. 17).

Tab.17 Mittelwerte der Gedächtnisleistungen PD-Patienten in Kontroll- und Therapiebedingung in der 1. neuropsychologischen Untersuchung, gemessen mit WMS-R-Subtests

\begin{tabular}{|c|c|c|c|c|c|}
\hline $\begin{array}{c}\text { 1. neuropsychologische } \\
\text { Untersuchung }\end{array}$ & PD-Patienten & $\mathrm{N}$ & MW & SD & $\begin{array}{l}\text { Signifikanz } \\
\text { 2-seitig }\end{array}$ \\
\hline Verbale Merkspanne WMS-R & $\begin{array}{l}\text { Kontrollgruppe } \\
\text { Therapiegruppen }\end{array}$ & $\begin{array}{l}8 \\
8\end{array}$ & $\begin{array}{l}7,62 \\
7,88\end{array}$ & $\begin{array}{l}1,76 \\
1,76\end{array}$ & ,763 \\
\hline $\begin{array}{l}\text { Non-verbale } \\
\text { Merkspanne WMS-R }\end{array}$ & $\begin{array}{l}\text { Kontrollgruppe } \\
\text { Therapiegruppen }\end{array}$ & $\begin{array}{l}8 \\
8\end{array}$ & $\begin{array}{l}7,37 \\
6,88\end{array}$ & $\begin{array}{r}, 91 \\
1,05\end{array}$ & ,329 \\
\hline $\begin{array}{l}\text { Verbales Kurzzeitgedächtnis } \\
\text { WMS-R }\end{array}$ & $\begin{array}{l}\text { Kontrollgruppe } \\
\text { Therapiegruppen }\end{array}$ & $\begin{array}{l}8 \\
8\end{array}$ & $\begin{array}{l}96,75 \\
94,66\end{array}$ & $\begin{array}{l}17,87 \\
15,19\end{array}$ & ,799 \\
\hline $\begin{array}{l}\text { Visuelles Kurzzeitgedächtnis } \\
\text { WMS-R }\end{array}$ & $\begin{array}{l}\text { Kontrollgruppe } \\
\text { Therapiegruppen }\end{array}$ & $\begin{array}{l}8 \\
8\end{array}$ & $\begin{array}{r}102,00 \\
95,11\end{array}$ & $\begin{array}{r}19,75 \\
9,87\end{array}$ & ,369 \\
\hline $\begin{array}{l}\text { Allgemeines Kurzzeitgedächtnis } \\
\text { WMS-R }\end{array}$ & $\begin{array}{l}\text { Kontrollgruppe } \\
\text { Therapiegruppen }\end{array}$ & $\begin{array}{l}8 \\
8\end{array}$ & $\begin{array}{l}98,75 \\
94,66\end{array}$ & $\begin{array}{l}17,05 \\
13,46\end{array}$ &, 590 \\
\hline Verzögerte Wiedergabe WMS-R & $\begin{array}{l}\text { Kontrollgruppe } \\
\text { Therapiegruppen }\end{array}$ & $\begin{array}{l}8 \\
8\end{array}$ & $\begin{array}{l}106,12 \\
101,55\end{array}$ & $\begin{array}{l}15,40 \\
10,84\end{array}$ & ,486 \\
\hline
\end{tabular}

$\mathrm{N}=$ Anzahl der Probanden; MW=Mittelwert; SD=Standardabweichung 
Auch die gesunden VPN der Therapie- bzw. Kontrollbedingung unterschieden sich in ihren Gedächtnisleistungen in der 1. neuropsychologischen Untersuchung nicht voneinander. Im Hinblick auf die verbale und non-verbale Merkspanne, dem verbalen und non-verbalen Kurzzeitgedächtnis sowie dem Langzeitdedächtnis zeigten sich keine signifikanten Unterschiede zwischen den Gruppen; (Tab. 18).

Tab.18 Mittelwerte der Gedächtnisleistungen der gesunden VPN in Kontroll- und Therapiebedingung in der 1. neuropsychologischen Untersuchung, gemessen mit WMS-R-Subtests

\begin{tabular}{|c|c|c|c|c|c|}
\hline $\begin{array}{l}\text { 1. neuropsychologische } \\
\text { Untersuchung } \\
\text { Testverfahren }\end{array}$ & Gesunde VPN & $\mathrm{N}$ & MW & SD & $\begin{array}{c}\text { Signifikanz } \\
\text { 2-seitig }\end{array}$ \\
\hline \multirow{2}{*}{$\begin{array}{l}\text { Verbale Merkspanne } \\
\text { WMS-R }\end{array}$} & Kontrollgruppe & 8 & 7,87 & 1,88 & \multirow{2}{*}{,225 } \\
\hline & Therapiegruppen & 8 & 6,71 & 1,60 & \\
\hline \multirow{2}{*}{$\begin{array}{l}\text { Visuelle Merkspanne } \\
\text { WMS-R }\end{array}$} & Kontrollgruppe & 8 & 6,87 & 1,88 & \multirow{2}{*}{,411 } \\
\hline & Therapiegruppen & 8 & 7,57 & 1,13 & \\
\hline \multirow{2}{*}{$\begin{array}{l}\text { Verbales Gedächtnis } \\
\text { WMS-R }\end{array}$} & Kontrollgruppe & 8 & 94,37 & 13,79 & \multirow{2}{*}{,267 } \\
\hline & Therapiegruppen & 8 & 103,71 & 17,38 & \\
\hline \multirow{2}{*}{$\begin{array}{l}\text { Visuelles Gedächtnis } \\
\text { WMS-R }\end{array}$} & Kontrollgruppe & 8 & 99,25 & 16,73 & \multirow{2}{*}{,718 } \\
\hline & Therapiegruppen & 8 & 96,42 & 12,03 & \\
\hline \multirow{2}{*}{$\begin{array}{l}\text { Allgem. Gedächtnis } \\
\text { WMS-R }\end{array}$} & Kontrollgruppe & 8 & 96,25 & 15,83 & \multirow{2}{*}{, 569} \\
\hline & Therapiegruppen & 8 & 100,57 & 12,24 & \\
\hline \multirow{2}{*}{$\begin{array}{l}\text { Verzögerte Wiedergabe } \\
\text { WMS-R }\end{array}$} & Kontrollgruppe & 8 & 102,25 & 14,41 & \multirow{2}{*}{, 592} \\
\hline & Therapiegruppen & 8 & 106,00 & 11,59 & \\
\hline
\end{tabular}

$\mathrm{N}=$ Anzahl der Probanden; $\mathrm{MW}=$ Mittelwert; $\mathrm{SD}=$ Standardabweichung

Zwischen den PD-Patienten und der gesunden VPN der Therapiebedingung fand sich im Hinblick auf die verbale und non-verbale Merkspanne, dem verbalen und non-verbalen Kurzzeitgedächtnis sowie dem Langzeitgedächtnis zu Beginn der Therapie kein signifikanter Leistungsunterschied; (Tab. 19). 
Tab.19 Mittelwerte der Gedächtnisleistungen der PD-Patienten und der gesunden VPN in Therapiebedingung in der 1. neuropsychologischen Untersuchung, gemessen mit WMS-R-Subtests

\begin{tabular}{|c|c|c|c|c|c|}
\hline $\begin{array}{l}\text { 1. neuropsychologische } \\
\text { Untersuchung } \\
\text { Testverfahren }\end{array}$ & $\begin{array}{c}\text { VPN } \\
\text { Therapiegruppen }\end{array}$ & $\mathrm{N}$ & MW & SD & $\begin{array}{l}\text { Signifikanz } \\
\text { 2-seitig }\end{array}$ \\
\hline \multirow{2}{*}{$\begin{array}{l}\text { Verbale Merkspanne } \\
\text { WMS-R }\end{array}$} & Gesunde VPN & 8 & 6,71 & 1,60 & \multirow{2}{*}{,191 } \\
\hline & PD-Patienten & 8 & 7,88 & 1,76 & \\
\hline \multirow{2}{*}{$\begin{array}{l}\text { Visuelle Merkspanne } \\
\text { WMS-R }\end{array}$} & Gesunde VPN & 8 & 7,57 & 1,13 & \multirow[b]{2}{*}{,234 } \\
\hline & PD-Patienten & 8 & 6,88 & 1,05 & \\
\hline \multirow{2}{*}{$\begin{array}{l}\text { Verbales Gedächtnis } \\
\text { WMS-R }\end{array}$} & Gesunde VPN & 8 & 103,71 & 17,38 & \multirow{2}{*}{,286 } \\
\hline & PD-Patienten & 8 & 94,66 & 15,19 & \\
\hline \multirow{2}{*}{$\begin{array}{l}\text { Visuelles Gedächtnis } \\
\text { WMS-R }\end{array}$} & Gesunde VPN & 8 & 96,42 & 12,03 & \multirow{2}{*}{,813 } \\
\hline & PD-Patienten & 8 & 95,11 & 9,87 & \\
\hline \multirow{2}{*}{$\begin{array}{l}\text { Allgem. Gedächtnis } \\
\text { WMS-R }\end{array}$} & Gesunde VPN & 8 & 100,57 & 12,24 & \multirow{2}{*}{,381 } \\
\hline & PD-Patienten & 8 & 94,66 & 13,46 & \\
\hline \multirow{2}{*}{$\begin{array}{l}\text { Verzögerte Wiedergabe } \\
\text { WMS-R }\end{array}$} & Gesunde VPN & 8 & 106,00 & 11,59 & \multirow{2}{*}{,443 } \\
\hline & PD-Patienten & 8 & 101,55 & 10,84 & \\
\hline
\end{tabular}

$\mathrm{N}=$ Anzahl der Probanden; MW=Mittelwert; SD=Standardabweichung

Die Ergebnisse der 1. neuropsychologischen Untersuchung der PD-Patienten und der gesunden VPN der Kontrollbedingung hinsichtlich ihrer Gedächtnisleistungen waren als nicht signifikant unterschiedlich zu bewerten, wobei zu beachten ist, dass die PD-Patienten, abgesehen von der verbalen Merkspanne, tendenziell bessere Leistungen aufwiesen; (Tab.20).

Tab.20 Mittelwerte der Gedächtnisleistungen der PD-Patienten und der gesunden VPN in Kontrollbedingung in der 1. neuropsychologischen Untersuchung, gemessen mit WMS-R-Subtests

\begin{tabular}{|c|c|c|c|c|c|}
\hline $\begin{array}{l}\text { 1. neuropsychologische } \\
\text { Untersuchung } \\
\text { Testverfahren }\end{array}$ & $\begin{array}{c}\text { VPN } \\
\text { Kontrollgruppe }\end{array}$ & $\mathrm{N}$ & MW & SD & $\begin{array}{l}\text { Signifikanz } \\
\text { 2-seitig }\end{array}$ \\
\hline \multirow{2}{*}{$\begin{array}{l}\text { Verbale Merkspanne } \\
\text { WMS-R }\end{array}$} & Gesunde VPn & 8 & 7,87 & 1,88 & \multirow{2}{*}{,788 } \\
\hline & PD-Patienten & 8 & 7,62 & 1,76 & \\
\hline \multirow{2}{*}{$\begin{array}{l}\text { Visuelle Merkspanne } \\
\text { WMS-R }\end{array}$} & Gesunde VPn & 8 & 6,87 & 1,88 & \multirow{2}{*}{,511 } \\
\hline & PD-Patienten & 8 & 7,37 & ,91 & \\
\hline \multirow{2}{*}{$\begin{array}{l}\text { Verbales Gedächtnis } \\
\text { WMS-R }\end{array}$} & Gesunde VPn & 8 & 94,37 & 13,79 & \multirow{2}{*}{,770 } \\
\hline & PD-Patienten & 8 & 96,75 & 17,87 & \\
\hline \multirow{2}{*}{$\begin{array}{l}\text { Visuelles Gedächtnis } \\
\text { WMS-R }\end{array}$} & Gesunde VPn & 8 & 99,25 & 16,73 & \multirow{2}{*}{,768 } \\
\hline & PD-Patienten & 8 & 102,00 & 19,75 & \\
\hline \multirow{2}{*}{$\begin{array}{l}\text { Allgem. Gedächtnis } \\
\text { WMS-R }\end{array}$} & Gesunde VPn & 8 & 96,25 & 15,83 & \multirow{2}{*}{,766 } \\
\hline & PD-Patienten & 8 & 98,75 & 17,05 & \\
\hline \multirow{2}{*}{$\begin{array}{l}\text { Verzögerte Wiedergabe } \\
\text { WMS-R }\end{array}$} & Gesunde VPn & 8 & 102,25 & 14,41 & \multirow{2}{*}{,612 } \\
\hline & PD-Patienten & 8 & 106,12 & 15,40 & \\
\hline
\end{tabular}

$\mathrm{N}=$ Anzahl der Probanden; MW=Mittelwert; SD=Standardabweichung 


\subsubsection{Gedächtnisleistungen in der 2. Untersuchung}

Nach Beendigung der Therapie bzw. in der 2. neuropsychologischen Untersuchung konnten teilweise tendenzielle, jedoch nicht signifikante Verbesserungen der einzelnen Gedächtnisleistungen (verbale und non-verbale Merkspanne, verbales und non-verbales Kurzzeitgedächtnis, allgemeines und Langzeitgedächtnis) bei den PD-Patienten der Therapiebedingung im Vergleich zu den PD-Patienten der Kontrollbedingung beobachtet werden; (Tab. 21).

Tab.21 Mittelwerte der Gedächtnisleistungen PD-Patienten in Kontroll- und Therapiebedingung in der 2. neuropsychologischen Untersuchung, gemessen mit WMS-R-Subtests

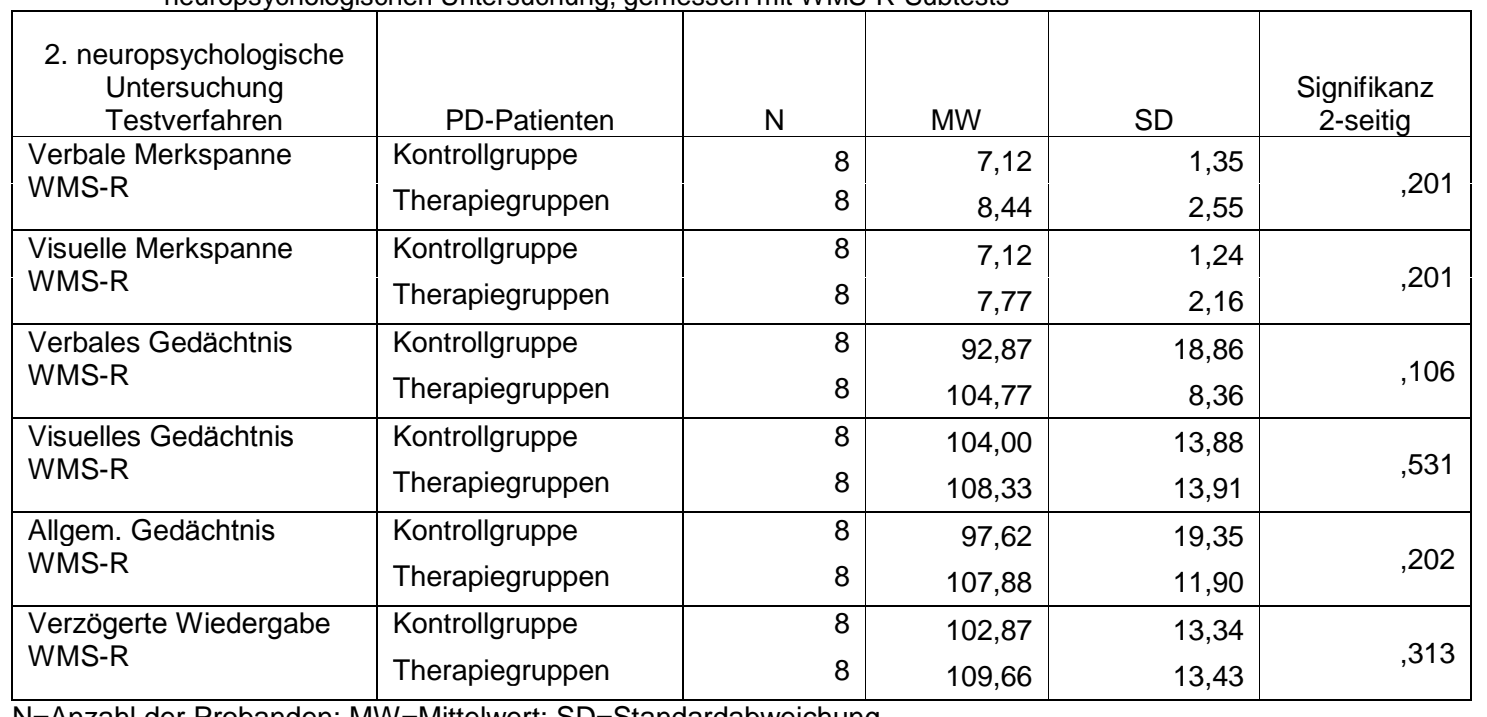

$\mathrm{N}=$ Anzahl der Probanden; MW=Mittelwert; SD=Standardabweichung

Auch die die gesunden VPN der Therapiegruppen erreichten im Vergleich zu den gesunden VPN der Kontrollbedingung tendenzielle, jedoch nicht signifikante Verbesserungen in sämtlichen untersuchten Gedächtnisleistungen (gemessen mit der WMS-R); (Tab. 22). 
Tab.22 Mittelwerte der Gedächtnisleistungen der gesunden VPN in Kontroll- und Therapiebedingung in der 2. neuropsychologischen Untersuchung, gemessen mit WMS-R-Subtests

\begin{tabular}{|c|c|c|c|c|c|}
\hline $\begin{array}{l}\text { 2. neuropsychologische } \\
\text { Untersuchung }\end{array}$ & Gesunde VPN & $\mathrm{N}$ & MW & SD & $\begin{array}{l}\text { Signifikanz } \\
\text { 2-seitig }\end{array}$ \\
\hline \multirow{2}{*}{$\begin{array}{l}\text { Verbale Merkspanne } \\
\text { WMS-R }\end{array}$} & Kontrollgruppe & 8 & 6,87 & 1,12 & \multirow{2}{*}{,246 } \\
\hline & Therapiegruppen & 8 & 7,85 & 1,95 & \\
\hline \multirow{2}{*}{$\begin{array}{l}\text { Visuelle Merkspanne } \\
\text { WMS-R }\end{array}$} & Kontrollgruppe & 8 & 7,37 & 1,06 & \multirow{2}{*}{,229 } \\
\hline & Therapiegruppen & 8 & 8,00 & ,81 & \\
\hline \multirow{2}{*}{$\begin{array}{l}\text { Verbales Gedächtnis } \\
\text { WMS-R }\end{array}$} & Kontrollgruppe & 8 & 102,62 & 25,60 & \multirow{2}{*}{,481 } \\
\hline & Therapiegruppen & 8 & 111,00 & 17,70 & \\
\hline \multirow{2}{*}{$\begin{array}{l}\text { Visuelles Gedächtnis } \\
\text { WMS-R }\end{array}$} & Kontrollgruppe & 8 & 105,50 & 13,98 & \multirow{2}{*}{,629 } \\
\hline & Therapiegruppen & 8 & 109,00 & 13,29 & \\
\hline \multirow{2}{*}{$\begin{array}{l}\text { Allgem. Gedächtnis } \\
\text { WMS-R }\end{array}$} & Kontrollgruppe & 8 & 105,62 & 23,56 & \multirow{2}{*}{,571 } \\
\hline & Therapiegruppen & 8 & 111,71 & 15,54 & \\
\hline \multirow{2}{*}{$\begin{array}{l}\text { Verzögerte Wiedergabe } \\
\text { WMS-R }\end{array}$} & Kontrollgruppe & 8 & 107,25 & 15,11 & \multirow{2}{*}{,270 } \\
\hline & Therapiegruppen & 8 & 116,28 & 15,21 & \\
\hline
\end{tabular}

$\mathrm{N}=$ Anzahl der Probanden; MW=Mittelwert; SD=Standardabweichung

Die gesunden VPN und die PD-Patienten in der Therapiebedingung unterschieden sich in der 2. neuropsychologischen Untersuchung im Hinblick auf ihre verbale und non-verbale Merkspanne, das verbale und non-verbale Kurzzeitgedächtnis sowie das allgemeine Gedächtnis und das Langzeitgedächtnis, gemessen mit der Wechsler-Memory-Scale-R, nicht signifikant voneinander; (Tab. 23).

Tab.23 Mittelwerte der Gedächtnisleistungen der PD-Patienten und der gesunden VPN in Therapiebedingung in der 2. neuropsychologischen Untersuchung, gemessen mit WMS-R-Subtests

\begin{tabular}{|c|c|c|c|c|c|}
\hline $\begin{array}{l}\text { 2. neuropsychologische } \\
\text { Untersuchung } \\
\text { Testverfahren }\end{array}$ & $\begin{array}{c}\text { VPN } \\
\text { Therapiegruppen }\end{array}$ & $\mathrm{N}$ & MW & SD & $\begin{array}{l}\text { Signifikanz } \\
\text { 2-seitig }\end{array}$ \\
\hline \multirow{2}{*}{$\begin{array}{l}\text { Verbale Merkspanne } \\
\text { WMS-R }\end{array}$} & Gesunde VPN & 8 & 7,85 & 1,95 & \multirow{2}{*}{,623 } \\
\hline & PD-Patienten & 8 & 8,44 & 2,55 & \\
\hline \multirow{2}{*}{$\begin{array}{l}\text { Visuelle Merkspanne } \\
\text { WMS-R }\end{array}$} & Gesunde VPN & 8 & 8,00 & 81 & \multirow{2}{*}{,783 } \\
\hline & PD-Patienten & 8 & 7,77 & 2,16 & \\
\hline \multirow{2}{*}{$\begin{array}{l}\text { Verbales Gedächtnis } \\
\text { WMS-R }\end{array}$} & Gesunde VPN & 8 & 111,00 & 17,70 & \multirow{2}{*}{,415 } \\
\hline & PD-Patienten & 8 & 104,77 & 8,36 & \\
\hline \multirow{2}{*}{$\begin{array}{l}\text { Visuelles Gedächtnis } \\
\text { WMS-R }\end{array}$} & Gesunde VPN & 8 & 109,00 & 13,29 & \multirow{2}{*}{,924 } \\
\hline & PD-Patienten & 8 & 108,33 & 13,91 & \\
\hline \multirow{2}{*}{$\begin{array}{l}\text { Allgem. Gedächtnis } \\
\text { WMS-R }\end{array}$} & Gesunde VPN & 8 & 111,71 & 15,54 & \multirow{2}{*}{, 585} \\
\hline & PD-Patienten & 8 & 107,88 & 11,90 & \\
\hline \multirow{2}{*}{$\begin{array}{l}\text { Verzögerte Wiedergabe } \\
\text { WMS-R }\end{array}$} & Gesunde VPN & 8 & 116,28 & 15,21 & \multirow{2}{*}{,372 } \\
\hline & PD-Patienten & 8 & 109,66 & 13,43 & \\
\hline
\end{tabular}

$\mathrm{N}=$ Anzahl der Probanden; MW=Mittelwert; SD=Standardabweichung 
Die PD-Patienten der Kontrollbedingung zeigten im Vergleich zu den gesunden VPN der Gruppe tendenziell eingeschränktere Gedächtnisleistungen (verbale und non-verbale Merkspanne und Kurzzeitgedächtnis sowie allgemeines und Langzeitgedächtnis); (Tab. 24).

Tab.24 Mittelwerte der Gedächtnisleistungen der PD-Patienten und der gesunden VPN in Kontrollbedingung in der 2. neuropsychologischen Untersuchung, gemessen mit WMS-R-Subtests

\begin{tabular}{|c|c|c|c|c|c|}
\hline $\begin{array}{l}\text { 2. neuropsychologische } \\
\text { Untersuchung } \\
\text { Testverfahren }\end{array}$ & $\begin{array}{c}\text { VPN } \\
\text { Kontrollgruppe }\end{array}$ & $\mathrm{N}$ & MW & SD & $\begin{array}{l}\text { Signifikanz } \\
\text { 2-seitig }\end{array}$ \\
\hline \multirow{2}{*}{$\begin{array}{l}\text { Verbale Merkspanne } \\
\text { WMS-R }\end{array}$} & Gesunde VPN & 8 & 6,87 & 1,12 & \multirow{2}{*}{,694 } \\
\hline & PD-Patienten & 8 & 7,12 & 1,35 & \\
\hline \multirow{2}{*}{$\begin{array}{l}\text { Visuelle Merkspanne } \\
\text { WMS-R }\end{array}$} & Gesunde VPN & 8 & 7,37 & 1,06 & \multirow{2}{*}{,672 } \\
\hline & PD-Patienten & 8 & 7,12 & 1,24 & \\
\hline \multirow{2}{*}{$\begin{array}{l}\text { Verbales Gedächtnis } \\
\text { WMS-R }\end{array}$} & Gesunde VPN & 8 & 102,62 & 25,60 & \multirow[t]{2}{*}{,400 } \\
\hline & PD-Patienten & 8 & 92,87 & 18,86 & \\
\hline \multirow{2}{*}{$\begin{array}{l}\text { Visuelles Gedächtnis } \\
\text { WMS } 3\end{array}$} & Gesunde VPN & 8 & 105,50 & 13,98 & \multirow{2}{*}{,833 } \\
\hline & PD-Patienten & 8 & 104,00 & 13,88 & \\
\hline \multirow{2}{*}{$\begin{array}{l}\text { Allgem. Gedächtnis } \\
\text { WMS-R }\end{array}$} & Gesunde VPN & 8 & 105,62 & 23,56 & \multirow{2}{*}{,470 } \\
\hline & PD-Patienten & 8 & 97,62 & 19,35 & \\
\hline \multirow{2}{*}{$\begin{array}{l}\text { Verzögerte Wiedergabe } \\
\text { WMS-R }\end{array}$} & Gesunde VPN & 8 & 107,25 & 15,11 & \multirow{2}{*}{, 549} \\
\hline & PD-Patienten & 8 & 102,87 & 13,34 & \\
\hline
\end{tabular}

$\mathrm{N}=$ Anzahl der Probanden; MW=Mittelwert; SD=Standardabweichung

\subsubsection{Vergleich der Gedächtnisleistungen in der 1. und 2. Untersuchung}

In der Therapiegruppe konnten sich die Patienten insbesondere hinsichtlich der Kapazität des visuellen Kurzzeitgedächtnisses (WMS-R Subtests) $(\mathrm{MW}$ vorher = 95,11 Punkte, SD=9,87 Punkte; MW nachher $=108,33$ Punkte, $S D=13,91$ Punkte; $p<0,01$ ) und des allgemeinen Gedächtnisses (MW vorher $=94,66$ Punkte, SD= 13,41 Punkte; MW nachher $=107,88$ Punkte, SD = 11,90 Punkte; $\mathrm{p}<.05)$ signifikant verbessern; (Abb.10, 11; Tab. 25). 


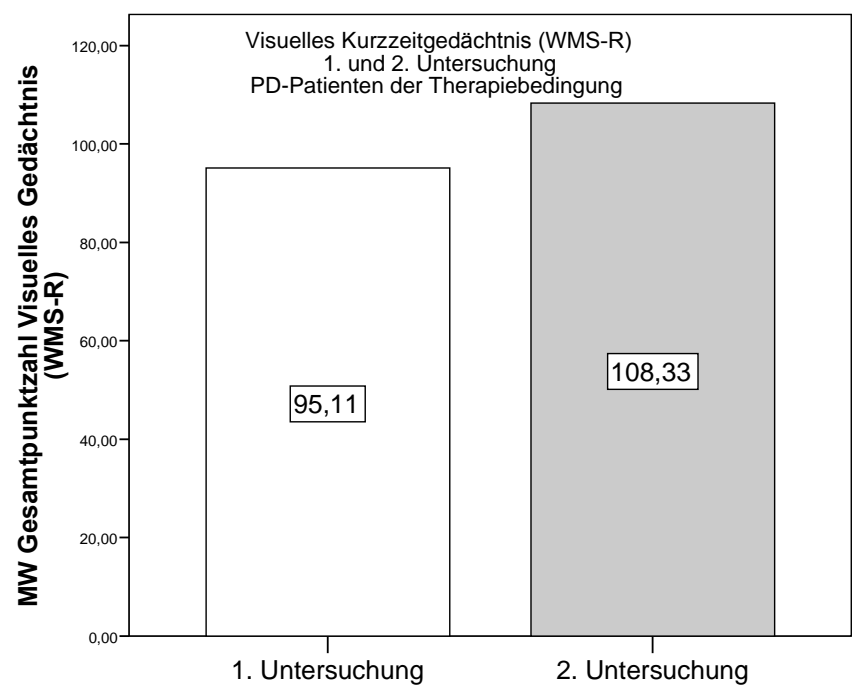

Abb.10 Visuelles Kurzzeitgedächtnis der PD-Patienten in Therapiebedingung in der 1 . und 2 . Untersuchung $(p=0,007)$

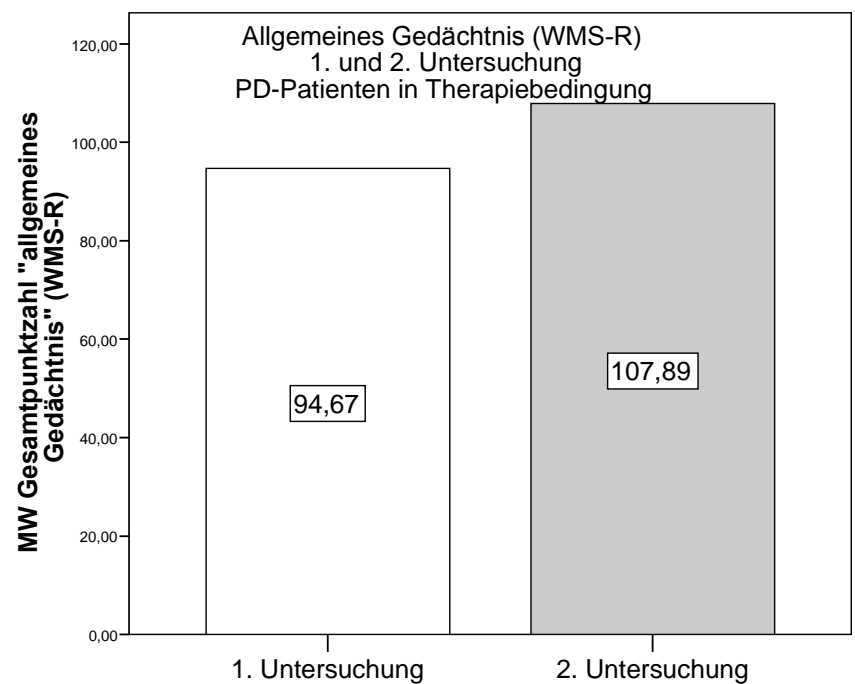

Abb.11 Allgemeines Gedächtnis der PD-Patienten in Therapiebedingung in der 1. und 2. Untersuchung $(p=0,016)$ 
Alle weiteren untersuchten Gedächtnisbereiche, gemessen mit Subtests aus WMS-R, wie verbale Merkspanne und Kurzzeitgedächtnis, visuelle Merkspanne sowie das modalitätsübergreifende Langzeitgedächtnis konnten tendenziell verbessert werden; (Tab. 25).

Tab.25 Mittelwerte der Gedächtnisleistungen der PD-Patienten in Therapiebedingung in der 1 . und 2 neuropsychologischen Untersuchung, gemessen mit WMS-R-Subtests

\begin{tabular}{|c|c|c|c|c|}
\hline $\begin{array}{l}\text { 1. und 2. neuropsychologische Untersuchung } \\
\text { Testverfahren }\end{array}$ & $\begin{array}{l}\text { PD-Patienten } \\
\text { Therapiegruppen } \\
\mathrm{N}\end{array}$ & MW & SD & $\begin{array}{l}\text { Signifikanz } \\
\text { 2-seitig }\end{array}$ \\
\hline $\begin{array}{l}\text { Verbale Merkspanne (WMS-R) vorher } \\
\text { Verbale Merkspanne (WMS-R) nachher }\end{array}$ & 8 & $\begin{array}{l}7,88 \\
8,44\end{array}$ & $\begin{array}{l}1,76 \\
2,55\end{array}$ & 214 \\
\hline $\begin{array}{l}\text { Verbales Kurzzeitgedächtnis (WMS-R) } \\
\text { Vorher } \\
\text { Verbales Kurzzeitgedächtnis (WMS-R) } \\
\text { Nachher }\end{array}$ & 8 & 94,66 & $\begin{array}{r}15,19 \\
8,36\end{array}$ & ,075 \\
\hline $\begin{array}{l}\text { Visuelle Merkspanne (WMS-R) vorher } \\
\text { Visuelle Merkspanne (WMS-R) nachher }\end{array}$ & 8 & $\begin{array}{l}6,88 \\
7,77\end{array}$ & $\begin{array}{l}1,05 \\
2,16\end{array}$ & 272 \\
\hline $\begin{array}{l}\text { Visuelles Kurzzeitgedächtnis (WMS-R) } \\
\text { Vorher } \\
\text { Visuelles Kurzzeitgedächtnis (WMS-R) } \\
\text { Nachher }\end{array}$ & 8 & 95,11 & 9,87 & ,007, \\
\hline $\begin{array}{l}\text { Verbales u. visuelles Langzeitgedächtnis } \\
\text { (WMS-R) vorher } \\
\text { Verbales u. visuelles Langzeitgedächtnis } \\
\text { (WMS-R) nachher }\end{array}$ & 8 & 101,55 & 10,84 & 120, \\
\hline $\begin{array}{l}\text { Allgem. Gedächtnis WMS-R vorher } \\
\text { Allgem. Gedächtnis WMS-R nachher }\end{array}$ & 8 & $\begin{array}{r}94,66 \\
107,88\end{array}$ & $\begin{array}{l}13,41 \\
11,90\end{array}$ & ,016, \\
\hline $\begin{array}{l}\text { Verzögerte Wiedergabe (WMS-R ) vorher } \\
\text { Verzögerte Wiedergabe (WMS-R) nachher }\end{array}$ & 8 & $\begin{array}{l}101.55 \\
109,66\end{array}$ & $\begin{array}{l}10,84 \\
14,43\end{array}$ & 0,120 \\
\hline
\end{tabular}

$\mathrm{N}=$ Anzahl der Probanden; MW=Mittelwert; SD=Standardabweichung

Bei den PD-Patienten in der Kontrollbedingung zeigte sich in der 2. Untersuchung nach 4 Monaten im Vergleich zu den zuvor genannten Verbesserungen der PD-Patienten der Therapiegruppen ein tendenziell gegenteiliger Effekt im Bereich der untersuchten Gedächtnisleistungen (Tab. 26). 
Tab.26 Mittelwerte der Gedächtnisleistungen der PD-Patienten in Kontrollbedingung in der 1. und 2. neuropsychologischen Untersuchung, gemessen mit WMS-R-Subtests

\begin{tabular}{|c|c|c|c|c|}
\hline $\begin{array}{l}\text { 1. und 2. neuropsychologische Untersuchung } \\
\text { Testverfahren }\end{array}$ & $\begin{array}{c}\text { PD-Patienten } \\
\text { Kontrollgruppe } \\
\mathrm{N}\end{array}$ & MW & SD & $\begin{array}{l}\text { Signifikanz } \\
\text { 2-seitig }\end{array}$ \\
\hline $\begin{array}{l}\text { Verbale Merkspanne (WMS-R) } \\
\text { Vorher } \\
\text { Verbale Merkspanne (WMS-R) } \\
\text { Nachher }\end{array}$ & 8 & 7,62 & $\begin{array}{l}1,76 \\
1,35\end{array}$ & 516, \\
\hline $\begin{array}{l}\text { Verbales Kurzzeitgedächtnis (WMS-R) } \\
\text { Vorher } \\
\text { Verbales Kurzzeitgedächtnis (WMS-R) } \\
\text { Nachher }\end{array}$ & 8 & 96,75 & $\begin{array}{l}17,87 \\
18,86\end{array}$ & 419, \\
\hline $\begin{array}{l}\text { Visuelle Merkspanne (WMS-R) } \\
\text { Vorher } \\
\text { Visuelle Merkspanne (WMS-R) } \\
\text { Nachher }\end{array}$ & 8 & 7,37 & ,91 & 598, \\
\hline $\begin{array}{l}\text { Visuelles Kurzzeitgedächtnis (WMS-R) } \\
\text { Vorher } \\
\text { Visuelles Kurzzeitgedächtnis( WMS-R) } \\
\text { Nachher }\end{array}$ & 8 & 102,00 & $\begin{array}{l}19,75 \\
13,88\end{array}$ & ,718 \\
\hline $\begin{array}{l}\text { Verbales und visuelles Langzeitgedächtnis } \\
\text { (WMS-R) vorher } \\
\text { Verbales und visuelles Langzeitgedächtnis } \\
\text { (WMS-R) nachher }\end{array}$ & 8 & 106,12 & 15,40 & 445 \\
\hline $\begin{array}{l}\text { Allgem. Gedächtnis (WMS-R) vorher } \\
\text { Allgem. Gedächtnis (WMS-R) nachher }\end{array}$ & 8 & $\begin{array}{l}98,75 \\
97,62\end{array}$ & $\begin{array}{l}17,05 \\
19,35\end{array}$ & ,759 \\
\hline $\begin{array}{l}\text { Verzögerte Wiedergabe (WMS-R) vorher } \\
\text { Verzögerte Wiedergabe (WMS-R) nachher }\end{array}$ & 8 & $\begin{array}{l}106,12 \\
102,87\end{array}$ & $\begin{array}{l}15,40 \\
13,34\end{array}$ & 0,445 \\
\hline
\end{tabular}

$\mathrm{N}=$ Anzahl der Probanden; $\mathrm{MW}=$ Mittelwert; $\mathrm{SD}=$ Standardabweichung

Die gesunden Probanden in Therapiebedingung konnten die Kapazität ihres verbalen Kurzzeitgedächtnisses nach Beendigung der Therapie deutlich verbessern. Die Leistung des allgemeinen Kurzzeitgedächtnisses, gemessen mit der Wechsler-Memory-Scale-R (WMS-R Subtest „Allgemeines Gedächtnis“) ergab in der 1. Untersuchung für die gesunden Probanden der Therapiebedingung einen Mittelwert (MW) von 100,57 Punkten mit einer Standardabweichung (SD) von 12,24 Punkten und in der 2. Untersuchung einen signifikant besseren MW von 111,71 Punkten, SD=15,54 ( $p<0.05$ ); (Abb.12; Tab. 27). 


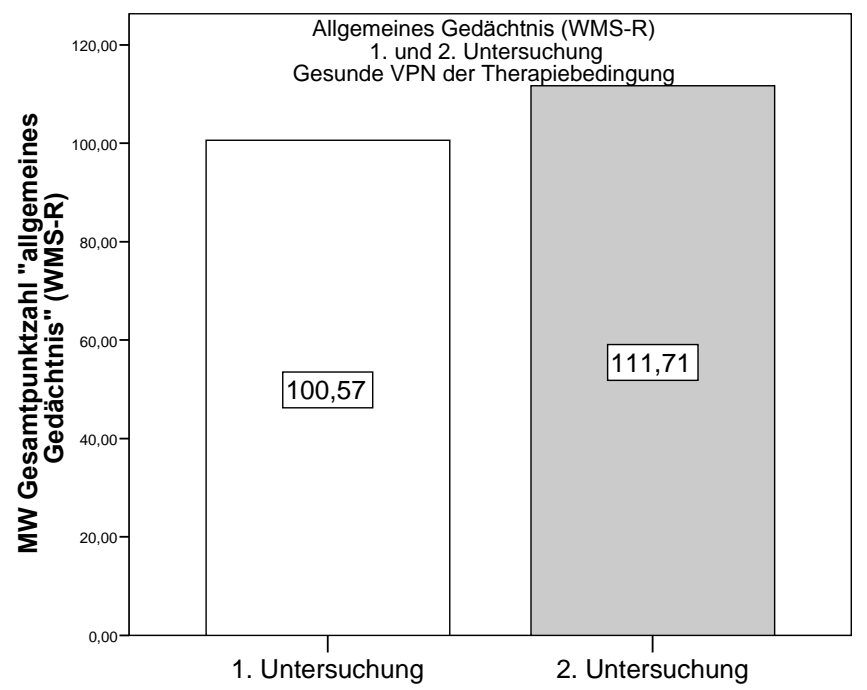

Abb.12 Allgemeines Gedächtnis der gesunden VPN in Therapiebedingung in der 1. und 2. Untersuchung $(p=0,039)$

Auch im Bereich des allgemeinen Langzeitgedächtnisses zeigte sich nach 4 Monaten ein signifikanter Unterschied, wobei die Leistung des allgemeinen Langzeitgedächtnisses in der 1. Untersuchung für die gesunden Probanden der Therapiebedingung mit einem Mittelwert (MW) von 106 Punkten und einer Standardabweichung (SD) von 11,59 Punkten signifikant schlechter war als die Leistung der 2. Untersuchung mit einem MW von 116,28 Punkten, SD=15,21 (p<.05); (Abb. 13; Tab. 27). 


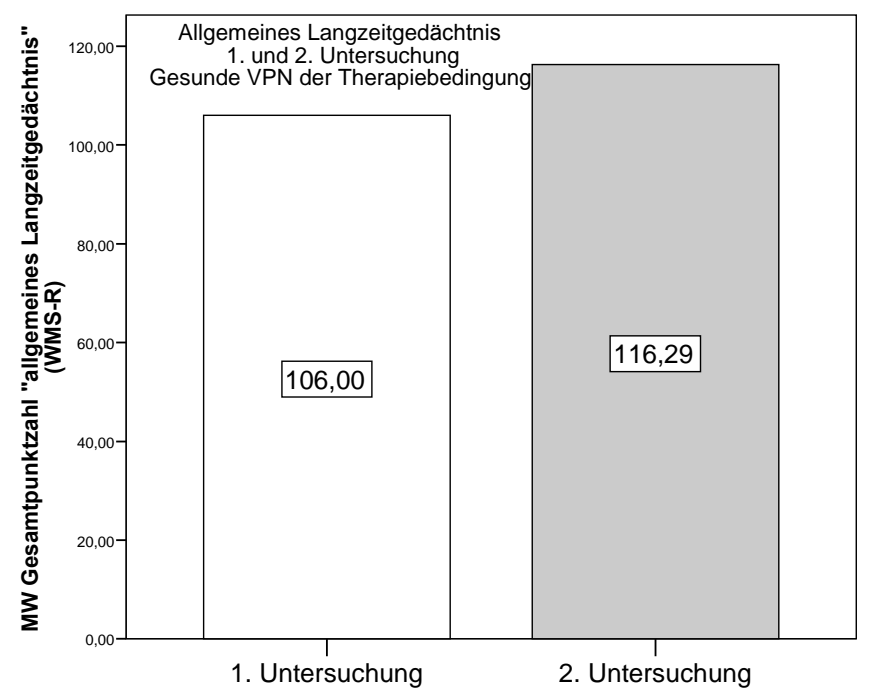

Abb.13 Allgemeines Langzeitgedächtnis der gesunden VPN in Therapiebedingung in der 1 . und 2 . Untersuchung $(p=0,039)$

Die weiteren Vergleiche der Ergebnisse der gesunden VPN in Therapiebedingung zwischen der 1. und 2. neuropsychologischen Untersuchung im Hinblick auf die verbale Merkspanne und der non-verbalen Merkspanne, ergab zwar tendenzielle Verbesserungen, jedoch keine signifikanten Unterschiede. Auch die Leistungsfähigkeit des verbalen und visuellen Kurzzeitgedächtnisses konnte tendenziell, aber nicht signifikant verbessert werden; (Tab. 27). 
Tab.27 Mittelwerte der Gedächtnisleistungen der gesunden VPN in Therapiebedingung in der 1. und 2. neuropsychologischen Untersuchung, gemessen mit WMS-R-Subtests

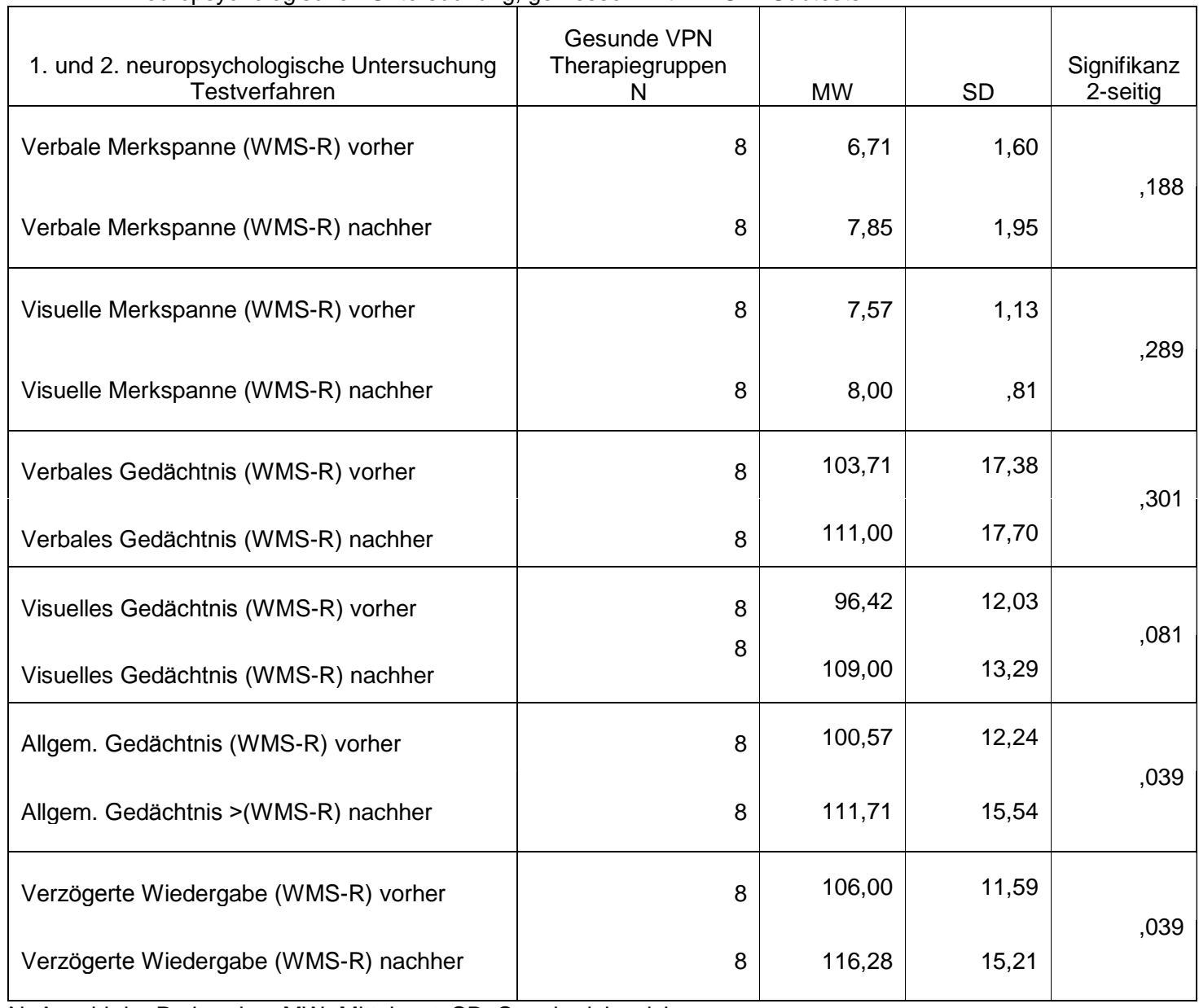

$\mathrm{N}=$ Anzahl der Probanden; $\mathrm{MW}=$ Mittelwert; $\mathrm{SD}=$ Standardabweichung

Die gesunden VPN der Kontrollbedingung zeigten nach vier Monaten keine Leistungsveränderungen bzgl. der modalitätsübergreifenden Merkspanne sowie des Kurz- und Langzeitgedächtnisses; (Tab. 28). 
Tab.28 Mittelwerte der Gedächtnisleistungen der gesunden VPN in Kontrollbedingung in der 1. und 2. neuropsychologischen Untersuchung, gemessen mit WMS-R-Subtests

\begin{tabular}{|c|c|c|c|c|}
\hline $\begin{array}{l}\text { 1. und 2. neuropsychologische Untersuchung } \\
\text { Testverfahren }\end{array}$ & $\begin{array}{c}\text { Gesunde VPN } \\
\text { Kontrollgruppe } \\
\text { N }\end{array}$ & MW & SD & $\begin{array}{l}\text { Signifikanz } \\
\text { 2-seitig }\end{array}$ \\
\hline $\begin{array}{l}\text { Verbale Merkspanne (WMS-R) vorher } \\
\text { Verbale Merkspanne (WMS-R) nachher }\end{array}$ & 8 & $\begin{array}{l}7,87 \\
6,87\end{array}$ & $\begin{array}{l}1,88 \\
1,12\end{array}$ & 155, \\
\hline $\begin{array}{l}\text { Visuelle Merkspanne (WMS-R) vorher } \\
\text { Visuelle Merkspanne (WMS-R) nachher }\end{array}$ & 8 & $\begin{array}{l}6,87 \\
7,37\end{array}$ & $\begin{array}{l}1,88 \\
1,06\end{array}$ & 381 \\
\hline $\begin{array}{l}\text { Verbales Gedächtnis (WMS-R) vorher } \\
\text { Verbales Gedächtnis (WMS-R) nachher }\end{array}$ & 8 & $\begin{array}{r}94,37 \\
102,62\end{array}$ & $\begin{array}{l}13,79 \\
25,60\end{array}$ & ,289 \\
\hline $\begin{array}{l}\text { Visuelles Gedächtnis (WMS-R) vorher } \\
\text { Visuelles Gedächtnis (WMS-R) nachher }\end{array}$ & 8 & $\begin{array}{r}99,25 \\
105,50\end{array}$ & $\begin{array}{l}16,73 \\
13,98\end{array}$ & ,064 \\
\hline $\begin{array}{l}\text { Allgem. Gedächtnis (WMS-R) vorher } \\
\text { Allgem. Gedächtnis (WMS-R) nachher }\end{array}$ & 8 & $\begin{array}{r}96,25 \\
105,62\end{array}$ & $\begin{array}{l}15,83 \\
23,56\end{array}$ & 199 \\
\hline $\begin{array}{l}\text { Verzögerte Wiedergabe (WMS-R) vorher } \\
\text { Verzögerte Wiedergabe (WMS-R) nachher }\end{array}$ & 8 & $\begin{array}{l}102,25 \\
107,25\end{array}$ & $\begin{array}{l}14,41 \\
15,11\end{array}$ & ,118 \\
\hline
\end{tabular}

$\mathrm{N}=$ Anzahl der Probanden; $\mathrm{MW}=$ Mittelwert; $\mathrm{SD}=$ Standardabweichung

\subsection{Exekutive Funktionen}

\subsubsection{Leistungsfähigkeit im Bereich der exekutiven Funktionen in der 1. Untersuchung}

In der Kapazität des visuellen Arbeitsgedächtnisses, gemessen mit der Wechsler-Memory-Scale-R (WMS-R Subtest „Blockspanne rückwärts“) unterschieden sich die PD-Patienten der Therapie- und der Kontrollbedingung signifikant voneinander $(\mathrm{p}<.05)$, wobei die Patienten in der Therapiebedingung eine schlechtere Leistung mit einem niedrigeren Mittelwert (MW) von 6,11 Punkten mit einer Standardabweichung (SD) von 1,05 Punkten erzielten. Die Kontrollgruppe erreichte hier einen Mittelwert (MW) von 8,0 Punkten mit einer Standardabweichung (SD) von 1,85 Punkten. Die Kapazität des verbalen 
Arbeitsgedächtnisses, der kognitiven Flexibilität und des problemlösenden Handelns stellte sich in der 1. Untersuchung in beiden Gruppen als nicht signifikant unterschiedlich dar; (Tab. 29).

Tab.29 Mittelwerte der exekutiven Funktionen der PD-Patienten der Therapie- und Kontrollgruppen, gemessen mit WMS-R, TMT-B, TvH in der 1. neuropsychologischen Untersuchung

\begin{tabular}{|c|c|c|c|c|c|}
\hline $\begin{array}{c}\text { 1.neuropsychologische } \\
\text { Untersuchung } \\
\text { Testverfahren }\end{array}$ & $\begin{array}{l}\text { PD-Patienten } \\
\text { Therapiegruppen }\end{array}$ & $\mathrm{N}$ & MW & SD & $\begin{array}{l}\text { Signifikanz } \\
\text { 2-seitig }\end{array}$ \\
\hline \multirow{2}{*}{$\begin{array}{l}\text { Verbales Arbeitsgedächtnis } \\
\text { (WMS-R) }\end{array}$} & Kontrollgruppe & 8 & 5,87 & 1,24 & \multirow{2}{*}{,850 } \\
\hline & Therapiegruppen & 8 & 6,00 & 1,41 & \\
\hline \multirow{2}{*}{$\begin{array}{l}\text { Visuelles Arbeitsgedächtnis } \\
\text { (WMS-R) }\end{array}$} & Kontrollgruppe & 8 & 8,00 & 1,85 & \multirow{2}{*}{019} \\
\hline & Therapiegruppen & 8 & 6,11 & 1,05 & \\
\hline \multirow[t]{2}{*}{ Trail-Making-Test-B (TMT-B) } & Kontrollgruppe & 8 & 168,00 & 93,85 & \multirow{2}{*}{,445 } \\
\hline & Therapiegruppen & 8 & 141,44 & 37,46 & \\
\hline \multirow[t]{2}{*}{ Turm von Hanoi (TvH) } & Kontrollgruppe & 8 & 12,00 & 5,78 & \multirow{2}{*}{,881 } \\
\hline & Therapiegruppen & 8 & 12,44 & 6,20 & \\
\hline
\end{tabular}

$\mathrm{N}=$ Anzahl der Probanden; MW=Mittelwert; SD=Standardabweichung

In der 1. neuropsychologischen Untersuchung unterschieden sich die gesunden VPN der Therapie- und Kontrollbedingung in den exekutiven Funktionen nicht signifikant voneinander; (Tab. 30).

Tab.30 Mittelwerte der exekutiven Funktionen der gesunden VPN der Therapie- und Kontrollgruppen, gemessen mit WMS-R, TMT-B, TvH in der 1. neuropsychologischen Untersuchung

\begin{tabular}{|c|c|c|c|c|c|}
\hline $\begin{array}{l}\text { 1.neuropsychologische } \\
\text { Untersuchung } \\
\text { Testverfahren }\end{array}$ & Gesunde VPN & $\mathrm{N}$ & MW & SD & $\begin{array}{c}\text { Signifikanz } \\
\text { 2-seitig }\end{array}$ \\
\hline \multirow{2}{*}{$\begin{array}{l}\text { Verbales Arbeitsgedächtnis } \\
\text { (WMS-R) }\end{array}$} & Kontrollgruppe & 8 & 6,50 & ,92 & \multirow{2}{*}{, 576} \\
\hline & Therapiegruppen & 8 & 6,14 & 1,46 & \\
\hline \multirow{2}{*}{$\begin{array}{l}\text { Visuelles Arbeitsgedächtnis } \\
\text { (WMS-R) }\end{array}$} & Kontrollgruppe & 8 & 6,37 & 1,92 & \multirow{2}{*}{,916 } \\
\hline & Therapiegruppen & 8 & 6,28 & 1,11 & \\
\hline \multirow{2}{*}{$\begin{array}{l}\text { Trail-Making-Test-B } \\
\text { (TMT-B) }\end{array}$} & Kontrollgruppe & 8 & 98,87 & 32,28 & \multirow{2}{*}{,255 } \\
\hline & Therapiegruppen & 8 & 117,71 & 28,46 & \\
\hline \multirow[t]{2}{*}{ Turm von Hanoi (TvH) } & Kontrollgruppe & 8 & 9,12 & 3,68 & \multirow{2}{*}{, 147 } \\
\hline & Therapiegruppen & 8 & 7,00 & 00 & \\
\hline
\end{tabular}

$\mathrm{N}=$ Anzahl der Probanden; MW=Mittelwert; SD=Standardabweichung

Im Bereich des problemlösenden Handelns, gemessen mit dem Turm-von-HanoiTest $(\mathrm{TvH})$ zeigte sich ein signifikanter Unterschied in der Leistungsfähigkeit zwischen den gesunden VPN und den PD-Patienten der Therapiegruppen 
$(p<.05)$, wobei die gesunden VPN in der Therapiebedingung einen Mittelwert (MW) von 7,00 Punkten mit einer Standardabweichung (SD) von 0 Punkten erzielten. Die PD-Patienten erreichten hier einen Mittelwert (MW) von 12,44 Punkten mit einer Standardabweichung (SD) von 6,20 Punkten (Abb.14;Tab. 31).

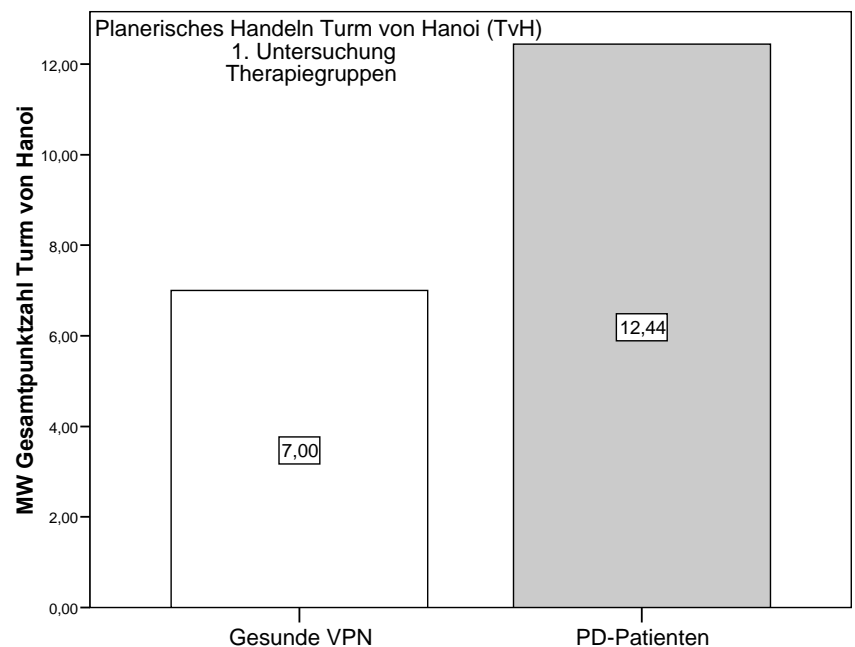

Abb.14 Fähigkeit des planerischen Handelns der PD-Patienten und der gesunden VPN in den Therapiegruppen in der 1 . Untersuchung $(p=0,037)$

Hinsichtlich des verbalen und des visuellen Arbeitsgedächtnisses sowie der kognitiven Flexibilität zeigten die PD-Patienten zwar insgesamt tendenziell, jedoch nicht signifikant schlechtere Leistungen; (Tab.31).

Tab.31 Mittelwerte der exekutiven Funktionen der PD-Patienten und der gesunden VPN in der Therapiebedingung, gemessen mit WMS-R, TMT-B, TvH in der 1 . neuropsychologischen Untersuchung

\begin{tabular}{|c|c|c|c|c|c|}
\hline $\begin{array}{l}\text { 1. neuropsychologische } \\
\text { Untersuchung } \\
\text { Testverfahren }\end{array}$ & $\begin{array}{c}\text { VPN } \\
\text { Therapiegruppen }\end{array}$ & $\mathrm{N}$ & MW & SD & $\begin{array}{l}\text { Signifikanz } \\
\text { 2-seitig }\end{array}$ \\
\hline \multirow{2}{*}{$\begin{array}{l}\text { Verbales Arbeitsgedächtnis } \\
\text { (WMS-R) }\end{array}$} & Gesunde VPN & 8 & 6,14 & 1,46 & \multirow{2}{*}{,846 } \\
\hline & PD-Patienten & 8 & 6,00 & 1,41 & \\
\hline \multirow{2}{*}{$\begin{array}{l}\text { Visuelles Arbeitsgedächtnis } \\
\text { (WMS-R) }\end{array}$} & Gesunde VPN & 8 & 6,28 & 1,11 & \multirow{2}{*}{, 753} \\
\hline & PD-Patienten & 8 & 6,11 & 1,05 & \\
\hline \multirow[t]{2}{*}{ Trail-Making-Test-B (TMT-B) } & Gesunde VPN & 8 & 117,71 & 28,46 & \multirow{2}{*}{,187 } \\
\hline & PD-Patienten & 8 & 141,44 & 37,46 & \\
\hline \multirow[t]{2}{*}{ Turm von Hanoi (TvH) } & Gesunde VPN & 8 & 7,00 & ,00 & \multirow{2}{*}{037} \\
\hline & PD-Patienten & 8 & 12,44 & 6,20 & \\
\hline
\end{tabular}

$\mathrm{N}=$ Anzahl der Probanden; MW=Mittelwert; SD=Standardabweichung 
Die gesunden VPN und die PD-Patienten der Kontrollgruppe zeigten in der 1. neuropsychologischen Untersuchung keine signitifikant unterschiedlichen Leistungen des Arbeitsgedächtnisses, der kognitiven Flexibilität und des planerischen Handelns; (Tab. 32).

Tab.32 Mittelwerte der exekutiven Funktionen der PD-Patienten und der gesunden VPN in der Kontrollbedingung, gemessen mit WMS-R, TMT-B, TvH in der 1. neuropsychologischen Untersuchung

\begin{tabular}{|c|c|c|c|c|c|}
\hline $\begin{array}{c}\text { 1. neuropsychologische } \\
\text { Untersuchung } \\
\text { Testverfahren }\end{array}$ & $\begin{array}{c}\text { VPN } \\
\text { Kontrollgruppe }\end{array}$ & $\mathrm{N}$ & MW & SD & $\begin{array}{l}\text { Signifikanz } \\
\text { 2-seitig }\end{array}$ \\
\hline $\begin{array}{l}\text { Verbales Arbeitsgedächtnis } \\
\text { (WMS-R) }\end{array}$ & $\begin{array}{l}\text { Gesunde VPN } \\
\text { PD-Patienten }\end{array}$ & $\begin{array}{l}8 \\
8\end{array}$ & $\begin{array}{l}6,50 \\
5,87\end{array}$ & $\begin{array}{r}, 92 \\
1,24\end{array}$ & ,274 \\
\hline $\begin{array}{l}\text { Visuelles Arbeitsgedächtnis } \\
\text { (WMS-R) }\end{array}$ & $\begin{array}{l}\text { Gesunde VPN } \\
\text { PD-Patienten }\end{array}$ & $\begin{array}{l}8 \\
8\end{array}$ & $\begin{array}{l}6,37 \\
8,00\end{array}$ & $\begin{array}{l}1,92 \\
1,85\end{array}$ & , 107 \\
\hline $\begin{array}{l}\text { Trail-Making-Test-B } \\
\text { (TMT-B) }\end{array}$ & $\begin{array}{l}\text { Gesunde VPN } \\
\text { PD-Patienten }\end{array}$ & $\begin{array}{l}8 \\
8\end{array}$ & $\begin{array}{r}98,87 \\
168,00\end{array}$ & $\begin{array}{l}32,28 \\
93,85\end{array}$ & ,069 \\
\hline Turm von Hanoi (TvH) & $\begin{array}{l}\text { Gesunde VPN } \\
\text { PD-Patienten }\end{array}$ & $\begin{array}{l}8 \\
8\end{array}$ & $\begin{array}{r}9,12 \\
12,00\end{array}$ & $\begin{array}{l}3,68 \\
5,78\end{array}$ & ,255 \\
\hline
\end{tabular}

$\mathrm{N}=$ Anzahl der Probanden; MW=Mittelwert; SD=Standardabweichung

\subsubsection{Leistungsfähigkeit im Bereich der exekutiven Funktionen in der 2. Untersuchung}

In der 2. neuropsychologischen Untersuchung unterschieden sich die PDPatienten der Therapie- und Kontrollbedingung im Hinblick auf das verbale Arbeitsgedächtnis signifikant voneinander $(p<.05)$, wobei die Patienten in der Kontrollbedingung einen Mittelwert (MW) von 4,75 Punkten mit einer Standardabweichung (SD) von 1,28 Punkten zeigten. Die Therapiegruppen erreichten hier einen Mittelwert (MW) von 6,55 Punkten mit einer Standardabweichung (SD) von 2,00 Punkten; (Abb.15;Tab.33). 


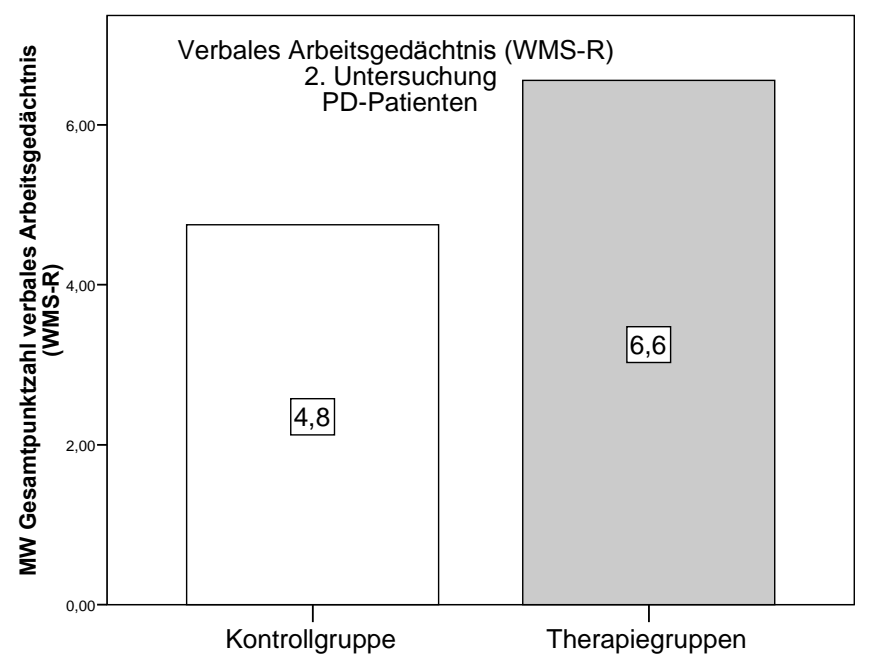

Abb.15 Verbales Arbeitsgedächtnis der PD-Patienten in den Therapie- und Kontrollgruppen in der 2.Untersuchung $(p=0,043)$

In allen weiteren Bereichen der exekutiven Funktionen konnte eine tendenzielle Leistungsverbesserung der PD-Patienten der Therapiegruppen im Vergleich zu den PD-Patienten der Kontrollgruppe festgestellt werden; (Tab. 33).

Tab.33 Mittelwerte der exekutiven Funktionen der PD-Patienten in den Therapie- und Kontrollbedingungen, gemessen mit WMS-R, TMT-B, TvH in der 2. neuropsychologischen Untersuchung

\begin{tabular}{|l|l|r|r|r|r|}
\hline \multicolumn{1}{|c}{$\begin{array}{c}\text { 2. neuropsychologische } \\
\text { Untersuchung } \\
\text { Testverfharen }\end{array}$} & PD-Patienten & N & MW & SD & \multicolumn{1}{c|}{$\begin{array}{c}\text { Signifikanz } \\
\text { 2-seitig }\end{array}$} \\
\hline $\begin{array}{l}\text { Verbales Arbeitsgedächtnis } \\
\text { (WMS-R) }\end{array}$ & $\begin{array}{l}\text { Kontrollgruppe } \\
\text { Therapiegruppen }\end{array}$ & 8 & 4,75 & 1,28 &, 043 \\
\hline $\begin{array}{l}\text { Visuelles Arbeitsgedächtnis } \\
\text { (WMS-R) }\end{array}$ & Kontrollgruppe & 8 & 6,55 & 2,00 &, 656 \\
& Therapiegruppen & 8 & 6,55 & 1,81 &, 263 \\
\hline $\begin{array}{l}\text { Trail-Making-Test-B } \\
\text { (TMT-B) }\end{array}$ & Kontrollgruppe & 8 & 181,00 & 109,91 & \\
& Therapiegruppen & 8 & 134,44 & 46,21 &, 869 \\
\hline Turm von Hanoi (TvH) & Kontrollgruppe & 8 & 8,25 & 3,15 & \\
& Therapiegruppen & 8 & 8,00 & 3,00 & \\
\hline
\end{tabular}

$\mathrm{N}=$ Anzahl der Probanden; $\mathrm{MW}=$ Mittelwert; $\mathrm{SD}=$ Standardabweichung

Auch die gesunden VPN der Therapie- und Kontrollbedingung zeigten einen signifikanten Leistungsunterschied im Hinblick auf das verbale Arbeitsgedächtnis 
$(p<.05)$, wobei die VPN in der Therapiebedingung einen höheren Mittelwert (MW) von 8,00 Punkten mit einer Standardabweichung (SD) von 2,16 Punkten zeigten. Die Kontrollgruppe erreichte hier nur einen Mittelwert (MW) von 5,75 Punkten mit einer Standardabweichung (SD) von 0,70 Punkten (Abb.16; Tab. 34).

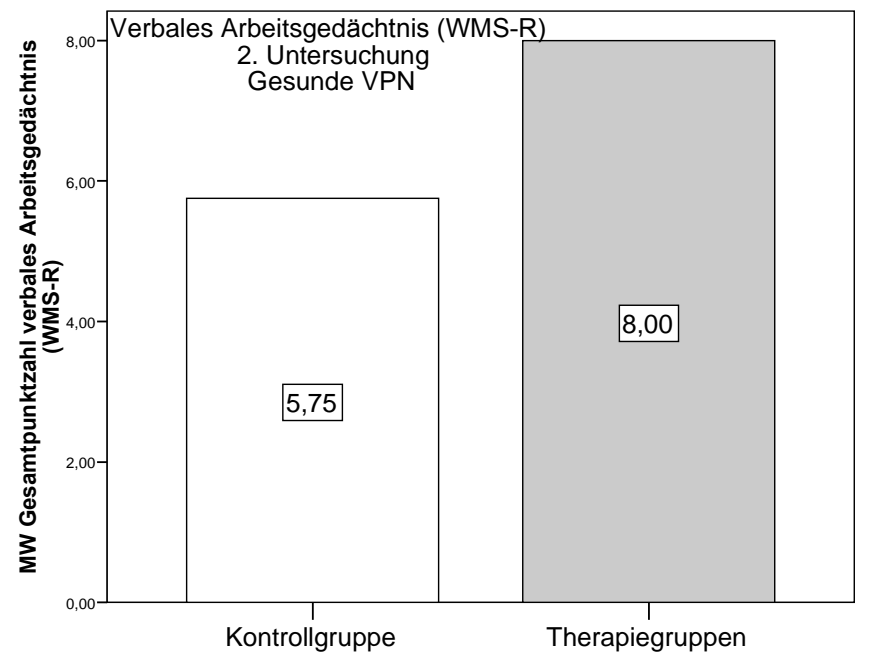

Abb.16 Verbales Arbeitsgedächtnis der gesunden VPN in den Therapie- und Kontrollgruppen in der 2. Untersuchung $(p=0,033)$

Im Bereich des visuellen Arbeitsgedächtnisses, der kognitiven Flexibilität und des planerischen Handelns zeigten die gesunden VPN der Therapie- und der Kontrollbedingung keine signifikanten Unterschiede (Tab. 34).

Tab.34 Mittelwerte der exekutiven Funktionen der gesunden VPN in den Therapie- und Kontrollbedingungen, gemessen mit WMS-R, TMT-B, TvH in der 2. neuropsychologischen Untersuchung

\begin{tabular}{|c|c|c|c|c|c|}
\hline $\begin{array}{c}\text { 2. neuropsychologische } \\
\text { Untersuchung } \\
\text { Testverfahren }\end{array}$ & Gesunde VPN & $\mathrm{N}$ & MW & SD & $\begin{array}{c}\text { Signifikanz } \\
\text { 2-seitig }\end{array}$ \\
\hline $\begin{array}{l}\text { Verbales Arbeitsgedächtnis } \\
\text { (WMS-R) }\end{array}$ & $\begin{array}{l}\text { Kontrollgruppe } \\
\text { Therapiegruppen }\end{array}$ & $\begin{array}{l}8 \\
8\end{array}$ & $\begin{array}{l}5,75 \\
8,00\end{array}$ & $\begin{array}{r}, 70 \\
2,16\end{array}$ & ,033 \\
\hline $\begin{array}{l}\text { Visuelles Arbeitsgedächtnis } \\
\text { (WMS-R) }\end{array}$ & $\begin{array}{l}\text { Kontrollgruppe } \\
\text { Therapiegruppen }\end{array}$ & $\begin{array}{l}8 \\
8\end{array}$ & $\begin{array}{l}6,62 \\
7,14\end{array}$ & $\begin{array}{l}, 91 \\
, 89\end{array}$ & ,291 \\
\hline $\begin{array}{l}\text { Trail-Making-Test-B } \\
\text { (TMT-B) }\end{array}$ & $\begin{array}{l}\text { Kontrollgruppe } \\
\text { Therapiegruppen }\end{array}$ & $\begin{array}{l}8 \\
8\end{array}$ & $\begin{array}{r}107,70 \\
83,71\end{array}$ & $\begin{array}{l}45,72 \\
21,92\end{array}$ & ,228 \\
\hline Turm von Hanoi (TvH) & $\begin{array}{l}\text { Kontrollgruppe } \\
\text { Therapiegruppen }\end{array}$ & $\begin{array}{l}8 \\
8\end{array}$ & $\begin{array}{l}8,87 \\
7,57\end{array}$ & $\begin{array}{r}2,74 \\
, 97\end{array}$ & ,242 \\
\hline
\end{tabular}

$\mathrm{N}=$ Anzahl der Probanden; MW=Mittelwert; SD=Standardabweichung 
Die Leistungen der PD-Patienten und der gesunden VPN der Therapiebedingung bzgl. des modalitätsübergreifenden Arbeitsgedächtnisses, der kognitiven Flexibilität und des planerischen Handelns unterschieden sich nicht signifikant voneinander; (Tab. 35).

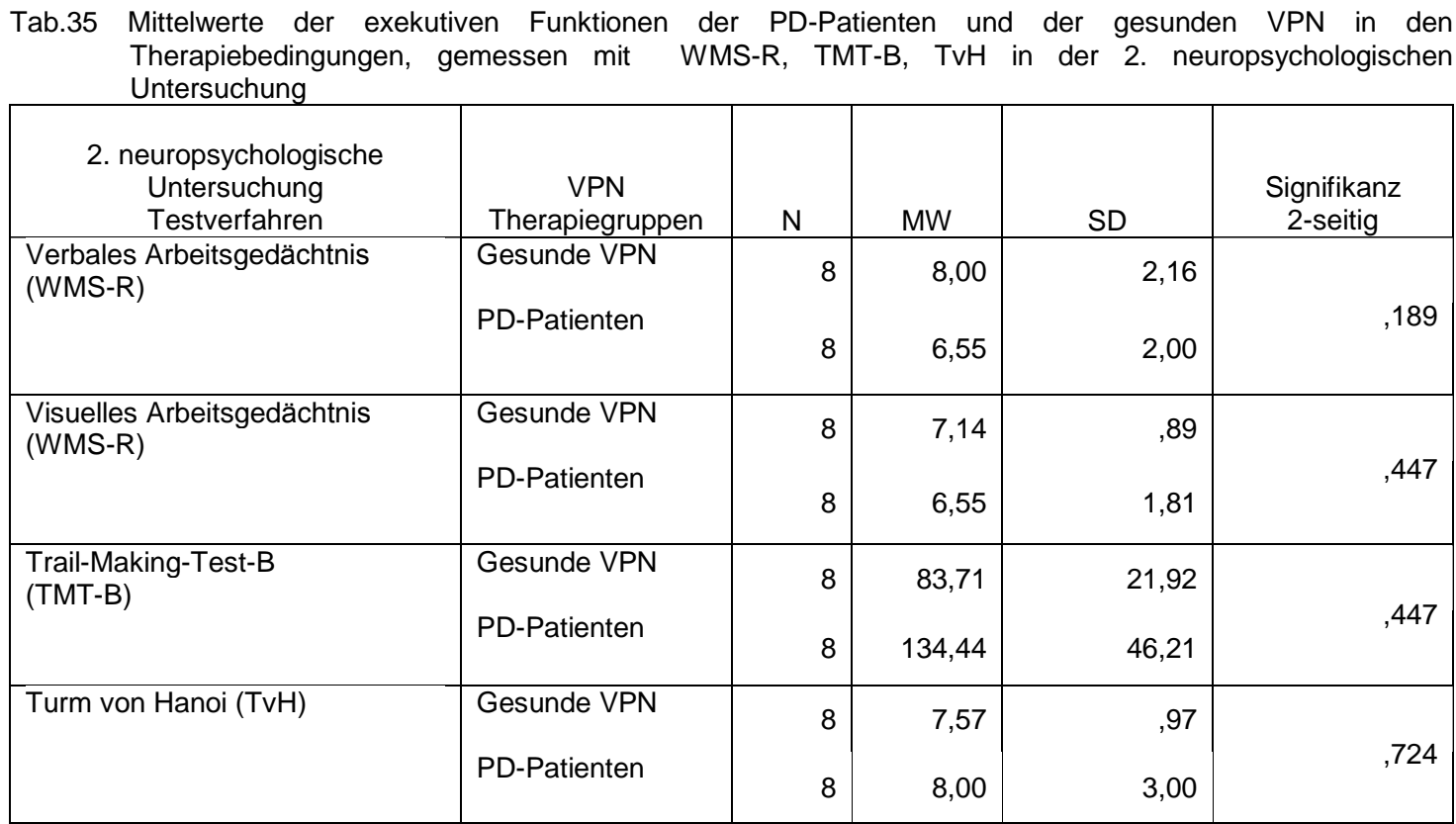

$\mathrm{N}=$ Anzahl der Probanden; $\mathrm{MW}=$ Mittelwert; $\mathrm{SD}=$ Standardabweichung

Der Vergleich zwischen den PD-Patienten und den gesunden VPN der Kontrollbedingung zeigte keine signifikant unterschiedliche Leistungsfähigkeit bezüglich des modalitätsübergreifenden Arbeitsgedächtnisses, der kognitiven Flexibilität und des planerischen Handelns; (Tab. 36). 
Tab.36 Mittelwerte der exekutiven Funktionen der PD-Patienten und der gesunden VPN in Kontrollbedingung, gemessen mit WMS-R, TMT-B, TVH in der 2. neuropsychologischen Untersuchung

\begin{tabular}{|c|c|c|c|c|c|}
\hline $\begin{array}{c}\text { 2. neuropsychologische } \\
\text { Untersuchung } \\
\text { Testverfahren }\end{array}$ & $\begin{array}{c}\text { VPN } \\
\text { Kontrollgruppe }\end{array}$ & $\mathrm{N}$ & MW & SD & $\begin{array}{l}\text { Signifikanz } \\
\text { 2-seitig }\end{array}$ \\
\hline \multirow{2}{*}{$\begin{array}{l}\text { Verbales Arbeitsgedächtnis } \\
\text { (WMS-R) }\end{array}$} & Gesunde VPN & 8 & 5,75 & 70 & \multirow{2}{*}{,074 } \\
\hline & PD-Patienten & 8 & 4,75 & 1,28 & \\
\hline \multirow{2}{*}{$\begin{array}{l}\text { Visuelles Arbeitsgedächtnis } \\
\text { (WMS-R) }\end{array}$} & Gesunde VPN & 8 & 6,62 & ,91 & \multirow[b]{2}{*}{, 547} \\
\hline & PD-Patienten & 8 & 6,12 & 2,10 & \\
\hline \multirow{2}{*}{$\begin{array}{l}\text { Trail-Making-Test-B } \\
\text { (TMT-B) }\end{array}$} & Gesunde VPN & 8 & 107,75 & 45,72 & \multirow{2}{*}{,115 } \\
\hline & PD-Patienten & 8 & 181,00 & 109,91 & \\
\hline \multirow[t]{2}{*}{ Turm von Hanoi (TvH) } & Gesunde VPN & 8 & 8,87 & 2,74 & \multirow[b]{2}{*}{679} \\
\hline & PD-Patienten & 8 & 8,25 & 3,15 & \\
\hline
\end{tabular}

$\mathrm{N}=$ Anzahl der Probanden; MW=Mittelwert; SD=Standardabweichung

\subsubsection{Vergleich der Leistungsfähigkeit im Bereich der exekutiven Funktionen in der 1. und 2. Untersuchung}

Die vor und nach Therapie durchgeführten Untersuchungen der Leistungsfähigkeit im Bereich der exekutiven Funktionen ergaben bei den PDPatienten in der Therapiebedingung eine insgesamt tendenzielle, jedoch nicht signifikante, Verbesserung des verbalen und visuellen Arbeitsgedächtnisses, der kognitiven Flexibilität und der Fähigkeit des planerischen Handelns; (Tab. 37).

Tab.37 Mittelwerte der exekutiven Funktionen der PD-Patienten in Therapiebedingung, gemessen mit WMS-R, TMT-B, TvH in der 1.und 2. neuropsychologischen Untersuchung

\begin{tabular}{|c|c|c|c|c|}
\hline $\begin{array}{l}\text { 1. und 2. neuropsychologische Untersuchung } \\
\text { Testverfahren }\end{array}$ & $\begin{array}{c}\text { PD-Patienten } \\
\text { Therapiegruppen } \\
\mathrm{N}\end{array}$ & MW & SD & $\begin{array}{l}\text { Signifikanz } \\
\text { 2-seitig }\end{array}$ \\
\hline $\begin{array}{l}\text { Verbales Arbeitsgedächtnis (WMS-R) } \\
\text { vorher } \\
\text { Verbales Arbeitsgedächtnis (WMS-R) } \\
\text { nachher }\end{array}$ & 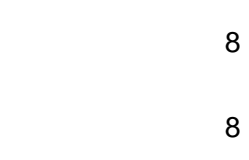 & 6,00 & 1,41 & 366, \\
\hline $\begin{array}{l}\text { Visuelles Arbeitsgedächtnis (WMS-R) } \\
\text { vorher } \\
\text { Visuelles Arbeitsgedächtnis (WMS-R) } \\
\text { nachher }\end{array}$ & 8 & 6,11 & 1,05 & 426, \\
\hline $\begin{array}{l}\text { Trail Making Test B (TMT-B) } \\
\text { vorher } \\
\text { Trail Making Test B (TMT-B) } \\
\text { nachher }\end{array}$ & 8 & $\begin{array}{l}141,44 \\
134,44\end{array}$ & $\begin{array}{l}37,46 \\
46,21\end{array}$ & 297, \\
\hline $\begin{array}{l}\text { Turm von Hanoi }(\mathrm{TvH}) \\
\text { vorher } \\
\text { Turm von Hanoi }(\mathrm{TvH}) \\
\text { nachher }\end{array}$ & 8 & $\begin{array}{r}12,44 \\
8,00\end{array}$ & $\begin{array}{l}6,20 \\
3,00\end{array}$ & ,067 \\
\hline
\end{tabular}

$\mathrm{N}=$ Anzahl der Probanden; MW=Mittelwert; SD=Standardabweichung 
Die exekutiven Funktionen der PD-Patienten in der Kontrollbedingung sind nach 4 Monaten größtenteils signifikant schlechter geworden. Auffällig ist eine reduzierte Kapazität des verbalen Arbeitsgedächtnisses (MW vorher $=5,87$, $\mathrm{SD}=1,24 ; \quad \mathrm{MW}$ nachher $=4,75, \quad \mathrm{SD}=1,28 ; \mathrm{p}<0,05)$ und des visuellen Arbeitsgedächtnisses ( $M W$ vorher $=8,00, S D=1,85 ; \mathrm{MW}$ nachher $=6,12$, $S D=2,10 ;$ p $<.01$ ), (Abb. 17 u. 18; Tab. 38).

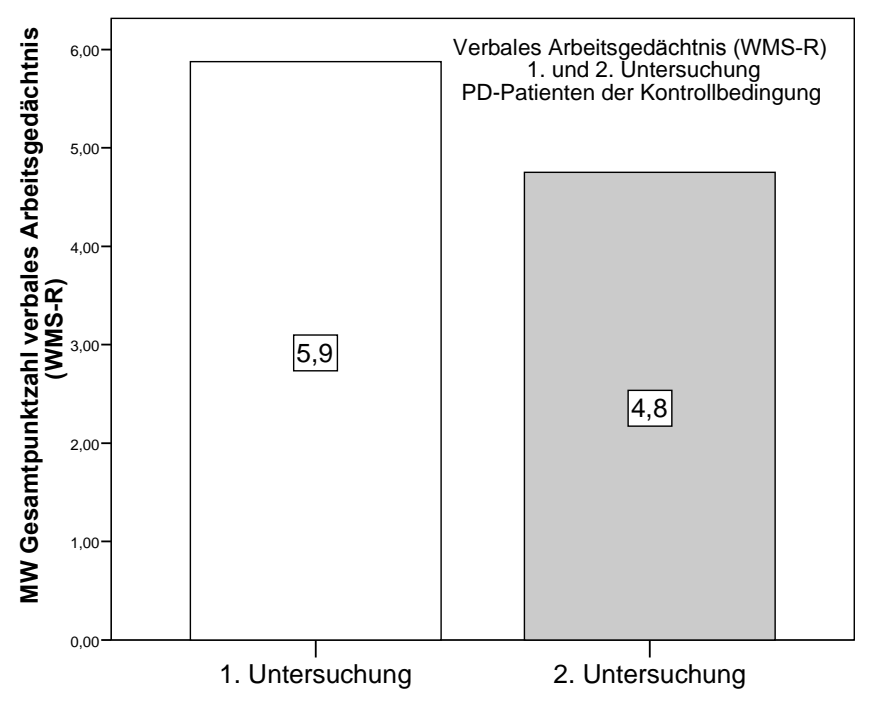

Abb.17 Verbales Arbeitsgedächtnis der PD-Patienten in der Kontrollgruppe in der 1 . und 2. Untersuchung $(p=0,026)$ 


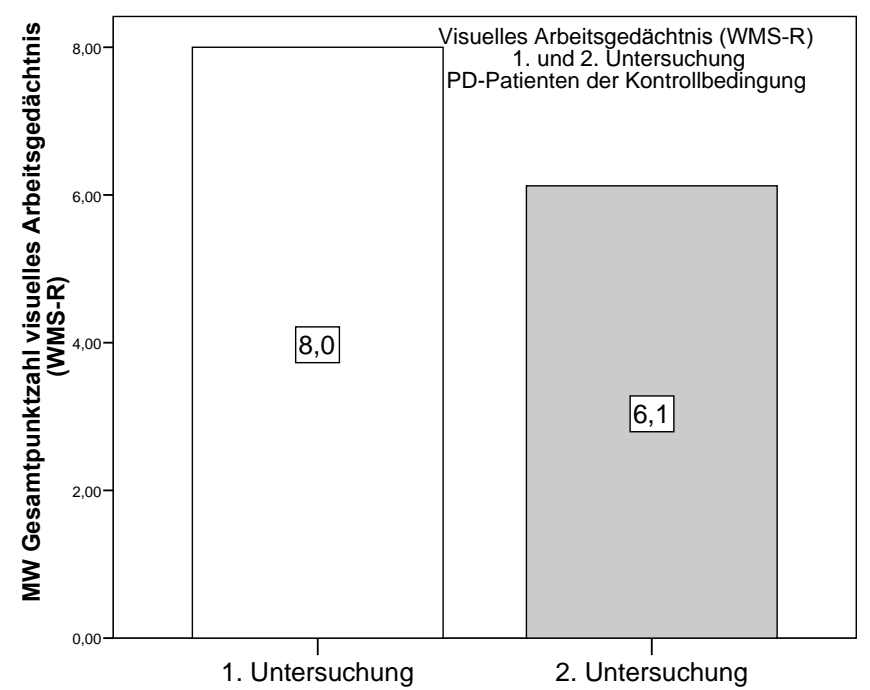

Abb.18 Visuelles Arbeitsgedächtnis der PD-Patienten in der Kontrollgruppe in der 1. und 2. Untersuchung $(p=0,008)$

Darüber hinaus verschlechterte sich die kognitive Flexibilität (TMT-B) in der Gruppe der PD-Patienten in Kontrollbedingung signifikant (MW vorher $=168,00$ sec, $S D=93,85 \mathrm{sec} ; \mathrm{MW}$ nachher = 181,00sec, $S D=19,91 \mathrm{sec} ; \mathrm{p}<.01) ;($ Abb. 19; Tab. 38).

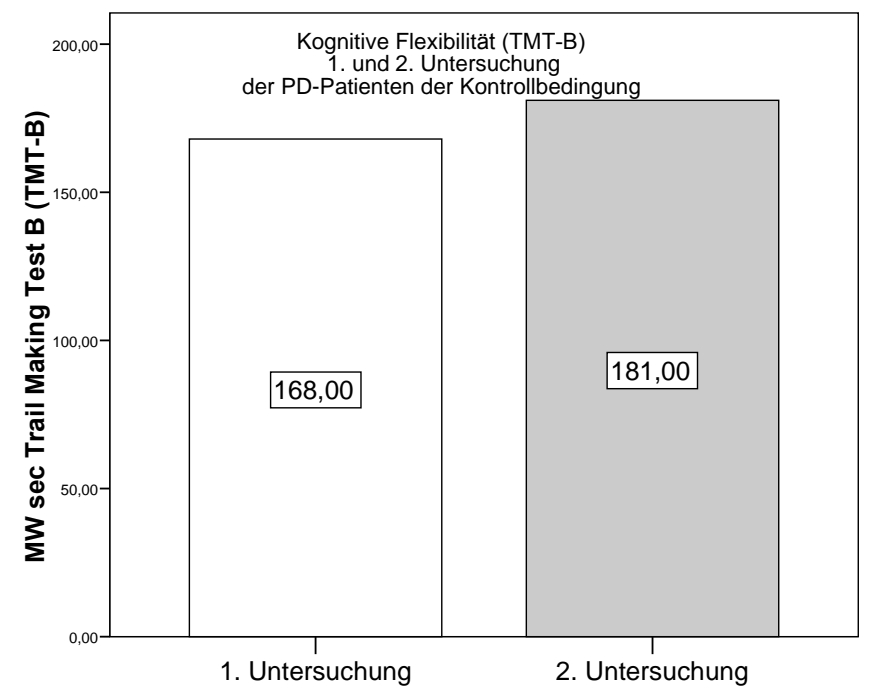

Abb.19 Kognitive Flexibilität der PD-Patienten in der Kontrollgruppe in der 1. und 2. Untersuchung $(p=0,008)$ 
In der Aufgabe zum planerischen Handeln $(\mathrm{TvH})$ haben sich die Leistungen in dieser Gruppe nicht reduziert (MW vorher $=12,00, \mathrm{SD}=5,78 ; \mathrm{MW}$ nachher $=8,25$, $\mathrm{SD}=3,15, \mathrm{p}=.187) ;($ Tab. 38).

Tab.38 Mittelwerte der exekutiven Funktionen der PD-Patienten in Kontrollbedingung, gemessen mit WMS-R, TMT-B, TvH in der 1. und 2. neuropsychologischen Untersuchung

\begin{tabular}{|c|c|c|c|c|}
\hline $\begin{array}{l}\text { 1. und 2. neuropsychologische Untersuchung } \\
\text { Testverfahren }\end{array}$ & $\begin{array}{l}\text { PD-Patienten } \\
\text { Kontrollgruppe } \\
\mathrm{N}\end{array}$ & MW & SD & $\begin{array}{l}\text { Signifikanz } \\
\text { 2-seitig }\end{array}$ \\
\hline $\begin{array}{l}\text { Verbales Arbeitsgedächtnis (WMS-R) } \\
\text { vorher } \\
\text { Verbales Arbeitsgedächtnis (WMS-R) } \\
\text { Nachher }\end{array}$ & 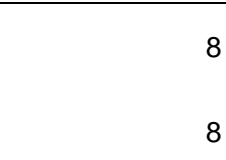 & 5,87 & 1,24 & ,026, \\
\hline $\begin{array}{l}\text { Visuelles Arbeitsgedächtnis (WMS-R) } \\
\text { vorher } \\
\text { Visuelles Arbeitsgedächtnis (WMS-R) } \\
\text { nachher }\end{array}$ & 8 & 8,00 & 1,85 & ,008, \\
\hline $\begin{array}{l}\text { Trail Making Test B (TMT-B) } \\
\text { Vorher } \\
\text { Trail Making Test B (TMT-B) } \\
\text { Nachher }\end{array}$ & 8 & $\begin{array}{l}168,00 \\
181,00\end{array}$ & $\begin{array}{r}93,85 \\
19,91\end{array}$ & ,008, \\
\hline $\begin{array}{l}\text { Turm von Hanoi }(\mathrm{TvH}) \\
\text { Vorher } \\
\text { Turm von Hanoi }(\mathrm{TvH}) \\
\text { Nachher }\end{array}$ & 8 & $\begin{array}{r}12,00 \\
8,25\end{array}$ & $\begin{array}{l}5,78 \\
3,15\end{array}$ & 187, \\
\hline
\end{tabular}

$\mathrm{N}=$ Anzahl der Probanden; MW=Mittelwert; SD=Standardabweichung

Die exekutiven Funktionen der gesunden VPN in Therapiebedingung haben sich nach 4 Monaten größtenteils signifikant verbessert, z.B. die Kapazität des verbalen Arbeitsgedächtnisses ( $\mathrm{MW}$ vorher $=6,14, \mathrm{SD}=1,46$; $\mathrm{MW}$ nachher = $8,00, S D=2,15 ; p<0,05)$ und des visuellen Arbeitsgedächtnisses (MW vorher $=$ 6,28, SD=1,11; MW nachher = 7,14, SD=0,89; p<.05); (Abb. 20 u. 21; Tab. 39). 


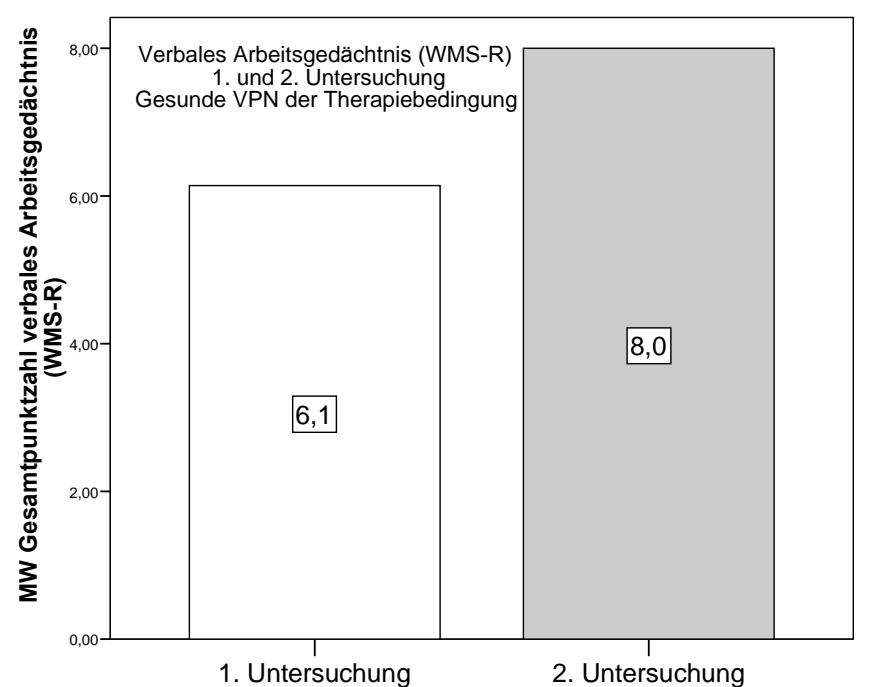

Abb.20 Verbales Arbeitsgedächtnis der gesunden VPN der Therapiegruppen in der 1 . und 2 . Untersuchung $(p=0,032)$

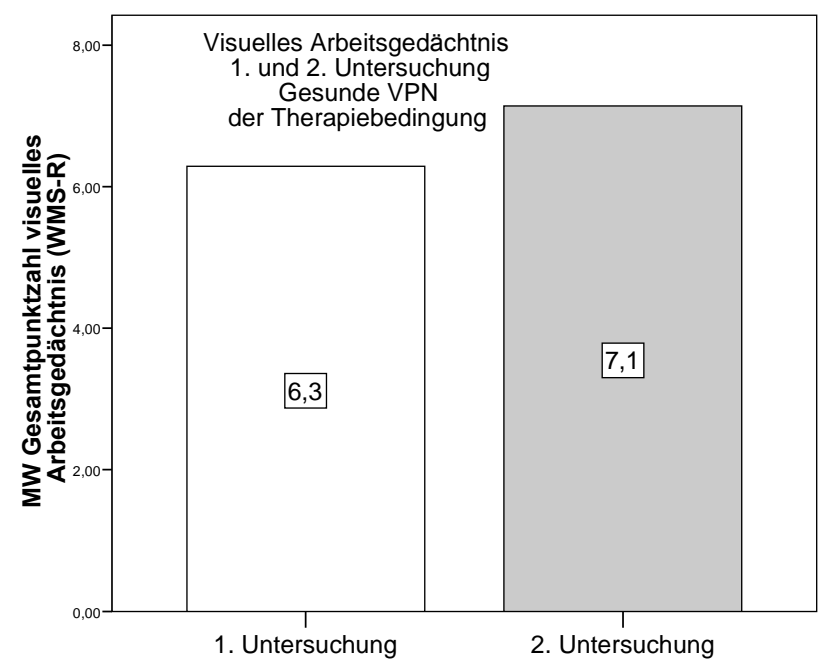

Abb.21 Visuelles Arbeitsgedächtnis der gesunden VPN der Therapiegruppen in der 1. u. 2. Untersuchung $(p=0,045)$

Darüber hinaus verbesserte sich die kognitive Flexibilität signifikant (MW vorher $=117,71 \mathrm{sec}, \mathrm{SD}=28,46 \mathrm{sec} ; \mathrm{MW}$ nachher $=83,71 \mathrm{sec}, \mathrm{SD}=21,92 \mathrm{sec} ; \mathrm{p}<.05)$; (Abb. 22; Tab. 39). 


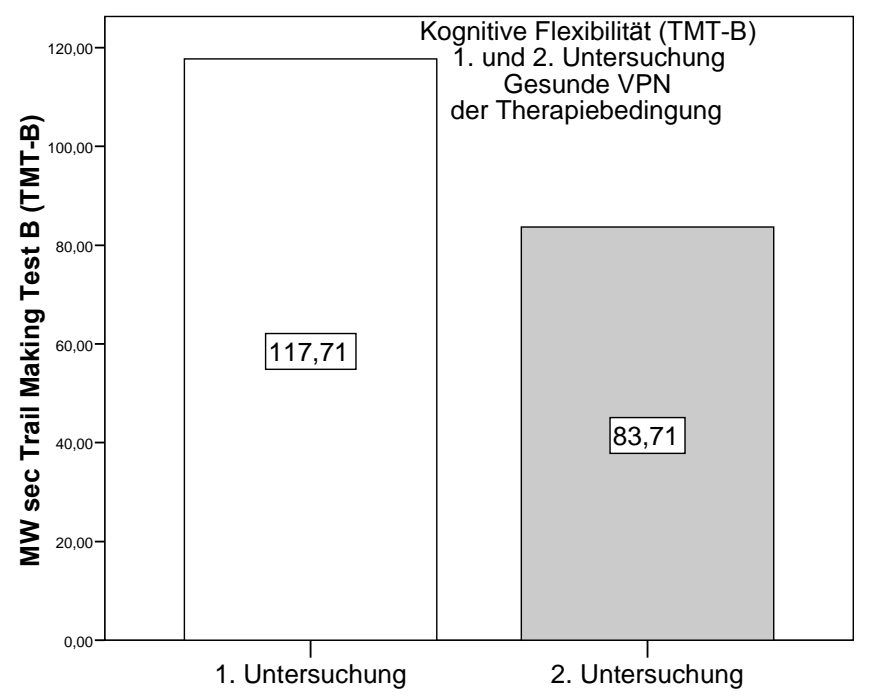

Abb.22 Kognitive Flexibilität der gesunden VPN der Therapiegruppen in der 1. und 2. Untersuchung $(p=0,014)$

Tab.39 Mittelwerte der exekutiven Funktionen der gesunden VPN in den Therapiegruppen, gemessen mit WMS$\mathrm{R}, \mathrm{TMT}-\mathrm{B}, \mathrm{TVH}$ in der 1. und 2. neuropsychologischen Untersuchung

\begin{tabular}{|c|c|c|c|c|}
\hline $\begin{array}{l}\text { 1. und 2. neuropsychologische Untersuchung } \\
\text { Testverfahren }\end{array}$ & $\begin{array}{l}\text { Gesunde VPN } \\
\text { Therapiegruppen } \\
\mathrm{N}\end{array}$ & MW & SD & $\begin{array}{l}\text { Signifikanz } \\
\text { 2-seitig }\end{array}$ \\
\hline $\begin{array}{l}\text { Verbales Arbeitsgedächtnis (WMS-R) } \\
\text { Vorher } \\
\text { Verbales Arbeitsgedächtnis (WMS-R) } \\
\text { Nachher }\end{array}$ & 8 & $\begin{array}{l}6,14 \\
8,00\end{array}$ & $\begin{array}{l}1,46 \\
2,16\end{array}$ & ,032 \\
\hline $\begin{array}{l}\text { Visuelles Arbeitsgedächtnis (WMS-R) } \\
\text { Vorher } \\
\text { Visuelles Arbeitsgedächtnis (WMS-R) } \\
\text { Nachher }\end{array}$ & 8 & $\begin{array}{l}6,28 \\
7,14\end{array}$ & $\begin{array}{r}1,11 \\
, 89\end{array}$ & ,045 \\
\hline $\begin{array}{l}\text { Trail-Making-Test B (TMT-B ) } \\
\text { Vorher } \\
\text { Trail-Making-Test B (TMT-B) } \\
\text { Nachher }\end{array}$ & 8 & $\begin{array}{r}117,71 \\
83,71\end{array}$ & $\begin{array}{l}28,46 \\
21,92\end{array}$ & ,014 \\
\hline $\begin{array}{l}\text { Turm von Hanoi }(\mathrm{TvH}) \\
\text { Vorher } \\
\text { Turm von Hanoi }(\mathrm{TvH}) \\
\text { Nachher }\end{array}$ & 8 & 7,57 &, 97 & 172, \\
\hline
\end{tabular}

$\mathrm{N}=$ Anzahl der Probanden; MW=Mittelwert; $\mathrm{SD}=$ Standardabweichung 
Tab. 39 zeigt ferner, dass in der Aufgabe zum planerischen Handeln (TvH) keine weitere signifikante Verbesserung beobachtet werden konnte (MW vorher $=7,00$, $\mathrm{SD}=0 ; \mathrm{MW}$ nachher $=7,57, \mathrm{SD}=0,97 ; \mathrm{p}=.172)$.

Die Leistungen in den exekutiven Funktionen der gesunden VPN in der Kontrollgruppe haben sich nach 4 Monaten insgesamt nicht signifikant verändert; . (Tab. 40).

Tab.40 Mittelwerte der exekutiven Funktionen der gesunden VPN in der Kontrollbedingung, gemessen mit WMS$\mathrm{R}, \mathrm{TMT}-\mathrm{B}, \mathrm{TVH}$ in der 1. und 2. neuropsychologischen Untersuchung

\begin{tabular}{|c|c|c|c|c|}
\hline $\begin{array}{l}\text { 1. und 2. neuropsychologische Untersuchung } \\
\text { Testverfahren }\end{array}$ & $\begin{array}{l}\text { Gesunde VPN } \\
\text { Kontrollgruppe } \\
\mathrm{N}\end{array}$ & MW & SD & $\begin{array}{c}\text { Signifikanz } \\
\text { 2-seitig }\end{array}$ \\
\hline $\begin{array}{l}\text { Verbales Arbeitsgedächtnis (WMS-R) } \\
\text { Vorher }\end{array}$ & 8 & 6,50 & ,92 & 142, \\
\hline $\begin{array}{l}\text { Verbales Arbeitsgedächtnis (WMS-R) } \\
\text { Nachher }\end{array}$ & 8 & 5,75 & ,70 & \\
\hline $\begin{array}{l}\text { Visuelles Arbeitsgedächtnis (WMS-R) } \\
\text { Vorher }\end{array}$ & 8 & 6,37 & 1,92 & ,741 \\
\hline $\begin{array}{l}\text { Visuelles Arbeitsgedächtnis (WMS-R) } \\
\text { Nachher }\end{array}$ & 8 & 6,62 & ,91 & \\
\hline $\begin{array}{l}\text { Trail-Making-Test B (TMT-B) } \\
\text { Vorher }\end{array}$ & 8 & 98,87 & 32,28 & ,333 \\
\hline $\begin{array}{l}\text { Trail-Making-Test B (TMT-B) } \\
\text { Nachher }\end{array}$ & 8 & 107,75 & 45,72 & \\
\hline $\begin{array}{l}\text { Turm von Hanoi (TvH) } \\
\text { Vorher }\end{array}$ & 8 & 9,12 & 3,68 & 901 \\
\hline $\begin{array}{l}\text { Turm von Hanoi (TvH) } \\
\text { Nachher }\end{array}$ & 8 & 8,87 & 2,74 & \\
\hline
\end{tabular}

$\mathrm{N}=$ Anzahl der Probanden; MW=Mittelwert; SD=Standardabweichung

\subsection{Psychische Aspekte}

\subsubsection{Psychische Aspekte in der 1. Untersuchung}

In der 1. neuropsychologischen Untersuchung unterschieden sich die PDPatienten der Therapie- und Kontrollbedingung im Hinblick auf die Depressionsneigung, gemessen dem Beck'schen Depressions-Inventar (BDI) 
nicht signifikant voneinander. Auch zeigte sich bzgl. der State-Angst, gemessen dem Stait-Trait-Angstinventar (STAI) kein Unterschied. Die Testauswertung zur partnerschaftlichen Zufriedenheit, gemessen dem Fragebogen zur Lebenszufriedenheit (FLZ-Subskala „Ehe und Partnerschaft“), ergab ebenfalls keinen signifikanten Unterschied; (Tab. 41).

Tab.41 Mittelwerte der mittels Fragebögen ermittelten psychischen Aspekte der PD-Patienten der Kontroll- und Therapiebedingung, gemessen mit BDI, STAI und FLZ in der 1. neuropsychologischen Untersuchung

\begin{tabular}{|l|l|r|r|r|r|}
\hline $\begin{array}{c}\text { 1. neuropsychologische } \\
\text { Untersuchung } \\
\text { Testverfahren }\end{array}$ & \multicolumn{1}{|c|}{ PD-Patienten } & N & MW & SD & $\begin{array}{c}\text { Signifikanz } \\
\text { 2-seitig }\end{array}$ \\
\hline $\begin{array}{l}\text { Depressionsfragebogen } \\
\text { (BDI) } \\
\text { vorher }\end{array}$ & Kontrollgruppe & 8 & 11,00 & 6,80 &, 724 \\
\hline $\begin{array}{l}\text { State Angst } \\
\text { (STAI) }\end{array}$ & Therapiegruppen & 8 & 12,44 & 9,34 &, 335 \\
vorher & Kontrollgruppe & 8 & 41,87 & 10,50 & 10,64 \\
\hline $\begin{array}{l}\text { Lebenszufriedenheit } \\
\text { Ehe und Partnerschaft } \\
\text { (FLZ) vorher }\end{array}$ & Therapiegruppen & 8 & 47,00 & 4,95 &, 079 \\
\hline
\end{tabular}

$\mathrm{N}=$ Anzahl der Probanden; MW=Mittelwert; SD=Standardabweichung

Die gesunden VPN der Therapie- und Kontrollbedingung zeigten in der Depressionsneigung, in der Ängstlichkeit und in Zufriedenheit in der Partnerschaft eine ähnliche Ausprägung; (Tab. 42).

Tab.42 Mittelwerte der mittels Fragebögen ermittelten psychischen Aspekte der gesunden VPN der Kontroll- und Therapiebedingung, gemessen mit BDI, STAI und FLZ in der 1.neuropsychologischen Untersuchung

\begin{tabular}{|c|c|c|c|c|c|}
\hline $\begin{array}{l}\text { 1. neuropsychologische } \\
\text { Untersuchung } \\
\text { Testverfahren }\end{array}$ & Gesunde VPN & $\mathrm{N}$ & MW & SD & $\begin{array}{l}\text { Signifikanz } \\
\text { 2-seitig }\end{array}$ \\
\hline \multirow{2}{*}{$\begin{array}{l}\text { Depressionsfragebogen } \\
\text { (BDI) }\end{array}$} & Kontrollgruppe & 8 & 9,25 & 6,22 & \multirow{2}{*}{,820 } \\
\hline & Therapiegruppen & 8 & 10,00 & 6,21 & \\
\hline \multirow{2}{*}{$\begin{array}{l}\text { State Angst } \\
\text { (STAI) }\end{array}$} & Kontrollgruppe & 8 & 36,75 & 8,06 & \multirow{2}{*}{,056 } \\
\hline & Therapiegruppen & 8 & 45,42 & 7,93 & \\
\hline \multirow{2}{*}{$\begin{array}{l}\text { Lebenszufriedenheit } \\
\text { Ehe und Partnerschaft } \\
\text { (FLZ) }\end{array}$} & Kontrollgruppe & 8 & 40,75 & 4,26 & \multirow{2}{*}{,395 } \\
\hline & Therapiegruppen & 8 & 38,14 & 7,05 & \\
\hline
\end{tabular}

$\mathrm{N}=$ Anzahl der Probanden; MW=Mittelwert; SD=Standardabweichung 
Die PD-Patienten der Therapiegruppen zeigten in ihrer Depressionsneigung, der Ängstlichkeit sowie der Zufriedenheit in der Partnerschaft im Vergleich zu den gesunden VPN der Therapiegruppen keine signifikanten Unterschiede; (Tab. 43).

Tab.43 Mittelwerte der mittels Fragebögen ermittelten psychischen Aspekte der PD-Patienten und der gesunden VPN der Therapiebedingung, gemessen mit BDI, STAI und FLZ in der 1. neuropsychologischen Untersuchung

\begin{tabular}{|c|c|c|c|c|c|}
\hline $\begin{array}{l}\text { 1. neuropsychologische } \\
\text { Untersuchung } \\
\text { Testverfahren }\end{array}$ & $\begin{array}{c}\text { VPN } \\
\text { Therapiegruppen }\end{array}$ & $\mathrm{N}$ & MW & SD & $\begin{array}{l}\text { Signifikanz } \\
\text { 2-seitig }\end{array}$ \\
\hline \multirow{2}{*}{$\begin{array}{l}\text { Depressionsfragebogen } \\
\text { (BDI) }\end{array}$} & Gesunde VPN & 8 & 10,00 & 6,21 & \multirow{2}{*}{, 561} \\
\hline & PD-Patienten & 8 & 12,44 & 9,34 & \\
\hline \multirow{2}{*}{$\begin{array}{l}\text { State Angst } \\
\text { (STAI) }\end{array}$} & Gesunde VPN & 8 & 45,42 & 7,93 & \multirow{2}{*}{, 750} \\
\hline & PD-Patienten & 8 & 47,00 & 10,64 & \\
\hline \multirow{2}{*}{$\begin{array}{l}\text { Lebenszufriedenheit Ehe } \\
\text { und Partnerschaft } \\
\text { (FLZ) }\end{array}$} & Gesunde VPN & 8 & 38,14 & 7,05 & \multirow{2}{*}{,909 } \\
\hline & PD-Patienten & 8 & 37,77 & 5,56 & \\
\hline
\end{tabular}

$\mathrm{N}=$ Anzahl der Probanden; MW=Mittelwert; SD=Standardabweichung

Die gesunden VPN der Kontrollbedingung unterschieden sich im Hinblick auf die Depressionsneigung, die Ängstlichkeit und die Lebenszufriedenheit in der Partnerschaft von den PD-Patienten der Kontrollbedingung nicht voneinander; (Tab. 44).

Tab.44 Mittelwerte der mittels Fragebögen ermittelten psychischen Aspekte der PD-Patienten und der gesunden VPN der Kontrollbedingung, gemessen mit BDI, STAI und FLZ in der 1. neuropsychologischen Untersuchung

\begin{tabular}{|l|l|r|r|r|r|}
\hline $\begin{array}{c}\text { 1. neuropsychologische } \\
\text { Untersuchung } \\
\text { Testverahren }\end{array}$ & \multicolumn{1}{c|}{$\begin{array}{c}\text { VPN } \\
\text { Kontrollgruppe }\end{array}$} & N & MW & SD & $\begin{array}{c}\text { Signifikanz } \\
\text { 2-seitig }\end{array}$ \\
\hline $\begin{array}{l}\text { Depressionsfragebogen } \\
\text { (BDI) }\end{array}$ & Gesunde VPN & 8 & 9,25 & 6,22 &, 600 \\
& PD-Patienten & 8 & 11,00 & 6,80 &, 292 \\
\hline State Angst & Gesunde VPN & 8 & 36,75 & 8,06 &, 431 \\
(STAl) & PD-Patienten & 8 & 41,87 & 10,50 & 4,26 \\
\hline Lebenszufriedenheit Ehe & Gesunde VPN & 8 & 40,75 & 4,95 & \\
und Partnerschaft (FLZ) & PD-Patienten & 8 & 42,62 & & \\
\hline
\end{tabular}

$\mathrm{N}=$ Anzahl der Probanden; MW=Mittelwert; SD=Standardabweichung 


\subsubsection{Psychische Aspekte in der 2. Untersuchung}

Nach Beendigung der Therapie konnten die PD-Patienten der Therapiegruppen im Vergleich zu den PD-Patienten der Kontrollgruppe in der Depressionsneigung keine signifikante Verbesserung erzielen; sie erreichten jedoch einen tendenziell niedrigeren Mittelwert. Hinsichtlich der Ängstlichkeit unterschieden sich die PDPatienten der Therapiegruppen bzgl. ihrer State-Angst und der partnerschaftlichen Zufriedenheit ebenfalls nicht signifikant von den PD-Patienten der Kontrollgruppe; (Tab. 45).

Tab.45 Mittelwerte der mittels Fragebögen ermittelten psychischen Aspekte der PD-Patienten in der Kontrollund Therapiebedingung, gemessen mit BDI, STAI und FLZ in der 2. neuropsychologischen Untersuchung

\begin{tabular}{|c|c|c|c|c|c|}
\hline $\begin{array}{l}\text { 2. neuropsychologische } \\
\text { Untersuchung } \\
\text { Testverfahren }\end{array}$ & PD-Patienten & $\mathrm{N}$ & MW & SD & $\begin{array}{l}\text { Signifikanz } \\
\text { 2-seitig }\end{array}$ \\
\hline \multirow{2}{*}{$\begin{array}{l}\text { Depressionsfragebogen } \\
\text { (BDI) }\end{array}$} & Kontrollgruppe & 8 & 11,87 & 5,84 & \multirow{2}{*}{, 571} \\
\hline & Therapiegruppen & 8 & 9,55 & 9,86 & \\
\hline \multirow{2}{*}{$\begin{array}{l}\text { State Angst } \\
\text { (STAI) }\end{array}$} & Kontrollgruppe & 8 & 43,25 & 14,22 & \multirow{2}{*}{,783 } \\
\hline & Therapiegruppen & 8 & 45,00 & 11,53 & \\
\hline \multirow{2}{*}{$\begin{array}{l}\text { Lebenszufriedenheit } \\
\text { Ehe und Partnerschaft } \\
\text { (FLZ) }\end{array}$} & Kontrollgruppe & 8 & 42,12 & 5,64 & \multirow{2}{*}{, 141 } \\
\hline & Therapiegruppen & 8 & 38,22 & 4,71 & \\
\hline
\end{tabular}

$\mathrm{N}=$ Anzahl der Probanden; MW=Mittelwert; SD=Standardabweichung

Die gesunden VPN der Kontrollbedingung unterschieden sich im Hinblick auf die Depressionsneigung, Ängstlichkeit sowie Zufriedenheit in der Partnerschaft nicht signifikant von den gesunden VPN der Therapiebedingung; (Tab. 46).

Tab.46 Mittelwerte der mittels Fragebögen ermittelten psychischen Aspekte der gesunden VPN in der Kontrollund Therapiebedingung, gemessen mit BDI, STAI und FLZ in der 2. neuropsychologischen Untersuchung

\begin{tabular}{|c|c|c|c|c|c|}
\hline $\begin{array}{l}\text { 2. neuropsychologische } \\
\text { Untersuchung } \\
\text { Testverfahren }\end{array}$ & Gesunde VPN & $\mathrm{N}$ & MW & SD & $\begin{array}{l}\text { Signifikanz } \\
\text { 2-seitig }\end{array}$ \\
\hline \multirow{2}{*}{$\begin{array}{l}\text { Depressionsfragebogen } \\
\text { (BDI) }\end{array}$} & Kontrollgruppe & 8 & 8,00 & 6,23 & \multirow{2}{*}{,921 } \\
\hline & Therapiegruppen & 8 & 8,28 & 4,46 & \\
\hline \multirow{2}{*}{$\begin{array}{l}\text { State Angst } \\
\text { (STAI) }\end{array}$} & Kontrollgruppe & 8 & 35,12 & 10,26 & \multirow{2}{*}{,564 } \\
\hline & Therapiegruppen & 8 & 38,57 & 12,32 & \\
\hline \multirow{2}{*}{$\begin{array}{l}\text { Lebenszufriedenheit } \\
\text { Ehe und Partnerschaft } \\
\text { (FLZ) }\end{array}$} & Kontrollgruppe & 8 & 42,62 & 4,10 & \multirow{2}{*}{,085 } \\
\hline & Therapiegruppen & 8 & 37,85 & 5,75 & \\
\hline
\end{tabular}

$\mathrm{N}=$ Anzahl der Probanden; $\mathrm{MW}=$ Mittelwert; SD=Standardabweichung 
In der 2. Untersuchung der Therapiegruppen unterschieden sich die PDPatienten im Hinblick auf Depressionsneigung, State-Angst und der partnerschaftlichen Zufriedenheit nicht signifikant von den gesunden VPN, wiesen jedoch bei der Depressionneigung und der Ängstlichkeit tendenziell höhere Werte auf. Die Zufriedenheit in der Partnerschaft war bei den Patienten tendenziell besser ausgeprägt; (Tab. 47).

Tab.47 Mittelwerte der mittels Fragebögen ermittelten psychischen Aspekte der PD-Patienten und der gesunden VPN in der Therapiebedingung, gemessen mit BDI, STAI und FLZ in der 2. neuropsychologischen Untersuchung

\begin{tabular}{|c|c|c|c|c|c|}
\hline $\begin{array}{l}\text { 2. neuropsychologische } \\
\text { Untersuchung } \\
\text { Testverfahren }\end{array}$ & $\begin{array}{c}\text { VPN } \\
\text { Therapiegruppen }\end{array}$ & $\mathrm{N}$ & MW & SD & $\begin{array}{l}\text { Signifikanz } \\
\text { 2-seitig }\end{array}$ \\
\hline \multirow{2}{*}{$\begin{array}{l}\text { Depressionsfragebogen } \\
\text { (BDI) }\end{array}$} & Gesunde VPN & 8 & 8,28 & 4,46 & \multirow{2}{*}{,758 } \\
\hline & PD-Patienten & 8 & 9,55 & 9,86 & \\
\hline \multirow{2}{*}{$\begin{array}{l}\text { State Angst } \\
\text { (STAI) }\end{array}$} & Gesunde VPN & 8 & 38,57 & 12,32 & \multirow[b]{2}{*}{,301 } \\
\hline & PD-Patienten & 8 & 45,00 & 11,53 & \\
\hline \multirow{2}{*}{$\begin{array}{l}\text { Lebenszufriedenheit Ehe } \\
\text { und Partnerschaft (FLZ) }\end{array}$} & Gesunde VPN & 8 & 37,85 & 5,75 & \multirow{2}{*}{,891 } \\
\hline & PD-Patienten & 8 & 38,22 & 4,71 & \\
\hline
\end{tabular}

$\mathrm{N}=$ Anzahl der Probanden; MW=Mittelwert; SD=Standardabweichung

Auch die PD-Patienten der Kontrollbedingung unterschieden sich im Hinblick auf die Depressionsneigung, Ängstlichkeit und die Zufriedenheit in der Partnerschaft nicht signifikant von den gesunden VPN der Kontrollbedingung, wobei sie jedoch insgesamt tendenziell schlechtere Werte erreichten; (Tab. 48).

Tab.48 Mittelwerte der mittels Fragebögen ermittelten psychischen Aspekte der PD-Patienten und der gesunden VPN in der Kontrollbedingung, gemessen mit BDI, STAI und FLZ in der 2. neuropsychologischen Untersuchung

\begin{tabular}{|c|c|c|c|c|c|}
\hline $\begin{array}{l}\text { 2. neuropsychologische } \\
\text { Untersuchung } \\
\text { Testverfahren }\end{array}$ & $\begin{array}{c}\text { VPN } \\
\text { Kontrollgruppe }\end{array}$ & $\mathrm{N}$ & MW & SD & $\begin{array}{l}\text { Signifikanz } \\
\text { 2-seitig }\end{array}$ \\
\hline \multirow{2}{*}{$\begin{array}{l}\text { Depressionsfragebogen } \\
\text { (BDI) }\end{array}$} & \multirow{2}{*}{$\begin{array}{l}\text { Gesunde VPN } \\
\text { PD-Patienten }\end{array}$} & 8 & 8,00 & 6,23 & \multirow{2}{*}{,220 } \\
\hline & & 8 & 11,87 & 5,84 & \\
\hline \multirow{2}{*}{$\begin{array}{l}\text { State Angst } \\
\text { (STAI) }\end{array}$} & \multirow{2}{*}{$\begin{array}{l}\text { Gesunde VPN } \\
\text { PD-Patienten }\end{array}$} & 8 & 35,12 & 10,26 & \multirow{2}{*}{,211 } \\
\hline & & 8 & 43,25 & 14,22 & \\
\hline \multirow{2}{*}{$\begin{array}{l}\text { Lebenszufriedenheit Ehe } \\
\text { und Partnerschaft (FLZ) }\end{array}$} & Gesunde VPN & 8 & 42,62 & 4,10 & \multirow{2}{*}{,842 } \\
\hline & PD-Patienten & 8 & 42,12 & 5,64 & \\
\hline
\end{tabular}

$\mathrm{N}=$ Anzahl der Probanden; $\mathrm{MW}=$ Mittelwert; $\mathrm{SD}=$ Standardabweichung 


\subsubsection{Vergleich der psychischen Aspekte in der 1. und 2. Untersuchung}

Der Vergleich zwischen der Befindlichkeit vor Therapiebeginn und nach Therapieende ergab hinsichtlich Depression, Ängstlichkeit und Zufriedenheit in der Partnerschaft für die Patienten der Therapiebedingung tendenziell verbesserte Werte in allen Bereichen; (Tab. 49).

Tab.49 Mittelwerte der PD-Patienten in Therapiebedingung, gemessen mit BDI, STAI, FLZ in der 1. und 2. neuropsychologischen Untersuchung

\begin{tabular}{|c|c|c|c|c|}
\hline $\begin{array}{l}\text { 1. und 2. neuropsychologische Untersuchung } \\
\text { Testverfahren }\end{array}$ & $\begin{array}{l}\text { PD-Patienten } \\
\text { Therapiegruppen } \\
\mathrm{N}\end{array}$ & MW & SD & $\begin{array}{l}\text { Signifikanz } \\
\text { 2-seitig }\end{array}$ \\
\hline $\begin{array}{l}\text { Depressionsfragebogen } \\
\text { (BDI) vorher }\end{array}$ & 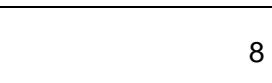 & 12,44 & 9,34 & \\
\hline $\begin{array}{l}\text { Depressionsfragebogen } \\
\text { (BDI) nachher }\end{array}$ & 8 & 9,55 & 9,86 & 088, \\
\hline $\begin{array}{l}\text { State Angst } \\
\text { (STAI) vorher }\end{array}$ & 8 & 47,00 & 10,64 & \\
\hline $\begin{array}{l}\text { State Angst } \\
\text { (STAI) nachher }\end{array}$ & 8 & 45,00 & 11,53 & ,524 \\
\hline $\begin{array}{l}\text { Lebenszufriedenheit Ehe und Partnerschaft } \\
\text { (FLZ) vorher }\end{array}$ & 8 & 37,77 & 5,56 & \\
\hline $\begin{array}{l}\text { Lebenszufriedenheit Ehe und Partnerschaft } \\
\text { (FLZ) nachher }\end{array}$ & 8 & 38,22 & 4,71 & ,711 \\
\hline
\end{tabular}

$\mathrm{N}=$ Anzahl der Probanden; MW=Mittelwert; SD=Standardabweichung

Im Vergleich zu der an der Therapie teilnehmenden Gruppe der PD-Patienten ergab sich in der PD-Kontrollgruppe bei der Auswertung der Fragebögen zur psychischen Befindlichkeit tendenzielle Verschlechterungen hinsichtlich der psychischen Aspekte. Die Zufriedenheit in der Partnerschaft blieb jedoch annähernd gleich; (Tab. 50). 
Tab.50 Mittelwerte der PD-Patienten in Kontrollbedingung, gemessen mit BDI, STAI, FLZ, in der 1. und 2. neuropsychologischen Untersuchung

\begin{tabular}{|l|r|r|r|r|}
\hline $\begin{array}{l}\text { 1. und 2. neuropsychologische Untersuchung } \\
\text { Testverfahren }\end{array}$ & $\begin{array}{c}\text { PD-Patienten } \\
\text { Kontrollgruppe }\end{array}$ & MW & SD & $\begin{array}{c}\text { Signifikanz } \\
\text { 2-seitig }\end{array}$ \\
\hline $\begin{array}{l}\text { Depressionsfragebogen } \\
\text { (BDI) vorher }\end{array}$ & 8 & 11,00 & 6,80 &, 490 \\
$\begin{array}{l}\text { Depressionsfragebogen } \\
\text { (BDI) nachher }\end{array}$ & 8 & 11,87 & 5,84 & \\
\hline $\begin{array}{l}\text { State Angst } \\
\text { (STAl) vorher }\end{array}$ & 8 & 41,87 & 10,50 &, 805 \\
$\begin{array}{l}\text { State Angst } \\
\text { (STAl) nachher }\end{array}$ & 8 & 43,25 & 14,22 & \\
\hline $\begin{array}{l}\text { Lebenszufriedenheit Ehe und Partnerschaft } \\
\text { (FLZ) vorher }\end{array}$ & 8 & 42,62 & 4,95 &, 708 \\
$\begin{array}{l}\text { Lebenszufriedenheit Ehe und Partnerschaft } \\
\text { (FLZ) nachher }\end{array}$ & 8 & 42,12 & 5,64 & \\
\hline
\end{tabular}

$\mathrm{N}=$ Anzahl der Probanden; MW=Mittelwert; SD=Standardabweichung

Die gesunden VPN der Therapiebedingung zeigten in der 2. Untersuchung im Vergleich zur ersten Untersuchung in der Depressionsneigung und Ängstlichkeit keine signifikanten Unterschiede, jedoch tendenziell bessere Werte. Die Zufriedenheit in der Partnerschaft stellte sich als geringgradig weniger gut dar; (Tab. 51).

Tab.51 Mittelwerte der gesunden VPN in Therapiebedingung, gemessen mit BDI, STAI, FLZ in der 1. und 2. neuropsychologischen Untersuchung

\begin{tabular}{|c|c|c|c|c|}
\hline $\begin{array}{l}\text { 1. und 2. neuropsychologische Untersuchung } \\
\text { Testverfahren }\end{array}$ & $\begin{array}{l}\text { Gesunde VPN } \\
\text { Therapiegruppen } \\
\text { N }\end{array}$ & MW & SD & $\begin{array}{l}\text { Signifikanz } \\
\text { 2-seitig }\end{array}$ \\
\hline $\begin{array}{l}\text { Depressionsfragebogen } \\
\text { (BDI) vorher } \\
\text { Depressionsfragebogen } \\
\text { (BDI) nachher }\end{array}$ & 8 & $\begin{array}{r}10,00 \\
8,28\end{array}$ & $\begin{array}{l}6,21 \\
4,46\end{array}$ & 349, \\
\hline $\begin{array}{l}\text { State Angst } \\
\text { (STAI) vorher } \\
\text { State Angst } \\
\text { (STAI) nachher }\end{array}$ & 8 & $\begin{array}{l}45,42 \\
38,57\end{array}$ & $\begin{array}{r}7,93 \\
12,32\end{array}$ & 311 \\
\hline $\begin{array}{l}\text { Lebenszufriedenheit Ehe und Partnerschaft } \\
\text { (FLZ) vorher } \\
\text { Lebenszufriedenheit Ehe und Partnerschaft } \\
\text { (FLZ) nachher }\end{array}$ & 8 & $\begin{array}{l}38,14 \\
37,85\end{array}$ & $\begin{array}{l}7,05 \\
5,75\end{array}$ & 783, \\
\hline
\end{tabular}

$\mathrm{N}=$ Anzahl der Probanden; MW=Mittelwert; SD=Standardabweichung 
Hinsichtlich der Zufriedenheit in der Partnerschaft fiel in der Gruppe der gesunden VPN der Kontrollbedingung ein signifikanter Unterschied beim Vergleich der beiden Testzeitpunkte auf. Bei der Messung der Zufriedenheit in der Partnerschaft, zeigte sich eine signifikante Verschlechterung im Vergleich zur ersten Untersuchung $(p<.05)$. In der 1 . Untersuchung wurde hier ein Mittelwert (MW) von 42,62 Punkten mit einer Standardabweichung (SD) von 4,26 Punkten und in der 2. Untersuchung ein signifikant niedrigerer Mittelwert (MW) von 40,75 Punkten mit einer Standardabweichung (SD) von 4,10 Punkten erreicht; (Abb. 23; Tab. 52).

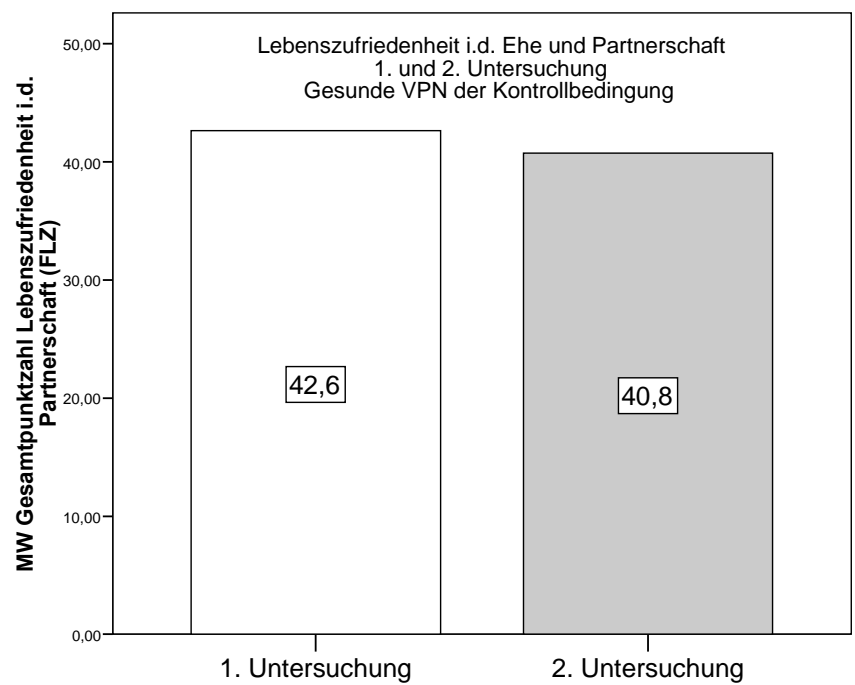

Abb. 23 Lebenszufriedenheit in der Ehe und Partnerschaft der gesunden VPN in der Kontrollgruppe in der 1. und 2. Untersuchung $(p=0,035)$ 
Tab.52 Mittelwerte der gesunden VPN in Kontrollbedingung, gemessen mit BDI, STAI, FLZ, in der 1. und 2. neuropsychologischen Untersuchung

\begin{tabular}{|l|r|r|r|r|}
\hline \multicolumn{1}{|c|}{$\begin{array}{l}\text { 1. und 2. neuropsychologische Untersuchung } \\
\text { Testverfahren }\end{array}$} & $\begin{array}{c}\text { Gesunde VPN } \\
\text { Kontrollgruppe } \\
\text { N }\end{array}$ & MW & SD & \multicolumn{1}{c|}{$\begin{array}{c}\text { Signifikanz } \\
\text { 2-seitig }\end{array}$} \\
\hline $\begin{array}{l}\text { Depressionsfragebogen } \\
\text { (BDI) vorher }\end{array}$ & 8 & 9,25 & 6,22 &, 190 \\
$\begin{array}{l}\text { Depressionsfragebogen } \\
\text { (BDI) nachher }\end{array}$ & 8 & 8,00 & 6,23 & \\
\hline $\begin{array}{l}\text { State Angst } \\
\text { (STAI) vorher }\end{array}$ & 8 & 36,75 & 8,06 & \\
$\begin{array}{l}\text { State Angst } \\
\text { (STAI) nachher }\end{array}$ & 8 & 35,12 & 10,26 &, 710 \\
\hline $\begin{array}{l}\text { Lebenszufriedenheit Ehe und Partnerschaft } \\
\text { (FLZ) vorher }\end{array}$ & 8 & 42,62 & 4,26 & \\
$\begin{array}{l}\text { Lebenszufriedenheit Ehe und Partnerschaft } \\
\text { (FLZ) nachher }\end{array}$ & 8 & & \\
\hline
\end{tabular}

$\mathrm{N}=$ Anzahl der Probanden; MW=Mittelwert; SD=Standardabweichung

Alle weiteren zur psychischen Befindlichkeit erhobenen Daten, wie Depressionsneigung und Ängstlichkeit, verschlechterten sich in der Gruppe der gesunden VPn in Kontrollbedingung jedoch nicht; 


\section{Diskussion}

\subsection{Interpretation der Ergebnisse}

Die vorliegende Studie diente der Überprüfung der Effektivität einer strukturierten viermonatigen neuropsychologisch-verhaltenstherapeutisch orientierten Gruppentherapie für Parkinson-Patienten (PD-Patienten) und deren gesunde Partner. Es sollte der Einfluss auf kognitive Leistungen, Depressionsneigung und Ängstlichkeit und Zufriedenheit in der Partnerschaft untersucht werden. Insgesamt nahmen 32 Versuchspersonen (VPN) an der Studie teil; davon wurden 16 VPN, die sich aus 8 PD-Patienten und deren gesunde Partner zusammensetzten, der Therapiebedingung zugeteilt. Weitere 16 VPN, ebenfalls 8 PD-Patienten sowie deren 8 gesunde Partner, stellten die Kontrollgruppe dar. Das Ausmaß der Erkrankung der PD-Patienten entsprach nach Hoehn und Yahr (1967) den Stadien 3-5, die den Bereich einer leichten bis mäßigen beidseitigen Erkrankung mit einer leichten Haltungsinstabilität, über eine stärkere Behinderung, wobei der Pat. noch ohne Hilfe laufen oder stehen kann, bis hin zur Notwendikeit der Nutzung eines Rollstuhls umfassten. Es wurde darauf geachtet, die Untersuchungen der PD-Patienten in einer "On-Phase“ durchzuführen. Weitere neurologische oder psychiatrische Erkrankungen konnten bei allen VPN durch eine ausführliche Anamneseerhebung unter besonderer Berücksichtigung neurologischer und psychiatrischer Vorerkrankungen bzw. eines gehäuften Auftretens entsprechender Erkrankungen in der Familie ausgeschlossen werden. Die Untersuchungen fanden sowohl für die Teilnehmer der Therapie als auch für die Kontrollgruppe zu Beginn und zum Ende der Therapie nach vier Monaten statt. Alle VPN mussten sich einer ausführlichen neuropsychologischen Testbatterie zur Einschätzung der intellektuellen Leistungsfähigkeit, der Aufmerksamkeit, der Konzentration, der Gedächtnisleistungen, der Exekutivfunktionen sowie der Beantwortung von Fragebögen zur psychischen Befindlichkeit und Zufriedenheit in der Partnerschaft unterziehen. 
Anhand eines in jeder Therapiestunde durchgeführten computergestützten Trainings sollten die kognitiven Leistungen der Teilnehmer gesteigert werden. Durch die Vermittlung von verhaltenstherapeutisch orientierten Methoden wurden positive Veränderungen im Sinne verminderter Depressionsneigung und Ängstlichkeit sowie gesteigerter Zufriedenheit in der Partnerschaft erwartet. Nachfolgend werden die Ergebnisse entsprechend der zuvor aufgestellten Hypothesen näher diskutiert.

\subsubsection{Verbesserung der Aufmerksamkeits- und Konzentrations- leistungen}

Naville (1922) hat als einer der ersten kognitive Dysfunktionen bei PD-Patienten näher erläutert. $\mathrm{Er}$ beschrieb ein Syndrom der Bradyphrenie, das durch reduzierte Aufmerksamkeit, Verlust an spontanem Interesse und Initiative, nachlassender Kapazität hinsichtlich Anstrengung sowie subjektivem und objektivem Ermüden gekennzeichnet ist. Eine „Bradyphrenie“ schien aber nicht nur bei PD-Patienten mit Demenz oder in Verbindung mit Apathie und Trägheit vorhanden zu sein. Nachfolgende Studien haben versucht die Frage zu beantworten, inwieweit eine allgemeine kognitive Verlangsamung beobachtbare kognitive Defizite von PD-Patienten beeinflußt. Die meisten Studien haben anhand einer Zeitmessung von der Aufgabenstellung bis zur Ausführung des offenen Verhaltens auf die vorhandene kognitive Schnelligkeit geschlossen, wobei deutlich gemacht werden sollte, dass die gesamte Reaktionsverlangsamung größer ist als die allein durch eine motorische Einschränkung erwartete Verlangsamung. In anderen Studien wurden unterschiedliche Aufgabenstellungen vorgegeben, die z.B. eine erhöhte kognitive Komplexitätsverarbeitung erfordern und so zu verlangsamten Reaktionszeiten führen können. Keine der Studien hat jedoch eine konsistente kognitive Verlangsamung nachweisen können (z.B. Gamsu, 1986; Horne, 1971; Rogers et al., 1987; Talland et al., 1964; Taylor et al., 1986; Wilson et al., 1980). 
Ein überdauerndes Muster, das erklären könnte, welche Patienten am ehesten betroffen sein könnten, wurde ebenfalls nicht gefunden.

In der vorliegenden Studie zeigte sich, dass sowohl die gesunden Probanden als auch die PD-Patienten, die an der Therapie teilnahmen, deutliche Leistungssteigerungen im Bereich der Aufmerksmkeit erzielen konnten. Die PDPatienten verbesserten sich signifikant in der basalen Aufmerksamkeit sowie in der Steigerungsfähigkeit der Aufmerksamkeit. Ferner konnten sie ihre Konzentrationsfähigkeit und kognitive Schnelligkeit tendenziell steigern. Die gesunden Partner der PD-Patienten, die regelmäßig an der Therapie teilgenommen haben, wiesen nach Beendigung der Therapie eine Verbesserung der Aufmerksamkeitsleistungen, der kognitiven Schnelligkeit sowie der Konzentrationsfähigkeit auf.

Demgegenüber wurde bei den PD-Patienten, die nicht an der Therapie teilnahmen, eine signifikante Verschlechterung in der basalen Aufmerksamkeit und der Konzentrationsfähigkeit beobachtet. Die Steigerungsfähigkeit der Aufmerksamkeit stellte sich in dieser Gruppe in der Nachuntersuchung als unverändert dar, jedoch erreichten sie wie die PD-Patienten der Therapiegruppen eine tendenzielle Verbesserung der kognitiven Schnelligkeit. Die Aufmerksamkeitsleistungen der gesunden Partner, die nicht an der Therapie teilnahmen, veränderten sich innerhalb von vier Monaten nicht signifikant, wobei sich die basale Aufmerksamkeit sowie die Steigerungsfähigkeit der Aufmerksamkeit tendenziell verschlechterte und die kognitive Schnelligkeit und Konzentration tendenziell verbessert werden konnte.

Beim Vergleich der Aufmerksamkeitsleistungen zwischen den PD-Patienten und den gesunden Partnern der Therapiegruppen zeigte sich, dass die Patienten insbesondere die zuvor signifikant schlechtere basale Aufmerksamkeitsfähigkeit deutlich steigern konnten, so dass nach Beendigung der Therapie zwischen den PD-Patienten und ihren gesunden Partnern keine weiteren signifikanten Unterschiede in sämtlichen getesteten Aufmerksamkeitsleistungen bestanden. In der Kontrollgruppe wurde eine gegensätzliche Entwicklung beobachtet. Nach 4 
Monaten stellten sich die zuvor nur tendenziell geringeren basalen Aufmerksamkeitsleistungen der PD-Patienten im Vergleich zu denen der gesunden Partner nun signifikant schlechter dar. In der Steigerungsfähigkeit der Aufmerksamkeit, der kognitiven Schnelligkeit und der Konzentrationsfähigkeit, unterschieden sich die Kontrollgruppen-PD-Patienten nicht weiter von ihren gesunden Partnern.

Bei der Interpretation der Ergebnisse sollte beachtet werden, dass viele Tests nur relativ einfache Aspekte kognitiver Funktionen einschätzen. Eine verlangsamte kognitive Verarbeitung bei PD-Patienten wird eher bei Aufgaben beobachtet, die größere Anforderungen an die kognitive Kapazität stellen. Bloxham und Mitarbeiter (1987) haben vorgeschlagen, dass einige Defizite in der Aufmerksamkeit und Reaktionsfähigkeit bei PD-Patienten durch eine Zunahme des „neuronalen Lärms“ aufgrund des Dopaminmangels erklärt werden können. Dies könnte wiederum Implikationen für die Verarbeitungsressourcen haben und zwar "nicht hinsichtlich ihres absoluten Niveaus, sondern hinsichtlich des Erreichens einer Signalstärke, die über das Neuronenrauschen im Hintergrund herausragt“.

PD-Patienten, die an der aktuellen Therapie teilgenommen haben, konnten ihre Aufmerksamkeitsleistungen also wahrscheinlich nicht nur durch das regelmäßige Training selbst, sondern u.a auch durch Gewöhnung und eine subjektiv wahrgenommene zunehmende Vereinfachung des Komplexitätsgrades (und folglich geringere Anforderung an ihre kognitive Kapazität) verbessern. Die vielberichtete „kognitive Verlangsamung“ stellt also nicht unbedingt eine allgemeingültige valide Beschreibung der beim Parkinson-Syndrom beobachteten charakteristischen kognitiven Dysfunktionen dar.

Ein Teil der kognitiven Störung beim Parkinson-Syndrom ist immer auch durch eine Beeinträchtigung der Aufmerksamkeits- und Konzentrationsfunktion bedingt. Nach Markowitsch (2002) befindet sich die Information noch im Kopf, aber die Anstrengung oder Eigeninitiative an diese heranzukommen, ist gestört. Dies 
spiegelt sich in den Ergebnissen der Eingangsuntersuchung beim Vergleich der PD-Patienten mit den gesunden Begleitpersonen wieder. Hieraus ergibt sich, dass eine Verbesserung der Aufmerksamkeitsfunktionen als basale Voraussetzung für eine Verbesserung der übrigen bei der PD-Erkrankung gestörten Leistungen, wie beispielsweise des Gedächtnisses, wesentlich ist.

Die hier vorgestellte Arbeit weist die Effektivität der angewandten Trainingsmethoden bei der Lösung dieses Problems nach. Trainierte PDPatienten konnten ihre Aufmerksamkeitsleistungen teilweise signifikant, in fast allen Bereich jedoch zumindest im Trend, verbessern. PD-Patienten unter Therapie konnten ihre Aufmerksamkeitsleistungen nahezu auf Werte gesunder Kontrollversuchspersonen verbessern.

\subsubsection{Steigerung der Gedächtnisleistungen}

Eine Anzahl von Studien hat in der Vergangenheit gezeigt, dass verschiedene Gedächtnisaspekte unterschiedlich stark bei Patienten mit einem ParkinsonSyndrom beeinträchtigt sind, wobei einige Gedächtnisprozesse einen auffälligen Verlust aufweisen und andere gänzlich oder teilweise intakt bleiben (z.B. Freedman et al., 1984; Litvan et al., 1991; Massman et al.,1990). Hinsichtlich der Leistung bei Aufgaben zur verbalen Merkspanne zeigten PD-Patienten z.B. nur ein leichtes Defizit, jedoch größere Abweichungen und Defizite im verbalen bzw. non-verbalen Kurzzeitgedächtnis.

In der vorliegenden Studie konnten die PD-Patienten, die an dem viermonatigen neuropsychologischen Training teilnahmen im Gegensatz zu den PD-Patienten der Kontrollgruppen, ihr visuelles Kurzzeitgedächtnis sowie das modalitätsübergreifende Langzeitgedächtnis signifikant steigern. Weitere leicht tendenziell verbesserte Leistungen umfassten die verbale und non-verbale Merkspanne und das verbale 
Kurzzeitgedächtnis. Die Leistungssteigerung war der der gesunden Probanden, die an der Therapie teilnahmen, ähnlich. Diese erzielten insgesamt Verbesserungen ihrer Gedächtnisleistungen. Signifikante Leistungssteigerungen erreichten sie insbesondere im modalitätsübergreifenden Kurz- und Langzeitgedächtnis. Aber auch die verbale und visuelle Merkspanne konnten hier tendenziell verbessert werden.

PD-Patienten, die nicht an der Therapie teilgenommen haben, zeigten nach 4 Monaten tendenziell schlechtere Leistungen sowohl in der verbalen und visuellen Merkspanne, als auch im Langzeitgedächtnis und verbalen Kurzzeitgedächtnis; die Leistung des non-verbalen Kurzzeitgedächtnisses zeigte sich tendenziell, jedoch nicht signifikant wie bei den PD-Patienten der Therapiegruppe, verbessert. Die gesunden Probanden der Kontrollgruppe erzielten außer in der verbalen Merkspanne, die als diskret vermindert erschien, tendenzielle Leistungssteigerungen in der visuellen Merkspanne, dem verbalen und nonverbalen Kurzzeitgedächtnis sowie dem Langzeitgedächtnis.

Der Vergleich der Leistungen im Gedächtnisbereich zwischen den PD-Patienten und den gesunden Partnern ergab für die Therapiegruppen nach 4 Monaten insgesamt keine signifikanten Unterschiede, wobei jedoch anzumerken ist, dass die PD-Patienten tendenziell weiterhin unter den erreichten Mittelwerten der gesunden Partner lagen.

Wenn man die Gedächtnisleistungen der PD-Patienten und die der gesunden Partner der Kontrollgruppe betrachtet zeigt sich, dass die Leistungen der PDPatienten im Vergleich zu den gesunden Partnern innerhalb von 4 Monaten nicht signifikant, jedoch deutlicher als die der PD-Patienten in Therapiebedingung, abgenommen haben. 
Nach Zakzanis (1999) scheint die Leistungsfähigkeit im Langzeitgedächtnisbereich bei nicht dementen PD-Patienten sehr heterogen zu sein, wobei PD-Patienten sich entweder stark oder gar nicht von gesunden Probanden unterscheiden. Gedächtnisdefizite bei Parkinson sind It. Meinung des Autors möglicherweise eher mit ineffizienten Abrufstrategien assoziiert als mit einer beeinträchtigten Einspeicherung und Konsolidierung, wie es z.B. bei der Alzheimer-Demenz der Fall ist (Freedman, 1990b; Mahurin et al., 1993; Soukup \& Adams, 1996).

Andere Untersuchungen, z.B. Dubois und Kollegen (1990), wiesen bei über 60jährigen PD-Patienten den normalen Alterungsprozess deutlich übersteigende schlechtere Gedächtnisleistungen nach als bei den gesunden Kontrollprobanden. Bei PD-Patienten mit einem früheren Beginn der Erkrankung, d.h. bei einem Beginn unter 40 Jahren, zeigten sich nur leichte Gedächtnisdefizite, die sich auf Aufgaben mit komplexeren Anforderungen beschränkten. Obwohl das ParkinsonSyndrom durch eine massive Beeinträchtigung der dopaminergen nigrostriatalen Bahnen und nur geringe Störungen anderer neuronaler Systeme charakterisiert ist, sind kognitive Defizite bei PD-Patienten mit frühem Beginn trotz der Ausprägung der dopaminergen nigrostriatalen Läsionen nur gering, so dass dieser Aspekt wenig zur den kognitiven Veränderungen beim ParkinsonSyndrom beiträgt. Die Autoren argumentieren, dass bei älteren PD-Patienten zusätzlich hinzukommende Hirnläsionen als Ursache für die ausgeprägteren Gedächtnisdefizite in Frage kommen könnten. So werden Defizite aufgrund neuronaler Degeneration deutlicher erkennbar.

Andere Untersuchungen, z.B. von Gibb und Lees (1988), haben jedoch gezeigt, dass es keinen pathologischen Unterschied zwischen PD-Patienten mit frühem vs. spätem Beginn der Erkrankung zu geben scheint. Defizite beim freien Abruf von Gedächtnisinhalten treten nach Flowers und Kollegen (1984) im Rahmen von Aufgaben auf, die einen aktiveren (im Gegensatz zu passiver einfacher Wiedererkennung) kognitiven Einsatz an das Individuum erfordern. 
Auch Weingartner und Mitarbeiter (1984) kamen zu dem Entschluss, dass man zwischen automatischen und Anstrengung erfordernden kognitiven Prozessen unterscheiden müsse. Aufgaben, wie der freie Abruf aus dem Gedächtnis, setzen Fähigkeiten, wie die Aufrechterhaltung von Konzentration und Ausschöpfung von Ressourcen, sowie eine gewisse kognitive Kapazität voraus. Die Autoren zeigten, dass PD-Patienten bei solchen Aufgaben am deutlichsten beeinträchtigt waren, die am meisten Anstrengung erforderten.

Die positiven Ergebnisse der aktuellen Studie könnten in dem Sinne interpretiert werden, dass die Patienten der Therapiegruppen im Gegensatz zu den Patienten der Kontrollgruppe, schon allein durch das regelmäßige Gedächtnistraining und aufgrund der Gewöhnung an die Trainings- bzw. Testsituation weniger Anstrengung für den freien Abruf von Gedächtnisinhalten benötigten und so ihre Gedächtnisleistung steigern konnten.

Wahrscheinlich haben darüber hinaus auch das Wissen, an einer Studie teilzunehmen, "wichtig zu sein" und das positive Feedback im Therapiesetting, die Motivation der an der Therapie teilnehmenden Patienten beeinflußt. Im Rahmen der Gruppentherapie lernten die PD-Patienten, dass auch die anderen Teilnehmer, teilweise auch die gesunden Probanden, ähnliche Probleme haben, was sich durchaus positiv auf die Akzpetanz und Einsicht in die kognitiven Defizite auswirkte. Durch die Beobachtung der anderen Therapieteilnehmer und deren Strategien zur Verbesserung der Gedächtnisleistungen wurde der eigene Einsatz entsprechender Strategien gesteigert.

Wenn man davon ausgeht, dass das regelmäßig angebotene Gedächtnistraining die Entwicklung neuer kognitiver Strategien allgemein unterstützt und durch eine erhöhte Anforderung an die kognitive Aktivität einen positiven Einfluss auf die Gedächtnisfunktionen bewirkt, so könnte nach den vorliegenden Ergebnissen der aktuellen Studie durchaus noch ein „kognitives Entwicklungspotential“ bei PD- 
Patienten vermutet werden. Das heißt, dass auch bei einer bereits eingeschränkten Reservekapazität, die als Voraussetzung für eine Verbesserung der kognitiven Leistungsfähigkeit angesehen wird (Kühl \& Baltes, 1989), eine deutliche Leistungssteigerung zu erzielen ist.

Die bei der PD-Krankheit auftretende kognitive Störung äußert sich im Alltag häufig zuerst als Störung der Aufmerksamkeit und der exekutiven Funktionen. Erst mit Fortschreiten der Erkrankung treten Beeinträchtigungen der Gedächtnisfunktionen hinzu. Die in dieser Studie untersuchte Gruppe von PDPatienten spiegelt dies wider. Sie zeigte im Vergleich zu gesunden VPN deutliche Minderleistungen im Bereich der Aufmerksamkeits- und Exekutivfunktionen. Die untersuchten PD-Patienten der Stadien 3-5 nach Hoehn und Yahr (1967) hatten aber auch bereits vor Studienbeginn weniger gute Gedächtnisleistungen als die gesunden Kontrollpersonen.

Dies betraf fast alle untersuchten Gedächtnisbereiche. Die in der vorliegenden Studie angewandten Trainingsinstrumente führten teilweise signifikant, in allen übrigen geprüften Gedächtnisbereichen jedoch auch im Trend, zu einer Verbesserung der Leistungen, während die nicht trainierte Gruppe der PDPatienten vermutlich entsprechend dem natürlichen Verlauf der Erkrankung im Untersuchungszeitraum eine Verschlechterung ihrer Gedächtnisleistungen zeigte.

Gedächtnisfunktionen sollten nicht unabhängig von anderen kognitiven Leistungen, wie den Exekutiv- und Aufmerksamkeitsfunktionen betrachtet werden. Insofern müssen die Ergebnisse in den Abschnitten Aufmerksamkeit und Exekutivfunktionen im Zusammenhang mit den Ergebnissen im Gedächtnisbereich gesehen werden und haben möglicherweise einen nicht unwesentlichen Anteil hieran. 
Letztlich ist jedoch davon auszugehen, dass Funktionsverbesserungen in allen drei Bereichen zu einer gegenseitigen Potenzierung im Trainingsablauf führen, was auch den Gedächtnisleistungen zugute kommen sollte. Wahrscheinlich werden diese Funktionen darüber hinaus auch durch Verbesserungen der Befindlichkeit aufgrund neu erlernter Coping-Strategien im Umgang mit der Erkrankung unterstützt.

\subsubsection{Verbesserung der exekutiven Funktionen}

Bei der Einschränkung exekutiver Funktionen sind meist Läsionen im Frontallappenbereich beteiligt (Damasio \& Anderson, 1993; Robinson et al., 1980; Woods \& Troster, 2003). Man geht davon aus, dass die Ausführung exekutiver Funktionen von einer halbgeschlossenen Schleife abhängig ist, deren Projektionen aus den kortikalen Assoziationsbereichen in und durch den Nucleus Caudatus und dann zurück durch thalamo-kortikale Bereiche in den präfrontalen Kortex fließen (z.B. Alexander et al., 1986; DeLong \& Georgopoulos, 1981). Da beim Parkinson-Syndrom durch einen Dopaminmangel das nigrostriatale System beeinflußt wird, könnten negative Konsequenzen für solche kognitiven Fähigkeiten die Folge sein, die auf diese komplexe Schleife angewiesen sind. In der Literatur finden sich viele Hinweise auf Defizite der exekutiven Funktionen bei Patienten mit Parkinson-Syndrom (z.B. Brown \& Marsden, 1990).

Insbesondere das planerische Handeln und die kognitive Flexibilität scheinen für PD-Patienten aufgrund andauernder „perseverativer Intrusionen“ (Drewe, 1974; Milner, 1964; Nelson, 1976) besonders schwierig durchführbar zu sein. Viele der Patienten gaben zu erkennen, dass sie zwar die konzeptuellen Anforderungen der Aufgabe verstanden haben, jedoch nicht in der Lage waren, diese Information zur Lenkung ihrer Vorgehensweise zu nutzen. Andere Patienten 
erkannten, dass sie Fehler gemacht haben, konnten diese aber scheinbar nicht korrigieren. Milner (1964) hat aus diesem Grund vorgeschlagen, dass die Probleme von PD-Patienten bei der Ausführung dieser Tests als „allgemeine Unfähigkeit, ein Antwortverhalten entsprechend der sich verändernden Anforderungen aus der Umwelt schnell anzupassen" interpretiert werden sollten und weniger als eine Störung des abstrakten Denkens.

Lees und Smith (1983) zeigten anhand einer Studie, dass in den frühen Stadien des Parkinson-Syndroms diskrete Schwierigkeiten mit einem zunehmenden Prozentsatz perseverativer Fehler bei der Fertigstellung von Konzepten bestehen. Die Autoren führen dies nicht auf eine reduzierte intellektuelle Leistungsfähigkeit oder auf Depressions-, Medikations- bzw. Alterseffekte zurück. Sie sind der Meinung, dass die internale bzw. externale Aufmerksamkeitskontrolle eine wichtige Rolle spielt. Auch Brown und Marsden (1988) gehen davon aus, dass PD-Patienten insbesondere bei Aufgaben beeinträchtigt sind, bei denen sie sich auf interne Strategien verlassen müssen und weniger bei der Bearbeitung von Aufgaben, die externale Hinweisreise bieten. Diese Unterscheidung kann nach Überzeugung der Autoren auch auf andere Aufgabenstellungen übertragen werden, die eine internale Kontrolle erfordern, wie z.B. der freie Gedächtnisabruf oder eine externale Hilfestellung geben, wie z.B. bei Wiedererkennungsaufgaben. Taylor und Kollegen (1986) kommen in ihrer Studie, in der sie PD-Patienten verschiedene Aufgabenstellungen vorgaben, zu einem ähnlichen Resultat. Ihre Patienten waren nur in dem Teil der Aufgabenbearbeitung beeinträchtigt, die eine „spontane Generierung aufgabenspezifischer Planung" erforderte. Sie gehen davon aus, dass das Vorhandensein einer beeinträchtigten Leistung von dem für einen Erfolg notwendigen Niveau internaler subjektiv organisierter Leitlinien abhängt. Die PD-Patienten erreichten nur adäquate Leistungen in Aufgabenstellungen, in denen das Material bei der Darbietung bereits organisiert war. 
Weingartner und Kollegen (1984) sowie Ransmayer und Mitarbeiter (1987) zogen die gleichen Rückschlüsse, wobei auch hier von den Autoren unterschieden wird zwischen einerseits internalen, aktiven und Anstrengung erfordernden Aufgaben und andererseits passiven, automatisierten Aufgaben, bei denen externale Hinweise gegeben werden oder bei denen die Stimuli während der Darbietung bereits organisiert werden.

In der vorliegenden Studie wiesen die PD-Patienten, die an der Therapie teilnahmen, nach Beendigung der Therapie tendenzielle Leistungsverbesserungen des verbalen und non-verbalen Arbeitsgedächtnisses, der kognitiven Flexibilität und des planerischen Handelns auf, wobei sich insbesondere die zuvor deutlich reduzierte Fähigkeit des planerischen Handelns nach Beendigung der Therapie der Leistungsfähigkeit der gesunden Partner annäherte. Die Leistungssteigerungen konnten zwar nicht als signifikant eingestuft werden wie die der gesunden Probanden, die an der Therapie teilnahmen, sie waren jedoch deutlich besser als die der PD-Patienten in der Kontrollgruppe zu bewerten. Diese zeigten eine gegensätzliche Entwicklung mit signifikanten Leistungseinbußen im Bereich der exekutiven Funktionen. Hiervon betroffen waren insbesondere das verbale und non-verbale Arbeitsgedächtnis und die kognitive Flexibilität. Die Leistungsfähigkeit der exekutiven Funktionen der gesunden Teilnehmer in der Kontrollgruppe hat sich nach vier Monaten nicht signifikant verändert. Auch der Vergleich zwischen den exekutiven Funktionen der PD-Patienten und denen der gesunden Partner der Kontrollgruppe zeigte sowohl in der ersten als auch in der zweiten Untersuchung nach vier Monaten keine Veränderung der Leistungsunterschiede mit einer persistierenden und tendenziell geminderten Leistungsfähigkeit der PD-Patienten, was als mögliche Folge der Unfähigkeit, mentale Strategien effizient auszuwählen, zu interpretieren ist.

Da gut erhaltene exekutive Funktionen u.a. für die adäquate Bewältigung neuer Situationen von Bedeutung sind, könnte eine mögliche Erklärung der o.g. verbesserten Ergebnisse der PD-Patienten der Therapiegruppen in der 2. 
Testung lauten, dass durch die lange Therapiezeit der Aspekt der Neuheit der Aufgabenstellungen immer geringer geworden ist, so dass die Patienten der Therapiegruppen durch den Gewöhnungseffekt der Trainings- und Testsituationen nach vier Monaten tendenziell bessere Ergebnisse erreichen konnten als die Patienten der Kontrollgruppe. Aber auch die Einbindung der Bezugspersonen sowie der Vorteil, Vergleichsmöglichkeiten mit anderen Patienten der Therapiegruppe zu haben, könnte einen positiven Effekt darstellen.

Nachdem den Patienten verdeutlicht werden konnte, dass Leistungsdefizite z.B. beim planerischen Handeln bestanden, lernten sie in der Therapie einige psychotherapeutische Bausteine, wie z.B. die Planung und Durchführung zukünftiger Aktivitäten, zumindest teilweise praktisch umzusetzen. Aber auch andere Fähigkeiten aus dem Bereich der Exekutivfunktionen, wie die Befindlichkeiten anderer Menschen einzuschätzen sowie die Fähigkeit, selbständig eine angemessene Wortwahl zu treffen, Bewertungen vornehmen zu können, Impulse zu kontrollieren etc, sind für eine erfolgreiche soziale Interaktion von Bedeutung und wurden im Rahmen der Therapie gefördert.

Schließlich ist in diesem Zusammenhang noch anzumerken, dass die Behandlung eines Defizits, dass dem Patienten weniger bewußt ist (wie eine Störung der Exekutivfunktionen), immer schwieriger ist als eines, dass ihm bewußt ist (z.B. nachlassende Gedächtnisleistungen), so dass die lediglich „tendenziellen“ Verbesserungen der PD-Patienten, die an der Therapie teilnahmen, auch hierdurch teilweise erklärt werden könnten.

\subsubsection{Abnahme depressiver Tendenzen und Ängstlichkeit}

Eine erhöhte Ängstlichkeit ist bei PD-Patienten häufig zu beobachten und kann zu erheblichem Streß führen. Angst ist meist ein Symptom depressiver Störungen und kann manchmal sogar das vorherrschende Symptom sein. Die zugrundeliegende Depression kann deshalb schnell übersehen werden (Hurwitz \& Calne, 2001). 
In der vorliegenden Studie konnten sowohl depressive Tendenzen als auch Ängstlichkeit (State-Angst) in der Gruppe der PD-Patienten, die an der Therapie teilnahmen, nur tendenziell verringert werden. Auch die gesunden Partner, die an der Therapie teilnahmen, wiesen nach vier Monaten eine tendenziell geringere Depressionsneigung und State-Angst auf. Im Gegensatz dazu stiegen Depressionsneigung und State-Angst bei den Patienten aus der Kontrollgruppe tendenziell an. Die gesunden Probanden der Kontrollgruppe zeigten in der zweiten Untersuchung nach vier Monaten eine tendenziell geringere Depressionsneigung und Ängstlichkeit. Der Vergleich der PD-Patienten und ihrer gesunden Partner der Therapiegruppe bzgl. Depressionsneigung zeigt, dass die Patienten weiterhin tendenziell höhere Depressions- und Ängstlichkeitswerte aufwiesen als ihre gesunden Partner. Auch in der Kontrollgruppe zeigte sich, dass die PD-Patienten nach vier Monaten eine tendenziell höhere Depressionsneigung und Ängstlichkeit aufwiesen als ihre gesunden Partner.

Zunehmende Ängstlichkeit kann sich auf die Angehörigen von PD-Patienten übertragen und zu einer erheblichen Belastung führen. Ein entsprechender psychotherapeutischer Ansatz sollte die Patienten und Angehörigen der Therapiegruppen darüber informieren, dass ein Zusammenhang zwischen der Angst vor einer Verschlechterung der Symptome und den „Off-Zeiten“ besteht.

Aber auch eine Depression kann sowohl für den Patienten als auch für die Angehörigen so schwerwiegend sein wie eine körperliche Behinderung (Aarsland et al., 1996). Depressionen interferieren aufgrund nachlassender Initiative und Kooperation seitens des Patienten häufig mit dessen Betreuung. Unbehandelte Depressionen verhindern eine bestmögliche Wirkung der Medikation und stören Familienbeziehungen. In der aktuellen Studie neigten die gesunden Partner der PD-Patienten jedoch nur zu geringen depressiven Tendenzen und Ängstlichkeit. Andere Untersuchungen haben jedoch gezeigt, dass die Betreuer von PDPatienten häufig unter Überforderung, Stress und Depressionen leiden (z.B. Secker \& Brown, 2005). Die Autoren konnten nachweisen, dass die 
Durchführung von 14 Sitzungen kognitiver Verhaltenstherapie bei Betreuern von PD-Patienten zu einer signifikanten Reduzierung der empfundenen Stressbelastung führte. In einer Meta-Analyse haben Cole und Vaughan (2005) festgestellt, das eine kognitive Verhaltenstherapie bei PD-Patienten die angemessenste Behandlung von Depressionen ist. Die mittels der verhaltenstherapeutisch orientierten Methoden, wie z.B. durch Entspannung, kognitive Umstrukturierung und Stressbewältigungstraining neu erhaltene Kontrolle, stellte für die Patienten eine neue Erfahrung dar, da das Gefühl von Hiflosigkeit, Depression und Ängstlichkeit tendenziell verbessert werden konnte.

Die Beherrschung von angemessenen Verhaltensstrategien kann das pathologische Fortschreiten möglicherweise verringern und dem Entstehen von Hilflosigkeit und Frustration entgegenwirken und u.a. auch die kognitive Leistungsfähigkeit verbessern. Der Übertragung dieser Bewältigungsstrategien auf den Altag sind jedoch Grenzen gesetzt. Über Jahrzehnte trainierte Reaktionen können sicherlich nicht einfach modifiziert werden.

Bei Patienten mit Parkinson-Syndrom zeigen sich schnell die Grenzen einer ambulanten gruppenpsychotherapeutischen Behandlung. Aufgrund des progredienten Charakters der Krankheit besteht eine gewisse Therapieresistenz. Für viele Symptome, die einer Depression zugrunde liegen, ist wahrscheinlich ein höheres Maß an Training erforderlich, das auch nach Abschluss der Therapie beibehalten werden sollte. Ein weiterer einen signifikanten Therapieerfolg hinsichtlich Depression und Ängstlichkeit verhindernder Faktor könnte der sekundäre Krankheitsgewinn sein, den manche Patienen nicht aufgeben wollen. Jedoch ist das Auftreten von Depressionen bei PD-Patienten nicht nur abhängig von den zur Verfügung stehenden persönlichen und sozialen Bewältigungsstrategien, sondern auch von der Art und Schwere der strukturellen Beeinträchtigung, dem Ausmaß des Verlusts an Transmittern, der funktionellen Reserve und Plastizität des ZNS. 


\subsubsection{Zunahme der Zufriedenheit in der Partnerschaft}

In einer Untersuchung von Martinez und Mitarbeitern (2005) wurde bereits berichtet, dass die Fähigkeit von PD-Patienten, ihren Alltag möglichst selbständig bewältigen zu können, mit der Lebenszufriedenheit ihrer Betreuer positiv korrelierte. Betrachtet man den psycho-sozialen Bereich, leiden PD-Patienten unter einer weit über die motorische Behinderung hinausgehenden Beeinträchtigung. Selbst wenn die sozialen Rollen noch ausgefüllt werden können, führen die willkürlich nur schwer kontrollierbaren Symptome vielfach zu einem geminderten Selbstwertgefühl und einer verstärkten Neigung, öffentliche Situationen zu meiden. Werden diese Rückzugstendenzen dann durch unumgängliche Alltagshandlungen durchbrochen, fehlen die sozialen Kompetenzen im angemessenen Umgang mit der Behinderung.

Auch Leplow und Kollegen (1993) kamen nach Durchführung ihres verhaltenstherapeutischen Gruppenprogramms bei PD-Patienten zu ähnlichen Schlussfolgerungen. Die Autoren gingen davon aus, dass auch ältere Patienten von einem strukturierten, situationsspezifischen Vorgehen profitieren können. Durch die Symptome, die sich bei emotionaler Belastung verstärken, werden höhere Anforderungen an die soziale Kompetenz gestellt als sie ein nicht behinderter Mensch benötigt, daher ist der Erwerb von spezifischen sozialen Fertigkeiten in jedem Fall ein wichtiger Bestandteil, der den Patienten mehr Aktivitätsspielraum bringt.

In der vorgestellten Therapie war es für die Patienten und ihre Lebenspartner wichtig zu lernen, dass die z.B. die mit dem Tremor verbundenen unangenehmen emotionalen Begleitumstände nicht zum Auslöser für Vermeidungsreaktionen werden. Die geschützte Gruppenatmosphäre bot den Teilnehmern auch die Gelegenheit, alternative, aktive Stressbewältigungsstrategien auszuprobieren und anschließend in die Realsituation umzusetzen. Die PD-Patienten, die an der Therapie teilnahmen, berichteten nach Beendigung der Therapie tatsächlich über eine tendenziell 
größere Zufriedenheit in der Ehe. Aber auch die Zufriedenheit der gesunden Partner nahm nicht weiter ab. Demgegenüber wiesen die gesunden Probanden der Kontrollgruppe eine signifikant geringere Zufriedenheit in der Ehe und Partnerschaft auf. Bei den PD-Patienten der Kontrollgruppe ist der Stand der Zufriedenheit in der Ehe bzw. Partnerschaft über 4 Monate konstant geblieben. 


\subsection{Allgemeine Diskussion}

Insgesamt wirkte sich die Teilnahme der PD-Patienten an einer neuropsychologisch-verhaltenstherapeutisch orientierten Therapie im Sinne der vor Studienbeginn o.g. aufgeführten Hypothesen positiv auf ihre kognitiven Leistungen und emotionale Befindlichkeit aus. Die Mehrzahl der geprüften kognitiven Parameter verbesserte sich tendenziell. Insbesondere die Aufmerksamkeit und Leistungsfähigkeit des visuellen Gedächtnisses konnten signifikant gesteigert werden. Demgegenüber musste für die Kontrollgruppe bei einigen Parametern eine Stagnation, bei zahlreichen anderen eine Verschlechterung festgestellt werden.

Im Gegensatz zu den berichteten Ergebnissen der vorliegenden Studie wird in der Literatur meist von Defiziten berichtet, die mit dem Fortschreiten der Erkrankung zunehmen, angefangen von einer leichten neuropsychologischen Beeinträchtigung bis hin $z u$ ausgeprägten kognitiven Veränderungen. Nachlassende Gedächtnisleistungen bei nicht-dementen PD-Patienten werden insbesondere mit einem Defizit des fronto-subkortikalen Systems assoziiert, welche zu Störungen in der exekutiven Kontrolle des Aufmerksamkeitssystems führt, die den Enkodierungsprozess für Kurz- und Langzeitgedächtnisleistungen einschränken, d.h. dass ein Teil der kognitiven Störung beim PD-Syndrom immer auch auf eine Beeinträchtigung der Aufmerksamkeits- und Konzentrationsfunktion zurückzuführen ist.

Mit zunehmender Dauer der Erkrankung und progressivem Dopaminmangel in der Substantia nigra wird der funktionale Zusammenhalt neuronaler Kreise, die die Basalganglien und das Frontalhirn miteinander verbinden gestört und Leistungen, wie kognitive Flexibilität und Abstraktionsfähigkeit sind deutlicher reduziert, wenn bereits größere Defizite bei der Gedächtniskonsolidierung und des Langzeitgedächtnisses bestehen. 
Die Forschung über Ursachen kognitiver Defizite bei PD-Patienten hat aber auch zu anderen Ansätzen geführt, wie z.B. der Untersuchung zugrundeliegender psychologischer Prozesse. Nicht erkannte Depressionen beim ParkinsonSyndrom können z.B. fälschlicherweise dazu führen, dass Patienten als „kognitiv eingeschränkt“ bzw. „dement“ diagnostiziert werden. Wenn eine Depression unerkannt bleibt und nicht behandelt wird, kann dies zu einer übertrieben pessimistischen Einschätzung kognitiver Kapazitäten führen (z.B. Norman et al., 2002; Starkstein et al.,1990; Taylor et al., 1987; Troster et al., 1995). Typischerweise wird jedoch eine gewisse Stimmungslabilität oder eine leichte Form von Dysthymie erwartet. Aber auch Symptome einer Zwangsstörung, wie z.B. die übertriebene Sorge über einzelne Details oder Sauberkeit, können sich bei Patienten mit einer Basalgangliendysfunktion manifestieren. Darüber hinaus wurden Persönlichkeitseigenschaften, wie Rigidität, Introversion, Ordentlichkeit und übertriebene Ernsthaftigkeit, sowohl prämorbid als auch bei Patienten, bei denen bereits ein Parkinson-Syndrom diagnostiziert wurde, beobachtet (z.B Cote, 1999; Glosser et al., 1995; Heberlein et al., 1998; Mega \& Cummings; 1994).

Durch die Interaktion somatischer und psychologischer Faktoren können soziale Angst und eine stressbedingte Zunahme der Parkinson-Symptome entstehen. PD-Patienten befürchten häufig, in der Öffentlichkeit negativ beurteilt zu werden. Ein zunehmender sozialer Rückzug ist oft die Folge. Die sekundären psychologischen Symptome im Rahmen einer Parkinson-Erkrankung sollten deshalb auch berücksichtigt werden, damit sie zumindest teilweise psychotherapeutisch behandelt werden können. Aus diesem Grund sollten einige der wichtigsten Strategien, wie Entspannungstraining, kognitive Umstrukturierung und ein speziell auf die Erkrankung bezogenes Training sozialer Fertigkeiten immer auch in die medikamentöse Behandlung miteinbezogen werden.

Auch (Ehe-)Partner, Verwandte und Freunde von Patienten mit ParkinsonSyndrom müssen lernen, mit Symptomen, die die soziale Interaktion stark beeinflussen, umzugehen. Psychologische Hilfe wird hier dringend benötigt und 
zwar nicht nur für die Patienten, sondern auch für den Erhalt der Gesundheit inrer Angehörigen. Partner, Verwandte und Freunde brauchen eine systematische informative Unterstützung hinsichtlich der psychologischen Aspekte der Erkrankung sowie eine systematische Schulung, um angemessen mit schwierigen Situationen, die unvermeidlich bei der Betreuung eines Patienten mit Parkinson-Syndrom auftreten, umgehen zu können. Das Ziel dieser Maßnahmen ist es, PD-Patienten dabei zu helfen, den positiven Effekt ihrer Medikation besser auszuschöpfen und bekannte negative Effekte sozialer und emotionaler Stressoren, entgegenzuwirken. Nur wenige Erkrankungen zeigen eine derart intensive und direkte Interaktion zwischen psychologischen Faktoren und dem Schweregrad neurologischer Symptome wie das Parkinson-Syndrom. Genau wie die Medikation in den frühen Stadien der Erkrankung am effektivsten ist, sollte auch die psychologische Intervention während dieses Stadiums eingesetzt werden, so dass zumindest einige der trotz Medikation weiterbestehenden psychologischen Probleme erfolgreich behandelt werden können. 


\subsection{Konsequenzen aus der vorliegenden Studie}

Die aus der vorliegenden Arbeit resultierenden Ergebnisse machen deutlich, dass häufig berichtete kognitive Defizite bei Patienten mit Parkinson-Syndrom nicht zwangsläufig mit dem Fortschreiten der Erkrankung zunehmen und nicht nur organischer Ursache sind sondern vielmehr, dass einige dieser Defizite durch eine entsprechende neuropsychologische bzw. verhaltenstherapeutisch orientierte Therapie verbessert werden können. Aufgrund der geringen Zahl der Versuchspersonen sind diese Daten trotz der zunächst ermutigenden Ergebnisse unter Vorbehalt zu sehen. Eine Fortführung der Therapie zur weiteren Datenerhebung scheint sinnvoll. Aus dem Vergleich der gegensätzlichen Entwicklung der Patienten mit und ohne Therapie kann jedoch bereits jetzt schon geschlossen werden, dass das hier vorgestellte neuropsychologischverhaltenstherapeutische Programm als effektives Therapiemodul den Therapieblöcken Medikation und Physiotherapie hinzugefügt werden sollte. Dies trifft insbesondere für die in diesem Projekt untersuchten PD-Patienten in mittlerem bis fortgeschrittenem Stadium zu. Für diese Patienten ist es von besonderer Wichtigkeit, primär offensichtlich beeinträchtigte motorische Fähigkeiten zu erhalten bzw. zu verbessern. Diese Fähigkeiten können aber nur optimal genutzt werden, wenn hierzu auch die kognitiven Voraussetzungen bestehen.

Schließlich sollte noch einmal darauf hingewiesen werden, dass das ParkinsonSyndrom ein komplexes multidimensionales System darstellt, das sich sowohl inter- als auch intraindividuell unterschiedlich darstellen kann. Es ist wahrscheinlich, dass weder rein psychologische noch ausschließlich neurobiologische Faktoren ausreichen, um das Vorhandensein kognitiver Beeinträchtigungen im Rahmen eines Parkinson-Syndroms zu erklären.

Aufrund der vorliegenden Ergebnisse sollten Betreuungsprogramme, die an einem schwierigen, älteren, teilweise ängstlichen und kognitiv verlangsamten Klientel mit starken motorischen Beeinträchtigungen durchgeführt werden, 
entsprechend erweitert werden. Weitere Vorgehensweisen von Interventionsprogrammen müssten insbesondere die Frage der langfristigen Sicherung der Effekte berücksichtigen. Für neuropsychologischverhaltenstherapeutisch orientierte Therapieprogramme bedeutet dies, dass sich die Evaluationsstrategien an den immer wieder neu zu formulierenden kognitiven und psychischen Anpassungsnotwendigkeiten orientieren müssen. 


\section{Zusammenfassung}

Nach Beendigung einer viermonatigen neuropsychologischverhaltenstherapeutisch orientierten Gruppentherapie für Parkinson-Patienten (PD-Patienten) und deren gesunde Partner, erreichten die PD-Patienten der Therapiegruppen signifikante Verbesserungen im Bereich der basalen Aufmerksamkeitsleistungen $(p=0,02)$ sowie in der Steigerungsfähigkeit der Aufmerksamkeit $(p=0,04)$. PD-Patienten, die nicht an der Therapie teilnahmen (Kontrollgruppe), konnten sich im Vergleich zur Voruntersuchung lediglich tendenziell in der kognitiven Schnelligkeit verbessern. Die Leistungen in der Aufgabe zur basalen Aufmerksamkeit verschlechterten sich in dieser Gruppe im Verlauf signifikant $(p=0,04)$ und auch die Konzentrationsfähigkeit ließ deutlich nach $(p=0,02)$. Die Steigerungsfähigkeit der Aufmerksamkeit stellte sich in der Kontrollgruppe der Nachuntersuchung als unverändert dar.

Die PD-Patienten der Therapiegruppen konnten ferner die Leistungsfähigkeit ihres non-verbalen Kurzzeitgedächtnisses signifikant steigern $(p=0,01)$. Andere untersuchte kognitive Funktionen, wie verbales Arbeits-, Kurz- und Langzeitgedächtnis sowie die kognitive Flexibilität verbesserten sich in dieser Gruppe tendenziell. Die PD-Patienten der Kontrollgruppe lieferten nach 4 Monaten tendenziell schlechtere Leistungen, sowohl hinsichtlich der modalitätsübergreifenden Merkspanne und des Langzeitgedächtnisses sowie des verbalen Kurzeitgedächtnisses, als aber auch im Bereich der Exekutivfunktionen, wie verbalem und non-verbalem Arbeitsgedächtnis und der kognitiven Flexibilität.

Depressive Tendenzen und Ängstlichkeit konnten in der Gruppe der PDPatienten, die an der Therapie teilnahmen, tendenziell verringert werden. Die Patienten aus der Kontrollgruppe zeigten nach 4 Monaten keine Veränderung in ihrer Depressionsneigung; darüber hinaus stieg die Ängstlichkeit tendenziell an. Die Zufriedenheit in Ehe und Partnerschaft nahm in der Gruppe der Patienten, 
die an der Therapie teilnahmen eher zu. Hingegen konnte hier bei den Patienten der Kontrollgruppe keine Veränderung beobachtet werden.

Insgesamt wirkte sich die Teilnahme der PD-Patienten an der neuropsychologisch-verhaltenstherapeutisch orientierten Therapie positiv auf ihre kognitiven Leistungen und emotionale Befindlichkeit aus. Die Mehrzahl der geprüften kognitiven Parameter verbesserte sich tendenziell. Insbesondere die Aufmerksamkeit und die Leistungsfähigkeit des non-verbalen Gedächtnisses konnten signifikant gesteigert werden.

Demgegenüber musste für die PD-Patienten der Kontrollgruppe in einigen Parametern eine Stagnation, bei zahlreichen anderen eine signifikante Verschlechterung festgestellt werden. Auch die Erfassung der psychischen Aspekte ergab eine tendenzielle Verschlechterung in dieser Gruppe.

Die in der aktuellen Studie vorgestellte neue Kombinationstherapie gibt den Betroffenen ein stärkeres Gefühl der Kontrolle über ihre geistige Leistungsfähigkeit und kann somit sekundär die Lebensqualität verbessern.

Aufgrund der geringen Zahl der Versuchspersonen sind die Daten trotz der zunächst ermutigenden Ergebnisse unter Vorbehalt zu sehen. Die vorgestellte Therapie sollte zur weiteren Datenerhebung fortgeführt und später als Therapiemodul den Therapieblöcken Medikation und Physiotherapie hinzugefügt werden, da entsprechende kognitive Vorraussetzungen für den Erhalt bzw. für eine Verbesserung beeinträchtigter motorischer Fähigkeiten die Grundlage bilden.

Das Projekt wurde unterstützt durch die Deutsche Parkinson Vereinigung e.V. 


\section{Literatur}

Aarsland, D., Tandberg, E., Larsen, J. \& Cummings, J. (1996). Frequency of dementia in Parkinson's disease. Archives of Neurology 5: 538-542.

Aarsland, D., Ballard, C., McKeith, I., Perry, R.H. \& Larsen, J.P. (2001). Comparison of extrapyramidal signs in dementia with Lewy bodies and Parkinson's disease. Journal of Neuropsychiatry and Clinical Neuroscience 13: 374-379.

Alexander, M.P. \& Freedman, M. (1984). Amnesia after anterior communicating artery aneurysm rupture. Neurology 34: 752-757.

Alexander, G., DeLong, M.R. \& Strick, P.L. (1986). Parallel organization of functionally segregated circuits linking basal ganglia and cortex. Annual Review of Neuroscience 9: 357-381.

American Psychiatric Association (1994). Diagnostic and statistical manual of mental disorders ( $4^{\text {th }}$ ed). Washington, DC: American Psychiatric Press.

Anderson, S., Damasio, H., Jones, D. \& Tranel, D. (1991). Wisconsin Card Sorting Test performance as a measure of frontal lobe damage. Journal of Clinical and Experimental Neuropsychology 13: 909-922.

Ashby, F.G., Noble, S., Filoteo, J.V. \& Waldron, E.M. (2003). Category learning deficits in Parkinson's disease. Neuropsychology 17: 115-124.

Atkinson, R.C. \& Shiffrin, R.M. (1968). Human memory: A proposed system and its control processes. In K.W. Spence \& J.T. Spence (Eds.), The psychology of learning and motivation (pp. 92-122). New York: Academic Press.

Baddeley, A.D. (1986). Working memory. Oxford: Oxford University Press.

Baddeley, A.D. (1990). Human memory, theory and practice. Hillsdale, N.J: Lawrence Erlbaum Associates.

Beck, A.T. \& Steer, R.A. (1987). Beck Depression Inventory - Manual. San Antonio: The Psychological Association.

Beck, A.T., Steer, R.A. \& Garbin, M.G. (1988). Psychometric properties of the Beck Depression Inventory: Twenty-five years of evaluation. Clinical Psychology Review 8: 77-100.

Bernstein, D.A. \& Borkovec, T.D. (1975). Entspannungstraining. München: Pfeiffer.

Birbaumer, N. \& Schmidt, R.S. (1996). Biopsychologie. Berlin: Springer.

Bloxham, C.A., Dick, D.J. \& More, M. (1987). Reaction times and attention in Parkinson's disease. Journal of Neurology, Neurosurgery and Psychiatry 50: 1178-1183.

Bondi, M.W., Kaszniak, A.W., Bayles, K.A. \& Vance, K.T. (1993). Contributions of frontal system dysfunction to memory and perceptual abilities in Parkinson's disease. Neuropsychology 7: 89-102.

Brickenkamp, R. (1997). Handbuch psychologischer und pädagogischer Tests. Göttingen: Hogrefe. 
Brodmann, K. (1912). Ergebnisse über die vergleichende histologische Lokalisation der Großhirnrinde mit besonderer Berücksichtigung des Stirnhirns. Anatomischer Anzeiger 41: 157-216.

Brown, R.G. \& Marsden, C.D. (1986). Visuospatial function in Parkinson's disease. Brain 109: 987-1002.

Brown, R.G. \& Marsden, C.D. (1987). Neuropsychology and cognitive function in Parkinson's disease; an overview. In C.D. Marsden \& S. Fahn (Eds.), Movement disorders II (pp. 143-264). London: Butterworth.

Brown, R.G. \& Marsden, C.D. (1988). Internal versus external cues and the control of attention in Parkinson's disease. Brain 111: 323-345.

Brown, R.G. \& Marsden, C.D. (1990). Cognitive function in Parkinson's disease: From description to theory. Trends in Neuroscience 13: 21-29.

Brown, A. \& Gershon, S. (1993). Dopamine and depression. Journal of Neural Transmission 91: 75-109.

Bublak, P., Mueller U., Groen, G. Reuter, M. \& Cramon, D.Y. von (2002). Manipulation of working memory information is impaired in Parkinson's disease and related to working memory capacity. Neuropsychology 16: 577 590.

Bullinger, M., Kirchberger, I. \& Ware, J. (1995). Der deutsche SF-36 Health Survey. Übersetzung und psychometrische Testung eines krankheitsübergreifenden Instruments zur Erfassung der gesundheitsbezogenen Lebensqualität. Zeitschrift für Gesundheitswissenschaften 3: 21-36.

Bullinger, M. \& Kirchberger, I. (1998). SF-36 Fragebogen zum Gesundheitszustand, Handanweisung. Göttingen: Hogrefe.

Calabrese, P. (1997). Klinisch-neuropsychologische Gedächtnisdiagnostik. Grundlagen und Verfahren. In H.J. Markowitsch (Hrsg.), Enzyklopädie der Psychologie. Biologische Psychologie. Band 2 (S. 1051-1113). Klinische Neuropsychologie. Göttingen: Hogrefe.

Calabrese, P., Markowitsch, H.J. \& Tulving, E. (1998). Where in the cortex does cognition take place? In A. Neugebauer (Ed.), Macromolecular interplay in brain associative mechanisms (pp. 104-110). Singapore: World Scientific.

Canavan, A.G. (1983). Style-maze performance in patients with frontal-lobe lesions: Effects of signal valency and relationship to verbal and spatial abilities. Neuropsychologia 2: 375-382.

Canavan, A., Passingham, R., Mardsen, C. \& Quinn, N. (1989). Sequencing ability in Parkinsonians, patients with frontal lobe lesions and patients who have been undergoing unilateral temporal lobectomies. Neuropsychologia 27: 787-798.

Cicerone, K. \& Lazar, R. (1983). Effects of frontal lobe lesions on hypotheses sampling during concept formation. Neuropsychologia 21: 513-524.

Cohen, N.J. \& Squire, L.R. (1980). Preserved learning and retention of a patternanalyzing skill in amnesia: Dissociation of knowing how and knowing that. Science 210: 207-210. 
Cohen, N.J. (1984). Preserved learning capacity in amnesia. Evidence for multiple memory systems. In N. Butters \& L.R. Squire (Eds.), The neuropsychology of memory (pp. 83-103). New York: Guilford Press.

Cohen, N.J. (1985). Levels of analysis in memory research: The neuropsychological approach. In N.M. Weinberger, J.L. McGaugh \& G. Lynch (Eds.), Memory systems of the brain (pp. 351-380). New York: Guilford Press.

Coté, L. (1999). Depression: Impact and management by the patient and family. Neurology 52: 7-9.

Cramon, D.Y. von (1988). Problemlösen und Planung. In D.Y. von Cramon \& J. Zihl (Hrsg.) Neuropsychologische Rehabilitation. Grundlagen - Diagnostik Behandlungsverfahren (S. 248-263). Berlin: Springer.

Cramon, D.Y. von, Markowitsch, H.J. \& Schuri, U. (1993). The possible contribution of the septal region to memory. Neuropsychologia 3: 1159-1180.

Cramon, D.Y. von (1996). Neurobiologie des Arbeitsgedächtnisses. In H.J. Möller, F. Müller-Spahn \& G. Kurtz (Hrsg.) Aktuelle Perspektiven der Biologischen Psychiatrie (S. 1-11). Wien: Springer.

Cummings, J.L. (1992). Subcortical Dementia. New York: Oxford University Press.

Dalrymple-Alford, J.C., Kalders, A.S. \& Jones, R.D. (1994). A central executive deficit in patients with Parkinsons's disease. Journal of Neurology, Neurosurgery and Psychiatry 57: 360-367.

Danek, A. (2002). Neuropsychologie. In H. Förstl (Hrsg.), Frontalhirn Funktionen und Erkrankungen (S. 51-88). Berlin: Springer-Verlag.

DeLong, M.R. \& Georgopoulos, A.P. (1981). Motor functions of the basal ganglia. In J.M. Brookhart \& E.V. Mountcaste (Eds.), Handbook of Physiology (pp. 1017-1061). Bethesda: American Physiological Society.

Dörner, D. (1977). Problemlösen als Informationsverarbeitung. Stuttgart: Kohlhammer

Drewe, E.A. (1974). The effect of type and area of brain lesion on Wisconsin Card Sorting Test performance. Cortex 10: 159-170.

Dubois,B., Pillon, B., Sternic, N., Lhermitte, F. \& Agid, Y. (1990). Age-induced cognitive disturbance in Parkinson's disease. Journal of Neurology 40: 38-41.

Dubois, B. \& Pillon, B. (1997). Cognitive deficits in Parkinson's disease. Journal of Neurology 244: 2-8.

Duncan, J. (1986). Disorganization of behavior after frontal lobe damage. Cognitive Neuropsychology 3: 271-290.

Ellis, A. (1962). Reason and emotion in psychotherapy. New York: Lyle Stuart.

Ellis, A. (1977). Die Rational-Emotive Therapie. München: Pfeiffer.

Ellis, A. (1979). The basic clinical theory of rational emotive therapy. In A. Ellis \& M.M. Whitelay (Eds.), Theoretical and empirical foundations of rationalemotive therapy (pp. 33-60). Monterey, CA: Books Cole. 
Ellis, A. \& Hoellen, B. (1997). Die Rational-Emotive Verhaltenstherapie: Reflexionen und Neubestimmungen. München: Pfeiffer.

Eslinger, P. \& Grattman, L. (1993). Frontal lobe and frontal-striatial substrates for different forms of human cognitive flexibility. Neuropsychologia 31: 17-28.

Fahrenberg, J.,Hampel, R. \& Selg, H. (1985). Die revidierte Form des Freiburger Persönlichkeitsinventars FPI-R. Diagnostica 31: 1-21.

Fahrenberg, J., Hampel, R. \& Selg, H. (1994). Das Freiburger Persönlichkeitsinventar (FPI) - Revidierte Fassung. Göttingen: Hogrefe.

Fahrenberg, J., Myrtek, M., Schumacher, J. \& Brähler, E. (2000). Fragebogen zur Lebenszufriedenheit (FLZ). Göttingen: Hogrefe.

Fletcher, P.C., Frith, C.D. \& Rugg, MD. (1997). The functional neuroanatomy of episodic memory. Trends in Neurosciences 20: 213-218.

Flowers, K.A., Pearce, I. \& Pearce, J.M.S. (1984). Recognition memory in Parkinson's disease. Journal of Neurology, Neurosurgery and Psychiatry 4: 1174 - 1181

Freedman, P., Bleiberg, J. \& Freedland, K. (1987). Anticipatory behavior deficits in closed head injury. Journal of Neurology, Neurosurgery and Psychiatry 50: 398-401.

Freedman, M. (1990b). Object alternation and orbitofrontal system dysfunction in Alzheimer's and Parkinson's disease. Brain and Cognition 14: 134-143.

Frith, C., Friston, K., Liddle, P. \& Frackowiak, R. (1991). A PET study of word finding. Neuropsychologia 29: 1137-1148.

Funke, J. \& Grube-Unglaub, S. (1993). Skriptgeleitete Diagnostik von Planungskompetenz in neuropsychologischem Kontext: Erste Hinweise auf die Brauchbarkeit des Skript-Monitoring-Test (SMT). Zeitschrift für Neuropsychologie 4: 75-91.

Fuster, J.Q. (1997). The prefrontal cortex. Anatomy, physiology and neuropsychology. Philadelphia, PA.: Lippincott-Raven.

Gamsu, S. V. (1986). Confrontation naming in Parkinsonian patients: postoperative anomia revisited. Neuropsychologia 24: 727-729.

Gauntlett-Gilbert, J. \& Brown, V.J. (1998). Reaction time deficits and Parkinson's disease. Neuroscience and Biobehavior Review 22: 865-881.

Gediga, G. \& Schöttke, H. (1986). Die Wirkung der Übung auf den Problemlöseverlauf. Zeitschrift für Experimentelle und Angewandte Psychologie 33: 375-385.

Gediga, G. \& Schöttke, H. (1994). Turm von Hanoi (TvH). Göttingen: Hogrefe.

Gibb, W. \& Lees, A.J. (1988). A comparison of clinical and pathological features of young- and old-onset Parkinson's disease. Neurology 38: 14021406.

Glosser, G., Clark, C. \& Freundlich, B. (1995). A controlled investigation of current and premorbid personality: characteristics of Parkinson's disease patients. Movement Disorders 10: 201-206.

Gluck, M.A. \& Myers, C.E. (1997). Psychobiological models of hippocampal function in learning and memory. Annual Review of Psychology 48: 481-514. 
Goldberg, E. \& Bilder, R.M. (1987). The frontal lobes and hierarchical organization of cognitive control. In $\mathrm{E}$. Perecman (Ed.), The frontal lobes revisited (pp.159-187). New York: IRBN Press.

Härting, C., Markowitsch, H.J., Neufeld, H., Calabrese, P., Deisinger, K. \& Kessler, J. (2000). Wechsler Gedächtnis Test - Revidierte Fassung (WMS-R). Bern: Huber.

Halbig, T., Gruber, C.A., Doreen, M., Kopp, U., Scherer, P., Schneider, G.H., Trottenberg, T., Arnold, G. \& Kupsch, A. (2004). Subthalamic stimulation differentially modulates declarative and nondeclarative memory. Neuroreport 15: 539-543.

Hamilton, M. (1960). A rating scale for depression. Journal of Neurology Neurosurgery and Psychiatry 23: 56-62.

Hampel, R. \& Klinkhammer, F. (1978). Verfälschungstendenzen beim Freiburger Persönlichkeitsinventar in Bewerbungssituationen. Psychologie und Praxis 22: 58-69.

Hautzinger, M. \& Bailer, M. (1991). Allgemeine Depressions Skala. Weinheim: Beltz Test.

Hautzinger, M., Bailer, M., Worall, H. \& Keller, F. (1995). Beck-DepressionsInventar (BDI)-Testhandbuch. Bern: Hans Huber.

Hautzinger, M. \& Bailer, M. (1996). Inventar Depressiver Symptome. Weinheim: Beltz Test.

Heberlein, I., Ludin, H.P., Scholz, J. \& Vieregge, P. (1998). Personality, depression, and premorbid lifestyele in twin pairs dirscordant for Parkinsons's disease. Journal of Neurology, Neurosurgery and Psychiatry 64: 262-266.

Hobson, P. \& Meara, J. (1999). The detection of dementia and cognitive impairment in a community population of elderly people with Parkinson's disease by use of the CAMCOG neuropsychological test. Age and Aging 28: 39-43.

Hoehn, M.M. \& Yahr, M.D. (1967). Parkinsonism. Onset, progression and mortality Neurology 17: 427-442.

Hollmann, H. (1988). Das Freiburger Persönlichkeitsinventar. Diagnostica 34: 277-285.

Horne, D.J. (1971). Performance on delayed response tasks in patients with parkinsonism. Journal of Neurology, Neurosurgery and Psychiatry 34: 192194.

Howes, D. \& Boller, F. (1975). Simple reaction time: Evidence for focal impairments from lesions of the right hemisphere. Brain 98: 317332.

Hurwitz, T.A. \& Calne, S.M. (2001). Depression, anxiety and psychosis in Parkinson's disease. British Columbia Medical Journal 43: 214-218.

Jaeger, R. S. (1985). Testinformationen. Das Freiburger Persönlichkeitsinventar (FPI). Diagnostica 31: 246-250.

Jahanshahi, M., Brown, M.G. \& Marsden, C.D. (1992). Simple and choice reaction time and the use of advance information for motor preparation in Parkinson's disease. Brain 115: 539-564. 
Janke, W., Erdmann, G. \& Boucsein, W. (1978). Der Stressverarbeitungsfragebogen. Ärztliche Praxis 30: 1208-1210.

Janke, W., Erdmann, G. \& Kallus, W. (1985). Der Streßverarbeitungsfragebogen (SVF). Göttingen: Hogrefe.

Janke, W. \& Erdmann, G. (1997). Der Streßverarbeitungsfragebogen (SVF 120). Kurzbeschreibung und grundlegende Kennwerte. Göttingen: Hogrefe.

Kanfer, F.H. (1970). Self-regulation and self-control. In H. Zeir (Ed.), The psychology of the 20th century. From classical conditioning to behavioral therapy (pp. 793-827). Zürich: Kindler.

Kanfer, F.H., Reinecker, H. \& Schmelzer, D. (1996). SelbstmanagementTherapie. Berlin: Springer.

Karnath, H.O. (1991). Zur Funktion des präfrontalen Cortex bei mentalen Planungsprozessen. Zeitschrift für Neuropsychologie 2: 14-28.

Karnath, H.O., Wallesch, C.W. \& Zimmermann, P. (1991). Mental planning and anticipatory processes with acute and chronic frontal lobe lesions: A comparison of maze performance in routine and non-routine situations. Neuropsychologia 29: 271-290.

Kendall, P.C., Finch, A.J., Auerbach, S.M., Hooke, J.F. \& Mikulka, P.J. (1976). The State-Trait Anxiety Inventory: A systematic evaluation. Journal of Consulting and Clinical Psychology 44: 406-412.

Keßler, B. H. \& Hoellen, B. (1982). Rational-Emotive Therapie in der Klinischen Praxis. Weinheim:Beltz.

Keßler, B. H. (1994). Rational-Emotive Therapie. In R. Asanger \& G. Wenniger, (Hrsg.), Handwörterbuch Psychologie (S. 1105-1126). München: PVU.

König, F. \& Schmidt, J. U. (1982). Skalen- und Strukturanalyse des Freiburger Persönlichkeitsinventars (FPI). Diagnostica 28: 33-48.

Konow, A. \& Pribram, K. (1970). Error recognition and utilization produced by injury to the frontal cortex in man. Neuropsychologia 8: 489-491.

Kühl, K.P. \& Baltes, M.M. (1989). Dementielle Erkrankungen im Alter: Früherkennung mit Hilfe des 'Testing-the-Limits'-Ansatzes. Zeitschrift für Gerontopsychologie und -psychiatrie 2: 289-293.

Kuzis, G., Sabe, L. \& Tiberti, C. (1997). Cognitive functions in major depression and Parkinson's disease. Archives of Neurology 54: 982-986.

Ladavas, E. (1987). Is hemispational deficit produced by right parietal lobe damage associated with retinal or gravitational coordinates? Brain 110: $167-180$.

Lazarus, R.S. (1966). Psychological Stress and the Coping Process. New York: McGraw Hill.

Lees, A.J. \& Smith, E. (1983). Cognitive deficits in the early stages of Parkisnon's disease. Brain 106: 257-70.

Lehrl, S. (1989). Mehrfachwahl-Wortschatz-Intelligenztest MWT-B. Erlangen: Perimed.

Lehrl, S. \& Fischer, B. (1990). A basic information psychological parameter (BIP) for the reconstruction of intelligence. European Journal of Personality 4: 259-286. 
Lehrl, S., Gallwitz, A., Blaha, L. \& Fischer, B. (1991). Theorie und Messung der biologischen Intelligenz mit dem Kurztest KAl. Die allgemeinen Basisgrößen der Informationsverarbeitung. Ebersberg: Vless.

Lehrl, S. \& Fischer, B. (1998). The basic parameters of human information processing: Their role in the determination of intelligence. Personality and Individual Differences 9: 883-896.

Lein, B.E., Liabre, M.M. \& Reisman, S. (1991). Visuospatial impairment in Parkinson's disease. Neurology 41: 365-369.

Leplow, B., Bamberger, D., Moebius, T. \& Ferstl, B. (1993). Verhaltenstherapeutische Gruppenprogramme bei Parkinson-Patienten. TW Neurologie und Psychiatrie 7: 59-68.

Lichter, D.G. (2001). Movement disorders and frontal-subcortical circuits. In D.G. Lichter \& J.L. Cummings (Eds.), Frontal-subcortical circuits in psychiatric and neurological disorders (pp. 260-313). New York: Guilford.

Linden, M. \& Hautzinger M. (1996). Verhaltenstherapie. Praxisbezogene Darstellung verhaltenstherapeutischer Techniken und Einzelverfahren. Berlin: Springer.

Lukesch, H. (1974). Testkriterien des Depressionsinventars von A.T. Beck. Psychologie \& Praxis 18: 60-78.

Luria, A.R. (1973). The working brain. Harmondsworth: Penguin Books.

Mahurin, R.K., Feher, E.P., Nance, M.L., Levy, J.K. \& Pirozzolo, F.J. (1993). Cognition in Parkinson's disease and related disorders. In R.W. Parks, R.F. Zec, \& R.S. Wilson (Eds.), Neuropsychology of Alzheimer's disease and other dementias (pp. 308-349). New York: Oxford University Press.

Marker, K.R. (1996). Handbuch zum Programmpaket Cogpack. Version 4.5. Ladenburg: Marker Software.

Markowitsch, H.J. (1988). Anatomical and functional organization of the primate prefrontal cortical system In H.D. Steklis \& J. Erwin (Eds.), Comparative primate biology. Vol. IV. Neurosciences (pp. 99-153). New York: Alan R. Liss.

Markowitsch, H.J. (1995). Which brain regions are critically involved in the retrieval of old episodic memory? Brain Research Review 21: 117-127.

Markowitsch, H.J. (1999). Gedächtnisstörungen. Stuttgart: Kohlhammer.

Markowitsch, H.J. (2002). Dem Gedächtnis auf der Spur. Darmstadt: Wissenschaftliche Buchgesellschaft.

Martinez-Martin,P., Benito-Leon,J., Alonso, F., Catalan, M.J., Pondal, M., Zamarbide, I., Tobias, A. \& Pedro, J. de (2005). Quality of life of caregivers in Parkinson's disease. Quality of Life Research 14: 463-72.

Matthes-von Cramon, G. \& Cramon, D.Y. von (2000): Störungen exekutiver Funktionen. In W. Sturm, M. Herrmann \& C.W. Wallesch (Hrsg.), Lehrbuch der Klinischen Neuropsychologie (S. 392-410). Lisse, NL: Swets \& Zeitlinger.

Mayeux, R., Stern, Y. \& Cote, L. (1984). Altered serotonin metabolism in depressed patients with Parkinson's disease. Neurology 34: 642-646.

Mega, M.S. \& Cummings, J.L. (1994). Frontal-subcortical circuits and neuropsychiatric disorders. Journal of Neuropsychiatry and Clinical Neuroscience 6: 358-370.

Meichenbaum, D. (1977). Cognitive-behavior modification. New York: Plenum Press. 
Meichenbaum, D. (1995). Kognitive Verhaltensmodifikation. Weinheim: Beltz.

Mesulam, M.M. (1985). Attention, confusional states, and neglect. In M.M. Mesulam (Ed.), Principles of Behavioral Neurology (pp. 124-168). Philadelphia: Davis.

Miller, G.A. (1956). The magical number seven plus minus two. Some limits on our capacity for processing information. Psychology Review 63: 244-257.

Millner, B. (1964). Some effects of frontal lobectomy in man. In J.M. Warren \& K. Akert (Eds.), The Frontal Granular Cortex and Behavior (pp. 313-334). New York: MCGraw-Hill.

Mueller, U., Wachter, T., Barthel, H., Reuter, M. \& Cramon, D.Y. von (2000). Striatal beta-CIT SPECT and prefrontal cognitve functions in Parkinson's disease. Journal of Neural Transmission 107: 303-319.

Neher, K.D. (1990). Morbus Parkinson. Stuttgart: Hippokrates.

Nelson, H.E. (1976). A modified card sorting test sensitive to frontal lobe defects. Cortex 12: 313-324.

Norman, D.A. \& Shallice, T. (1986). Attention to action. Willed and automatic control of behaviour. In R.J. Davidson, G.E. Schwartz \& D. Shapiro (Eds.), Consciousness and Self-Regulation (pp.1-18). New York: Plenum Press.

Normann, S., Troster, A.L., Fields, J.A. \& Brooks, R. (2002). Effects of depression and Parkinson's disease on cognitive functioning. Journal of Neuropsychology and Clinical Neuroscience 14: 31-36.

Owen, A.M. (1977). Cognitive planning in humans: Neuropsychological, neuroantatomical and neuropharmacological perspectives. Progress in Neurobiology 53: 431-450.

Owen, A.M., James, M., Leigh, P.N., Summers, B.A., Marsden, C.D. \& Quinn, N.P. (1992). Frontostriatal cognitive deficits at different stages of Parkinson's disease. Brain 115: 1727-1751.

Owen, A.M., Iddon, J.L. \& Hodges, J.R. (1997). Spatial and non-spatial working memory at different stages of Parkinson's disease. Neuropsychologia 35: 519-532.

Parkinson, J.R. (1817). An Essay on the Shaking Palsy. London: Sherwood, Neely and Jones.

Pfingsten, U. (1996). Training sozialer Kompetenz. In J. Margraf (Hrsg.), Lehrbuch der Verhaltenstherapie (S. 473-481). Berlin: Springer.

Pfingstmann, G. \& Baumann, U. (1986). Zur Validierung der FPI-R-Version anhand von Klienten einer Studentenberatungsstelle. Zeitschrift für Differentielle und Diagnostische Psychologie 7: 243-247.

Posner, M.I. \& Boies, S.W. (1971). Components of attention. Psychological Review 78: 391-408.

Posner, M.I. \& Rafal, R.D. (1987). Cognitive theories of attention and the rehabilitation of attentional deficits. In R.J. Meier, A.C. Benton \& L. Diller (Eds.), Neuropsychological Rehabilitation (pp. 182-201). Edingburgh: Churchill Livingstone.

Posner, M.I. \& Petersen, S.E. (1990). The attention system of the human brain. Annual Review of Neuroscience 13:182-196.

Ransmayr, G. (1987). Visuoperception and visuospatial and visuorotational performance in Parkinson's disease. Journal of Neurology 23: 99-101. 
Reitan, R.M. (1958). Validity of the trailmaking test as an indication of organic brain damage. Perceptual and Motor Skills 8: 271-276.

Richards, M., Cote, I.J. \& Stern, Y. (1993). Executive function in Parkinson's disease: Set-shifting or set-maintenance? Journal of Clinical and Experimental Neuropsychology 15: 266-279.

Robinson, A.L. Heaton, R.K., Lehman, R.A. \& Stilson, D.W. (1980). The utility of the Wisconsin Card Sorting Test in detecting and localizing frontal lobe lesions. Journal of Consulting and Clinical Psychology 48: 605-664.

Röhrenbach, C. \& Markowitsch, H.J. (1997). Störungen im Bereich exekutiver und überwachender Funktionen - der Präfrontalbereich. In H.J. Markowitsch (Hrsg.), Klinische Neuropsychologie. Enzyklopädie der Psychologie (S. 329493). Göttingen: Hogrefe.

Roether, D. (1985). Tempoleistung und Merkfähigkeit Erwachsener (TME). Berlin: Psychodiagnostisches Zentrum.

Rogers, D., Lees, A.J., Smith, E., Trimble, M. \& Stern, G. (1987). Bradyphrenia in Parkinson's disease and psychomotor retardation in depressive illness. An experimental study. Brain 110: 761-776.

Saint-Cyr, J.A. (2003). Neuropsychology for Movement Disorder Neurosurgery. The Canadian Journal of Neurological Sciences 1: 83-93.

Salmon, D.P., Heindel, W.C. \& Hamilton, J.M. (2001). Cognitive abilities mediated by frontal-subcortical circuits. In D.G. Lichter \& J.L. Cummings (Eds.), Frontal-subcortical cicruits in psychiatric and neurological disorders (pp.114-150). New York: Guilford.

Santamaria, J., Tolosa, E. \& Valles, A. (1988). Parkinson's disease with depression: a possible subgroup of idiopathic parkinsonism. Neurology 36: 1130-1133.

Schaffner, P. \& Spielberger, C.D. (1981). Das State-Trait-Angstinventar. Theoretische Grundlagen und Handanweisung. Weinheim: Beltz Test.

Schmidt, J. U. \& König, F. (1986). Untersuchungen zur Validität der revidierten Form des Freiburger Persönlichkeitsinventars FPI-R. Diagnostica, 3: 197-208.

Schöttke, H. (1990). Zur diagnostischen Erfassung von Planungs- und Problemlösestörungen nach einer traumatischen Hirnschädigung. In D. Frey (Hrsg.), Bericht über den 37. Kongress der DGP in Kiel 1990 (S.129). Göttingen: Hogrefe.

Schöttke, H. (1993). Zur Störung der Intentionalität bei schizophrenen und hirngeschädigten Patienten: Nosologische Spezifität und Validität. Zeitschrift für Medizinische Psychologie 2: 17-26.

Scholz, E. \& Oertel, W.H. (1993). Parkinson-Syndrome. In T. Brandt, J. Dichgans \& H. Diener (Hrsg.), Therapie und Verlauf neurologischer Erkrankungen (S. 927-968). Stuttgart: Kohlhammer.

Schwenkmetzger, P. (1987). Freiburger Persönlichkeitsinventar (FPI). Revidierte Fassung FPI-R. Zeitschrift für Differentielle und Diagnostische Psychologie 8: 152-153.

Secker, D.L. \& Brown, R.G. (2005). Cognitive behavioural therapy (CBT) for carers of patients with Parkinson's disease: a preliminary randomised controlled trial. Journal of Neurology, Neurosurgery and Psychiatry 76: 491497. 
Shallice, T. (1982). Specific impairments of planning. In D. Broadbent \& F. Weiskrantz (Eds.), The neuropsychology of cognitive function (pp. 254-277). London: Royal Society.

Shallice, T. (1988). From Neuropsychology to Mental Structure. Cambridge: England Cambridge University Press.

Shimamura, A.P. (1995). Memory and frontal lobe function. In M.S. Gazzaniga (Ed.), The cognitive neurosciences (pp. 803-813). Cambridge: MIT Press.

Soukup, V.M. \& Adams, R.L. (1996). Parkinson's disease. In R.L. Adams, O.A. Parsons, J.L. Culbertson \& S.J. Nixon (Eds.), Neuropsychology for clinical practice: Etiology, assessment, and treatment of common neurological disorders (pp. 243-267). Washington, DC: American Psychological Association.

Spielberger, C.D., Gorsuch, R.L. \& Lushene, R.E. (1970). Manual for the StateTrait Anxiety Inventory. Pablo Alto, Ca.: Consulting Psychologists Press.

Spielberger, C.D. (1971). Trait-state anxiety and motor behavior. Journal of Behavior 3: 269-279.

Spielberger, C.D. (1980). Streß und Angst. Weinheim: Beltz.

Squire, L.R. \& Zola-Morgan, S. (1991). The medial temporal lobe memory System. Science 253: 1380-1386.

Stachowiak, F.J., De Bleser, R., Deloche, G., Kaschel, R., Kremin, H., North, P., Pizzamiglio, L., Robertson, I. \& Wilson, B. (1993). Developments in the Assessment and Rehabilitation of Brain-Damaged Patients. Perspectives from a European Concerted Action. Tübingen: G. Narr Verlag.

Starkstein, S.E., Berthier, M.L. \& Bolduc, P.L. (1989). Depression in patients with early versus late onset of Parkinson's disease. Neurology 37: 1441-1445.

Starkstein, S.E., Bolduc, P.L., Mayberg, H.S., Preziosi, T.J. \& Robinson, R.G. (1990). Cognitive impairments and depression in Parkinson's disease: a follow up study. Journal of Neurology, Neurosurgery and Psychiatry 53: 597602.

Steinmeyer, E.M. (1993). Zur klinischen Validität des Beck-Depressioninventars. Nervenarzt 64: 717-726.

Sturm, W., De Simone, A., Krause, B., Specht, K., Hesselmann, V., Radermacher, I., Herzog, H., Tellmann, L., Müller-Gärtner, W.W. \& Wilmes, K. (1999). Functional anatomy of intrinsic alertness: Evidence for a frontoparietal-thalamic-brainstem network in the right hemisphrere. Neuropsychologia 37: 797-805.

Stuss, D. \& Benson, D. (1984). Neuropsychological studies of the frontal lobes. Psychological Review 95: 3-28.

Swerdlow, N.R. \& Koob, G.F. (1987). Dopamine, schizophrenia, mania, and depression: Toward a unified hypothesis in cortico-striato-pallido-thalamic function. Behavioral and Brain Sciences 10: 197-245.

Talland, G.A. \& Schwab, R.S. (1964). Performance with multiple sets in Parkinson's disease. Neuropsychologia 2: 45-53.

Tandberg, E. Larsen, J.P. \& Aarsland, D. (1996). The occurrence of depression in Parkinson's disease: a community-based study. Archives of Neurology 53: 75-179. 
Taylor, A.E., Saint-Cyr, J.A. \& Lang, A.E. (1986). Frontal lobe dysfunction in Parkinson's disease. Brain 109: 845-883.

Taylor, A.E., Saint-Cyr, J.A. \& Lang, A.E. (1987). Parkinson's disease. Cognitive changes in relation to treatment response. Brain 110: 35-51.

Taylor, A.E. \& Saint-Cyr, J.A. (1990). Depression in Parkinson's disease: reconciling physiological and psychological perspectives. Journal of Neuropsychiatry and Clinical Neuroscience 2: 92-98.

Taylor, A.E. \& Saint-Cyr, J.A. (1995). The neuropsychology of Parkinson's disease. Brain 28: 281-296.

Troster, A.I., Stalp, L.D, \& Paolo, A.M. (1995). Neuropsychological impairment in Parkinson's disease with and without depression. Archives of Neurology 52: 1164-11169.

Tulving, E. (1983). Elements of episodic memory. Oxford: Oxford University Press.

Tulving, E. \& Schachter, D.L. (1990). Priming and human memory systems. Science 247: 301-306.

Vagg, P.R., Spielberger, C.D. \& O`Hearn, T.P. (1980). Is the State-Trait Anxiety Inventory multidimensional? Journal of Personality and Individual Difference 22: 28-33.

Vilkki, J. \& Holst, P. (1989). Deficient programming in spatial learning after frontal lobe damage. Neuropsychologia 27: 971-976.

Wallesch, C.W. \& Herrmann, M. (2000). Klinische Neurologie. In W. Sturm, M. Herrmann \& C.W. Wallesch (Hrsg.), Lehrbuch der klinischen Neuropsychologie (S. 96-125). Lisse: Swets \& Zeitlinger Publishers.

Wallesch, C.W. (2002). Therapie frontalexekutiver Störungen: Grundsätzliche Überlegungen. In $\mathrm{H}$. Förstl (Hrsg.), Frontalhirn - Funktionen und Erkrankungen (S. 329-345). Heidelberg: Springer.

Walsh, K. (1987). Neuropsychology. A clinical approach. Edingburgh: Churchill Livingstone.

Wasserstein, J., Borod, J.C., Bodis-Wolner, I., Goldstein, Y. \& Yahr, M. (1987). Visual-perceptual deficits in Parkinson's disease. Journal of Clinical and Experimental Neuropsychology 9: 65-73.

Wechsler, D. (1987). WMS-R: Wechsler Memory Scale Revised (Manual). San Antonio: The Psychological Corporation.

Weingartner, H., Burns, S., Diebel, R. \& Le Witt, P.A. (1984). Cognitive Impairment in Parkinson's disease. Psychiatry Research 11: 223-235.

Weintraub, D., Newberg, A.B., Cary, M.S., Siderow, A.D., Moberg, P.J., KleinerFisman, G., Duda, J.E., Stern, M.B., Mozley, D. \& Katz, I.R. (2005). Striatal dopamine transporter imaging correlates with anxiety and depression symptoms in Parkinson's disease. Journal of Nuclear Medicine 46: 227-32.

Weniger, G., Markowitsch, H.J. \& Irle, E., (1995). Anterograde and retrograde mnemonic deficits after unilateral damage of neostriatal, ventral striatal, and basal forebrain structures. Neurocase 1: 231-238.

Wickelgren, I. (1997). Getting a grasp on working memory. Science 175: 15801582.

Wilson, R.S., Kaszniak, A.W., Klawans, H.L. \& Garron, D.C. (1980). High speed memory scanning in Parkinsonism. Cortex 16: 67-72. 
Wolpe, A. (1982). Systematische Desensibilisierung. In R. Keeser (Hrsg.), Schmerz. Fortschritte der klinischen Psychologie, Bd. 27. München: Urban \& Schwarzenberg.

Woods, S.P. \& Troster, A.I. (2003). Prodromal frontal/executive dysfunction predicts incident dementia in Parkinson's disease. Journal of the International Neuropsychological Society 9: 17-24.

Zakzanis, K. \& Freedman, M. (1999). A neuropsychological comparison of demented and nondemented patients with Parkinson's disease. Applied Neuropsychology 6: 129-146.

Zerssen, D. von (1976). Beschwerdenliste. Weinheim: Beltz Test.

Zimmermann, P., Sprengelmeyer, R., Fimm, B. \& Wallesch, C.W. (1992). Cognitive slowing in decision tasks in early and advanced Parkinson's disease. Brain and Cognition 18: 60-69.

Zimmermann, P. \& Fimm, B. (1993). Testbatterie zur Erfassung von Aufmerksamkeitsstörungen. Version 1.02. Freiburg: Psytest.

Zimmermann, P. \& Fimm, B. (2002). A test battery for attentional performance. In M. Leclercq \& P. Zimmermann (Eds.), Applied Neuropsychology of Attention. Theory, Diagnosis and Rehabilitation (pp. 110-151). London: Psychology Press.

Zoccolotti, P., Matano, A., Deloche, G., Cantagallo, A., Passadori, A., Leclercq, M., Braga, L., Cremel, N., Pittau, P., Renom, M., Rousseaux, M., Truche, A., Fimm, B. \& Zimmermann, P. (2000). Patterns of attentional impairment following closed head injury: A collaborative European study. Cortex 36: 93107.

Zung, W.K. (1965). A self-rating depression scale. Archives of General Psychiatry 12: 63-70. 